\title{
Initial Value Problems for Creeping Flow of Maxwell Fluids
}

\author{
Toufik Laadj
}

\author{
Dissertation submitted to the Faculty of the \\ Virginia Polytechnic Institute and State University \\ in partial fulfillment of the requirements for the degree of
}

Doctor of Philosophy

in

Mathematics
Michael Renardy, Chair
Slimane Adjerid, Tao Lin,
Yuriko Renardy,

\author{
February 24, 2011 \\ Blacksburg, Virginia
}

Keywords: Quasistatic viscoelastic flow, nonlinear Maxwell fluid, unsteady flow, existence and uniqueness

Copyright@2011, Toufik Laadj 


\title{
Initial Value Problems for Creeping Flow of Maxwell Fluids
}

\author{
Toufik Laadj
}

\begin{abstract}
We consider the flow of nonlinear Maxwell fluids in the unsteady quasistatic case, where the effect of inertia is neglected. We study the well-posedness of the resulting PDE initialboundary value problem. This well-posedness depends on the unique solvability of an elliptic boundary value problem. We first present results for the 3D case, locally and globally in time, with sufficiently small initial data, and for a simple shear flow problem, locally in time with arbitrary initial data; after that we extend our results to some 3D flow problems, locally in time, with large initial data. Additionally, we present results for models of White-Metzner type in 3D flow, locally and globally in time, with sufficiently small initial data.

We solve our problem using an iteration between elliptic and hyperbolic linear subproblems. The limit of the iteration provides the solution of our original problem.
\end{abstract}




\section{Dedication}

I would like to dedicate this work to

my loving parents,

my sisters,

my brothers

and my future wife. 


\section{Acknowledgments}

I wish first to express my deepest gratitude to my advisor Professor Michael Renardy, for his guidance and help, and for sharing his knowledge. I am also very grateful to him for his patience, availability and prompt reply to my emails. It is not possible for me to express my gratitude to him in only a few lines.

I would like to express my deep gratitude to Professor Yuriko Renardy, Professor Slimane Adjerid, and Professor Tao Lin, to have honored me by being part of my committee. I would like to offer my utmost thanks to them for agreeing to serve on the committee.

Very special thanks and appreciation to Professor Mohamed Medjden, and Professor Slimane Adjerid, for their help in making me a Virginia Tech student (Hokie).

I also wish to thank all my professors and other persons having contributed closely or by far to the completion of this work.

Finally, I would like to thank my family and friends, especially my parents, my brother Zaid and my friend Naamane Bermitta, for their support throughout my studies. 


\section{Contents}

Contents $\quad$ v

$\begin{array}{ll}\text { List of Figures } & \text { ix }\end{array}$

List of Notations $\quad$ x

$\begin{array}{ll}\text { Introduction } & 1\end{array}$

1 Presentation of the problem $\quad 5$

1.1 Some basic concepts of fluid dynamics . . . . . . . . . . . . . 6

1.1.1 Representation of the motion .............. 6

1.1.2 Three-, two- and one-dimensional flows . . . . . . . . . . 6

1.1 .3 Velocity field ..................... 7

1.1.4 Steady and unsteady flow .................. 7

1.1.5 Material derivative and fluid acceleration . . . . . . . . . 8

1.2 Fundamental equations of fluid dynamics . . . . . . . . . . . . 8

1.2.1 Conservation of mass . . . . . . . . . . . . . 8 
1.2.2 Balance of momentum . . . . . . . . . . . . . . 9

1.2 .3 Constitutive equations . . . . . . . . . . . . . . 10

1.2.4 Newtonian and non-Newtonian fluids . . . . . . . . . . . . . 10

1.2.5 Boundary and initial conditions . . . . . . . . . . . . . . . 10

1.3 Viscoelastic fluid flow . . . . . . . . . . . . . . . . . . 11

1.3.1 Quasistatic or creeping viscoelastic flow . . . . . . . . . . . 11

1.3.2 Viscoelastic constitutive models . . . . . . . . . . . . . . . 11

1.3.3 Viscoelastic simple shear flow .................. 14

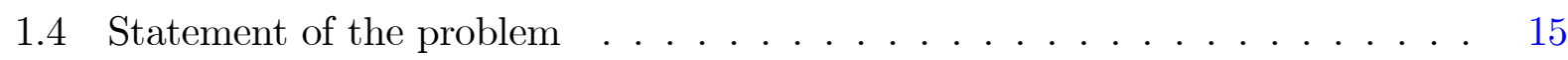

1.4.1 Physical description of the problem . . . . . . . . . . 15

1.4.2 Mathematical formulation ................. 16

2 Some basic mathematical concepts $\quad 18$

2.1 Notations and definitions . . . . . . . . . . . . . . . . 19

2.1 .1 Distributions . . . . . . . . . . . . . . . 19

2.1.2 Sobolev spaces . . . . . . . . . . . . . . 20

2.2 Basic results on Sobolev spaces . . . . . . . . . . . . . . . . 22

2.2.1 Approximation by smooth functions . . . . . . . . . . . . . 22

2.2.2 Trace and extension theorems . . . . . . . . . . . . . 22

2.2 .3 Divergence theorem . . . . . . . . . . . . . . 23

2.2.4 Sobolev embedding theorem . . . . . . . . . . . . . 24

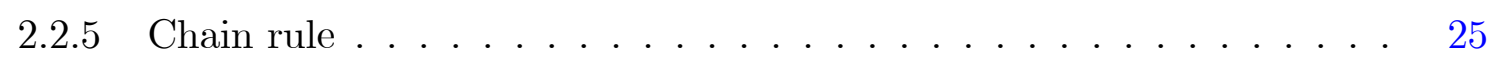

2.3 Implicit function theorem . . . . . . . . . . . . . . . 26 
3 Preliminary problems $\quad 28$

3.1 Stokes problem . . . . . . . . . . . . . . . . 28

3.2 Evolution problem . . . . . . . . . . . . . . . . . . 29

4 Three-dimensional flow with small initial data 38

4.1 Preliminaries and main result f . . . . . . . . . . . . . 39

4.2 Reduction to a sequence of Stokes and evolution problems . . . . . . . . . 40

4.3 Construction of the sequence $\left(V^{n}, q^{n}, p^{n}, \mathbf{T}^{n}\right) \ldots \ldots \ldots \ldots$

4.3 .1 Existence and uniqueness ..................... 43

4.3 .2 Convergence ........................ 46

5 Unsteady simple shear flow with arbitrary initial data $\quad 50$

5.1 Problem formulation . . . . . . . . . . . . . . 50

5.2 Reduction to iterative subproblems . . . . . . . . . . . 55

5.3 Existence and uniqueness . . . . . . . . . . . . . . 56

5.4 Convergence of the scheme . . . . . . . . . . . . . . 61

6 Extension to some three-dimensional flows with large initial data $\quad 64$

6.1 Assumptions on the initial data $\mathbf{T}_{0} \ldots \ldots \ldots \ldots$

6.1.1 Johnson-Segalman model with constant initial data $\mathbf{T}_{0} \ldots \ldots 6$

6.2 'Modified' Stokes problem . . . . . . . . . . . . . . . 69

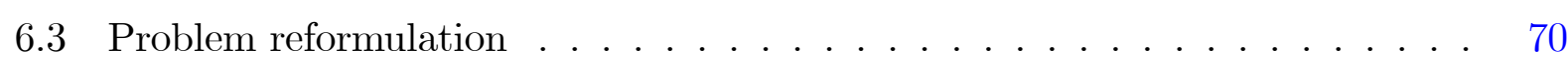

6.3.1 Reduction to iterative subproblems . . . . . . . . . . 72

6.4 Construction of the solution $\ldots \ldots \ldots \ldots \ldots$

6.4 .1 Existence .......................... 73 


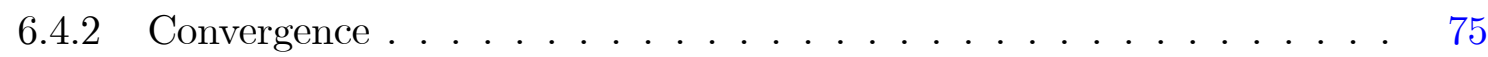

7 Models of White-Metzner type in 3D flow with sufficiently small initial $\begin{array}{ll}\text { data } & 78\end{array}$

7.1 Problem reformulation . . . . . . . . . . . . . . . 80

7.2 Assumptions on $\mu$ and $\lambda \ldots \ldots \ldots \ldots$. . . . . . . . . . . . . .

7.3 Transformation to iterative scheme . . . . . . . . . . . . . 82

7.4 Construction of the solution $\ldots \ldots \ldots \ldots$

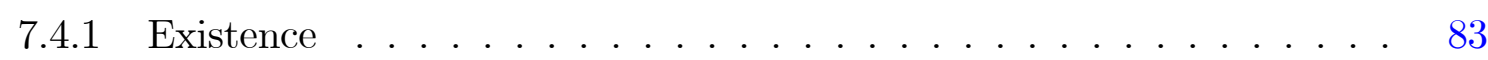

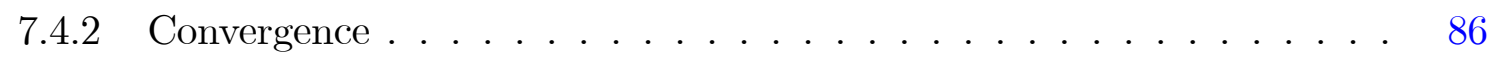

$\begin{array}{lr}\text { Conclusion and perspectives } & 89\end{array}$

$\begin{array}{ll}\text { Bibliography } & 90\end{array}$ 


\section{List of Figures}

1.1 Simple shear flow: A fluid placed between two parallel plates. . . . . . . . . 14

1.2 Fluid flow domain . . . . . . . . . . . . . . . . . . 15 


\section{List of Notations}

$\alpha \quad m$-dimensional multi-index defined as $\alpha=\left(\alpha_{1}, \alpha_{2}, \ldots, \alpha_{m}\right) \ldots \ldots \ldots \ldots \ldots \ldots \ldots \ldots \ldots$ page 19

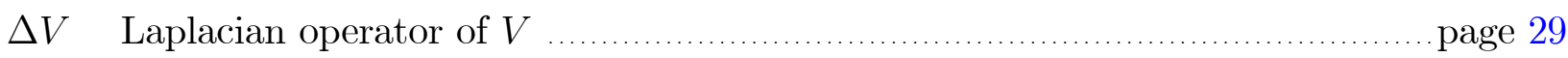

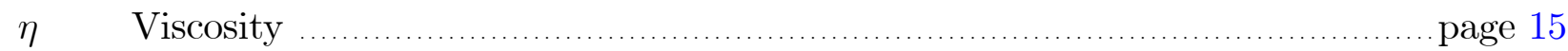

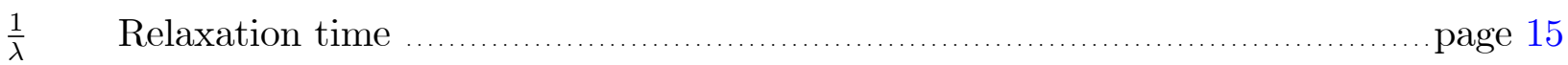

$\frac{\partial \phi}{\partial t} \quad$ Partial derivative of the function $\phi$ with respect to time $t \ldots \ldots \ldots \ldots \ldots \ldots \ldots \ldots \ldots$ page 8

$\frac{D \phi}{D t} \quad$ Material derivative of the mapping $\phi$ defined as $\frac{D \phi}{D t} \equiv \frac{\partial \phi}{\partial t}+V \cdot \nabla \phi \ldots \ldots \ldots \ldots \ldots$ page 8

$\Gamma \quad$ Boundary of the domain $\Omega$ (alternative notation for $\partial \Omega$ ) ....................... page 22

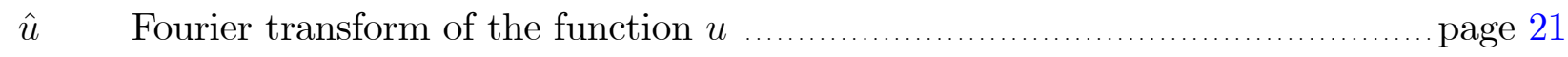

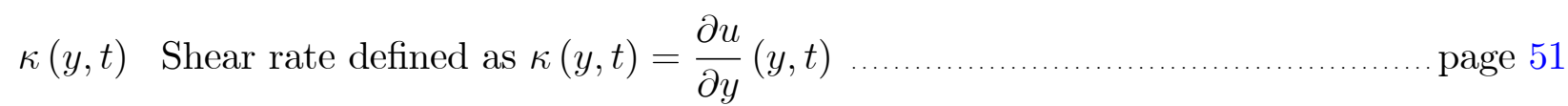

$\kappa^{n} \quad$ Approximate value of $\kappa$ at iteration $n$ of the scheme …........................ page 56

$\Lambda_{0 i}, i=1,2,3 \quad$ Eigenvalues of the initial extra stress tensor $\mathbf{T}_{0} \ldots \ldots \ldots \ldots \ldots \ldots \ldots \ldots \ldots \ldots$

$\lambda_{0} \quad$ Used in White-Metzner models, defined as $\lambda_{0}:=\lambda(0) \ldots \ldots \ldots \ldots \ldots \ldots \ldots \ldots \ldots \ldots$ page 80

$\lambda_{V} \quad$ Used in White-Metzner models, defined as $\lambda_{V}:=\lambda\left(\Pi_{D_{V}}\right)-\lambda(0) \ldots \ldots \ldots \ldots \ldots$ page 80

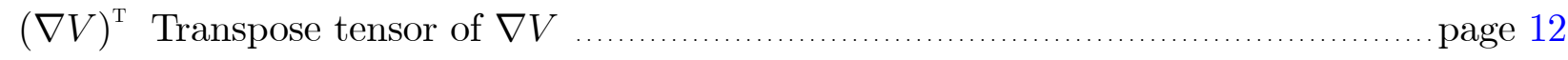


$[0, \mathrm{~T}] \quad$ Time interval, $\mathrm{T}>0$ page 16

$\|u\|_{X}$ Norm of the function $u$ in the space $X$ page 19

$\mathbb{N} \quad$ Set of positive integers or nonzero natural numbers page 29

$\mathbb{R} \quad$ Set of real numbers page 7

$\mathbb{R}^{m} \quad$ Euclidean space of $m$-dimentional page 6

$\tilde{\mathbf{f}} \quad$ Function defined as $\tilde{\mathbf{f}}=\mathbf{f}-\mathbf{f}_{0}$ page 70

$\tilde{\mathbf{T}} \quad$ Tensor defined as $\tilde{\mathbf{T}}=\mathbf{T}-\mathbf{T}_{0}$ page 70

$\tilde{\mathbf{T}}^{n} \quad$ Tensor defined as $\tilde{\mathbf{T}}^{n}=\mathbf{T}^{n}-\mathbf{T}_{0}$ in large data case, page 72 and defined as $\tilde{\mathbf{T}}^{n}=\mathbf{T}^{n}-\mathbf{T}^{n-1}$ in small data case page 46

f Body force acting on the fluid page 9

$\mathbf{T} \quad$ Extra stress tensor, $\mathbf{T} \equiv\left(T_{i j}\right)_{1 \leq i, j \leq 3}$ page 9

$\mathbf{T}^{n} \quad$ Approximate value of $\mathbf{T}$ at iteration $n$ of the scheme page 42

$\mathbf{T}_{0} \quad$ Initial extra stress tensor page 12

$\mathbf{x} \quad$ Space variable in $\mathbb{R}^{3}, \mathbf{x} \equiv\left(x_{1}, x_{2}, x_{3}\right)$ or $\mathbf{x} \equiv(x, y, z)$ page 7

$\mathcal{D}(\Omega) \quad$ Alternative notation for the space $C_{0}^{\infty}(\Omega)$ page 19

$\mathcal{D}^{\prime}(\Omega)$ Set of distributions or generalized function on $\Omega$ page 20

$\mathcal{V} \quad$ Space of all divergence free vector fields in $(\mathcal{D}(\Omega))^{3}$ page 21

$\mu \quad$ Elastic modulus page 12

$\mu_{0} \quad$ Used in White-Metzner models, defined as $\mu_{0}:=\mu(0) \ldots \ldots \ldots \ldots \ldots \ldots \ldots \ldots \ldots \ldots \ldots$ page 80

$\mu_{V} \quad$ Used in White-Metzner models, defined as $\mu_{V}:=\mu\left(I_{D_{V}}\right)-\mu(0)$ page 80

$\nabla p \quad$ Gradient of the scalar $p$ page 9 
$\Omega \quad$ Bounded open set in $\mathbb{R}^{m}, m=2,3$ page 6

curl Vector operator curl or rotor page 16

$\operatorname{div} V$ Divergence of the vector field $V$ page 8

$\bar{\Omega} \quad$ Closure of the domain $\Omega$ page 19

$\partial \Omega \quad$ Boundary of the domain $\Omega$ page 10

$\rho(\mathbf{x}, t) \quad$ Fluid mass density page 8

$\sigma \quad$ Total stress tensor page 9

$\tilde{p} \quad$ Scalar function defined as $\tilde{p}=p-p_{0}$ page 70

$\tilde{p}^{n} \quad$ Scalar function defined as $\tilde{p}^{n}=p^{n}-p_{0}$ in large data case, page 73 and defined as $\tilde{p}^{n}=p^{n}-p^{n-1}$ in small data case page 46

$\tilde{q} \quad$ Scalar function defined as $\tilde{q}=q-q^{0}$ page 71

$\tilde{q}^{n} \quad$ Scalar function defined as $\tilde{q}^{n}=q^{n}-q^{0}$ in large data case, page 72 and defined as $\tilde{q}^{n}=q^{n}-q^{n-1}$ in small data case page 46

$\tilde{V} \quad$ Vector field defined as $\tilde{V}=V-V^{0}$ page 71

$\tilde{V}^{n} \quad$ Vector field defined as $\tilde{V}^{n}=V^{n}-V^{0}$ in large data case, page 72 and defined as $\tilde{V}^{n}=V^{n}-V^{n-1}$ in small data case page 46

$\widetilde{\tilde{\mathbf{T}}}^{n} \quad$ Tensor defined as $\widetilde{\tilde{\mathbf{T}}}^{n}=\tilde{\mathbf{T}}^{n}-\tilde{\mathbf{T}}^{n-1}$ page 75

$\widetilde{\mathcal{V}} \quad$ Space of all divergence free vector fields in $H_{0}^{1}(\Omega)$ page 21

$\widetilde{\tilde{p}}^{n} \quad$ Scalar function defined as $\widetilde{\tilde{p}}^{n}=\tilde{p}^{n}-\tilde{p}^{n-1}$ page 75

$\widetilde{\tilde{q}}^{n} \quad$ Scalar function defined as $\widetilde{\tilde{q}}^{n}=\tilde{q}^{n}-\tilde{q}^{n-1}$ page 75 
$\widetilde{\tilde{V}}^{n} \quad$ Vector field defined as $\widetilde{\tilde{V}}^{n}=\tilde{V}^{n}-\tilde{V}^{n-1}$ page 75

$a \quad$ Acceleration in Eulerian description defined as $a=\frac{D V}{D t} \equiv \frac{\partial V}{\partial t}+V \cdot \nabla V$ page 8 $C\left([0, \mathrm{~T}] ; W^{s, r}(\Omega)\right) \quad$ Space of continuous $W^{s, r}(\Omega)$-valued functions on $[0, \mathrm{~T}]$ page 21

$C(\Omega) \quad$ Space of all continuous functions defined on $\Omega$ page 19

$C^{k}(\Omega)$ Set of functions $u \in C(\Omega)$ with $D^{\alpha} u \in C(\Omega)$ for $1<|\alpha| \leq k$ page 19

$C_{0}^{\infty}(\Omega)$ Space of all infinitely-differentiable functions of compact support in $\Omega$, elements of $C_{0}^{\infty}(\Omega)$ are called test functions page 19

$C_{b}^{k}(\Omega)$ Set of functions $u \in C^{k}(\Omega)$ with $D^{\alpha} u$ is bounded on $\Omega$ for $|\alpha| \leq k$ page 19

$D^{\alpha} \quad$ Partial derivatives defined as $D^{\alpha}=\frac{\partial^{|\alpha|}}{\partial x_{1} \partial x_{2} \ldots \partial x_{m}}$ page 19

$D_{V} \quad$ Strain tensor or symmetric gradient of the vector field $V$, defined as $D_{V}=\frac{1}{2}\left(\nabla V+(\nabla V)^{\mathrm{T}}\right)$ page 13

$g(\nabla V, \mathbf{T})$ Tensor valued mapping of second-order tensors page 12

$h \quad$ Distance between two parallel plates in simple shear flow page 14

$H^{s}(\Omega)$ Sobolev space of functions with weak derivatives up to order $s$ in $L^{2}(\Omega) \ldots$ page 21 $\Pi_{D_{V}} \quad$ Shear rate defined as $\Pi_{D_{V}}=\frac{1}{2} \operatorname{tr}\left(\left(D_{V}\right)^{2}\right) \equiv \frac{1}{2}\left|D_{V}\right|^{2}$ page 13

$L^{r}(\Omega)$ Class of all Lebesgue measurable functions $u$ defined on $\Omega$ with $\int_{\Omega}|u(\mathbf{x})|^{r} d \mathbf{x}<\infty$ page 20

$L_{0}^{2}(\Omega)$ Space of all functions $u \in L^{2}(\Omega)$ with $\int_{\Omega} u(\mathbf{x}) d \mathbf{x}=0$ page 21

$M: N$ Double dot product of matrices defined as $M: N=\sum_{i, j=1}^{m} M_{i j} N_{j i}$ page 24

$n \quad$ Unit outward normal vector, used also for positive integers $n \in \mathbb{N}$ page 15,19

$p \quad$ Pressure of the fluid page 9

$p^{n} \quad$ Approximate value of $p$ at iteration $n$ of the scheme page 42 
$q \quad$ Scalar function defined as $q=\left(\frac{\partial}{\partial t}+V \cdot \nabla+\lambda\right) p$ page 41

$q^{n} \quad$ Approximate value of $q$ at iteration $n$ of the scheme page 42

$s \quad$ Integer number $\geq 1$ or $\geq 0$ page 20,52

$t \quad$ Time variable $t \geq 0$ page 6

$u(y, t)$ First component of the velocity field vector in simple shear flow page 14

$V \quad$ Velocity field $V \in \mathbb{R}^{m}, m=2,3$ page 7

$V^{n} \quad$ Approximate value of $V$ at iteration $n$ of the scheme page 42

$W^{s, r}(\Omega) \quad$ Sobolev space of functions with weak derivatives up to order $s$ in $L^{r}(\Omega)$.. page 20 $W_{0}^{k, r}(\Omega)$ Sobolev space of all functions of $W^{s, r}(\Omega)$ with zero boundary values page 21 $\operatorname{tr}\left(\left(D_{V}\right)^{2}\right)$ Trace of the tensor $\left(D_{V}\right)^{2}$ page 13 


\section{Introduction}

$\mathrm{V}$

iscoelastic fluids, such as egg whites, liquid soap, liquid polymers, oils etc., are classified as non-Newtonian fluids with memory. That is, in addition to the current velocity field, the extra stress also depends on the history of previous deformations [29], [21], [25]. For linear viscoelasticity, Maxwell proposed a linear constitutive model by connecting in series linear viscous and elastic elements [21], [16]. The mathematical representation for this model is a linear differential equation relating the extra stress tensor with the velocity gradient. Many widely used models are based on adding nonlinear terms to the Maxwell model. We shall refer to these models as Maxwell-type differential constitutive equations [21], which are written in the following form:

$$
\frac{\partial \mathbf{T}}{\partial t}+(V \cdot \nabla) \mathbf{T}+\lambda \mathbf{T}=\mu\left(\nabla V+(\nabla V)^{\mathrm{T}}\right)+g(\nabla V, \mathbf{T}),
$$

where $g(\nabla V, \mathbf{T})$ is a tensor valued mapping of second-order tensors.

These constitutive equations are laws describing the properties and behavior of the fluid, they are crucial for solving uniquely the balance of momentum equation

$$
\rho\left(\frac{\partial V}{\partial t}+V \cdot \nabla V\right)=-\nabla p+\operatorname{div} \mathbf{T}-\mathbf{f} \text { in } \Omega \times[0, \mathrm{~T}], \mathrm{T}>0,
$$

with the incompressibility condition

$$
\operatorname{div} V=0 \text { in } \Omega \times[0, \mathrm{~T}],
$$

subject to the boundary condition

$$
V=0 \text { on } \partial \Omega \times[0, \mathrm{~T}]
$$


and the initial conditions

$$
\begin{gathered}
V(\cdot, 0)=0 \text { in } \Omega, \\
\mathbf{T}(\cdot, 0)=\mathbf{T}_{0} \text { in } \Omega .
\end{gathered}
$$

The problem of the equations (0.1)-(0.6) can be found in the literature with the name unsteady flow problem of viscoelastic Maxwell fluids, which has the unknown variables of extra stress tensor $\mathbf{T}$, velocity field $V$, and pressure $p$. Many results of well-posedness are well known and have been established, see [28, 10, 11, 23].

The assumption of creeping flow is made for many classes of viscoelastic flow problems, especially in very viscous fluids where the inertial effects are very small compared to viscosity ones. Such flow problems, called quasistatic, are also known as very low Reynolds number flows [8]. The fluid inertia terms can be neglected and the left hand side of the equation (0.2) can be ignored. The balance of momentum equation is reduced, in this case, to the following equation:

$$
\operatorname{div} \mathbf{T}-\nabla p=\mathbf{f}
$$

We refer to the problem of the equations (0.7), (0.3) and (0.1) subject to the boundary condition (0.4) and the initial condition (0.6) as unsteady flow problem of a quasistatic viscoelastic Maxwell fluid. Our goal in this thesis is to examine the well-posedness of this problem. To prove the existence of the solution, we use an iterative method introduced by Renardy in [26], [27], [29] for steady flow problems. This method transforms our problem to iterative linear subproblems. At each iteration we solve an elliptic problem and two hyperbolic problems. The limit of the iteration provides the solution of our original problem. Here we present the results for the following cases:

- Three-dimensional flow, in a bounded domain, locally and globally in time for sufficiently small initial data.

- Simple shear flow, i.e. a spatially one-dimensional problem, locally in time with arbitrary initial data.

- Some three-dimensional flow problems, in a bounded domain, locally in time with large initial data. 
Also, we present results for models of White-Metzner type where the constants $\lambda$ and $\mu$ of the constitutive equation (0.1) are regarded as nonlinear functions depending on the term $\left|\frac{1}{2}\left(\nabla V+(\nabla V)^{\mathrm{T}}\right)\right|^{2}$, in three-dimensional case, locally and globally in time, with sufficiently small initial data.

"It might seem that the quasistatic case should be easier; in fact some of the very first existence results for initial value problems in viscoelasticity were for quasistatic deformation of viscoelastic solids [3]. However, for large initial data, the local well-posedness of the initial value problem depends on the ellipticity and unique solvability of a nonlinear system of PDEs. While conditions for ellipticity are relatively easy to analyze, the unique solvability poses a challenge which, to our knowledge, remains unsolved for any model of viscoelastic flow." Michael Renardy

In our analysis below, we shall assume spatially uniform initial stresses; in this case, unique solvability follows from ellipticity.

This thesis is organized as follows:

We start with a preliminary chapter to provide important definitions, and briefly present the physical problem motivating the mathematical work presented in this thesis.

The second chapter is devoted to presenting a brief review of some of the mathematical concepts that will be used in this thesis.

The third chapter is motivated by a technique used in this thesis, a technique that reduces a quasi-linear problem to a sequence of Stokes and evolution problems. The first section of chapter three is devoted to stating the result concerning Stokes problem. In the second section, we state a result with the assumptions needed to solve an evolution problem in hyperbolic case. Then we apply this result to a particular case and establish estimates we use in the subsequent chapters.

In chapter four, we prove the existence and uniqueness of solutions for a three-dimensional unsteady flow problem of a quasistatic viscoelastic Maxwell fluid, locally and globally in time, 
with sufficiently small initial data. The proof is based on the iterative method mentioned above.

In chapter five, we study the well-posedness for an unsteady simple shear flow problem, locally in time, in the case where the initial data are arbitrary.

In the sixth chapter, we present some results of existence and uniqueness for three-dimensional flow, locally in time, with arbitrary initial data.

In the seventh and final chapter, we present results for models of White-Metzner type in the three-dimensional case, locally and globally in time, with sufficiently small initial data.

We ultimately conclude our study, and offer additional perspectives for future research. 


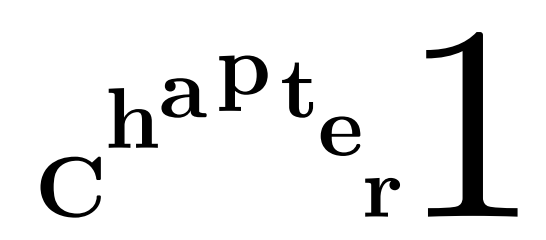

\section{Presentation of the problem}

\section{Contents}

1.1 Some basic concepts of fluid dynamics . . . . . . . . . 6

1.1.1 Representation of the motion . . . . . . . . . . 6

1.1.2 Three-, two- and one-dimensional flows $\ldots \ldots \ldots 6$

1.1 .3 Velocity field . . . . . . . . . . . . . . . 7

1.1.4 Steady and unsteady flow . . . . . . . . . . . 7

1.1.5 Material derivative and fluid acceleration . . . . . . . . . 8

1.2 Fundamental equations of fluid dynamics . . . . . . . 8

1.2.1 Conservation of mass . . . . . . . . . . . . . . . 8

1.2 .2 Balance of momentum . . . . . . . . . . . . . 9

1.2 .3 Constitutive equations . . . . . . . . . . . . . . . . . 10

1.2.4 Newtonian and non-Newtonian fluids . . . . . . . . . . . . . 10

1.2.5 Boundary and initial conditions . . . . . . . . . . . . . 10

1.3 Viscoelastic fluid flow . . . . . . . . . . . . . 11

1.3.1 Quasistatic or creeping viscoelastic flow . . . . . . . . . . . . 11 
1.3.2 Viscoelastic constitutive models . . . . . . . . . . . . . . . . 11

1.3.3 Viscoelastic simple shear flow . . . . . . . . . . . . . . . . . 14

1.4 Statement of the problem ............... 15

1.4.1 Physical description of the problem . . . . . . . . . . 15

1.4.2 Mathematical formulation . . . . . . . . . . . . . . 16

The purpose of this preliminary chapter is to provide important definitions, and briefly present the physical problem motivating the mathematical work presented in this thesis.

\subsection{Some basic concepts of fluid dynamics}

Details for this section can be found in many references on fluid mechanics, see for instance [8], [25], [15].

A fluid such as liquids and gases is a material body that can flow and easily change its shape, which fills a domain $\Omega_{0}$ in the space $\mathbb{R}^{3}$ at reference time $t=0$. At time $t>0$, the particles of the fluid occupy a region denoted by $\Omega$.

\subsubsection{Representation of the motion}

In fluid dynamics, the motion of a fluid can be described using two different methods. One is the Lagrangian method, which consists in following the movement of each particle. The other is the Eulerian method, which records the changes of velocity, acceleration, pressure and other characteristics at a fixed position.

\subsubsection{Three-, two- and one-dimensional flows}

For most problems of fluid mechanics, the flow is three-dimensional, where the mapping $\phi$ that characterizes a property of the fluid is a three-dimensional valued function, and each of 
its components is a function of space variable $\mathbf{x} \equiv\left(x_{1}, x_{2}, x_{3}\right) \in \mathbb{R}^{3(1)}$ and time variable $t$ in unsteady case.

In two-dimensional flow, such as when a fluid is placed between two parallel plates, the mapping $\phi$ describing a property of the fluid is a function of the form

$$
\phi \equiv\left(\phi_{1}\left(x_{1}, x_{2}, t\right), \phi_{2}\left(x_{1}, x_{2}, t\right)\right) \in \mathbb{R}^{2}, t \geq 0, x_{1}, x_{2} \in \mathbb{R}
$$

One-dimensional flow, such as a fluid moving in a tube, is the simplest case of flows. All corresponding fluid properties are expressed only as scalar functions of one space variable $x \in \mathbb{R}$ and time variable $t$.

\subsubsection{Velocity field}

In the Langrangian description, the position vector at time $t$ of a fluid particle whose initial position $\mathbf{x}_{0}$, is expressed as a function $M\left(\mathbf{x}_{0}, t\right)$. In this description, the velocity field $V\left(\mathbf{x}_{0}, t\right)$ is given by the time partial derivative of $M\left(\mathbf{x}_{0}, t\right)$.

In the Eulerian description, at each time $t$, the fluid flow is characterized by the velocity field observed at a fixed domain. This description is preferred in many fluid flow problems, and it is used in our study as well.

\subsubsection{Steady and unsteady flow}

Fluid flow can be classified as steady or unsteady. In the Eulerian description, the flow is called steady if the velocity field at every fixed point in space is independent of time. If it changes with time, the flow is said to be unsteady. The dependence of the velocity field on time variable makes the study of unsteady flows, in general, more difficult than the steady ones.

\footnotetext{
${ }^{(1)}$ An alternative notation is often used $\mathbf{x} \equiv(x, y, z)$.
} 


\subsubsection{Material derivative and fluid acceleration}

Suppose that $\phi=\phi(\mathbf{x}, t), \mathbf{x} \in \Omega \subset \mathbb{R}^{3}$ is a property (as density, pressure, ...) of a moving fluid with velocity $V$. The material derivative represents the time rate of change of the mapping $\phi$ and defined by

$$
\frac{D \phi}{D t} \equiv \frac{\partial \phi}{\partial t}+V \cdot \nabla \phi
$$

The acceleration of a fluid particle is the change in velocity field over the change in time. In the case for which the mapping $\phi$ is the velocity field $V$, the material derivative expression is the acceleration in the Eulerian description.

$$
a=\frac{D V}{D t} \equiv \frac{\partial V}{\partial t}+V \cdot \nabla V
$$

This expression is most commonly used in fluid mechanics. In the Lagrangian description, the acceleration is simply the partial derivative of the fluid velocity with respect to time variable.

\subsection{Fundamental equations of fluid dynamics}

In this section we present the basic equations describing the motion of a fluid. For more details on this subject, the reader is referred to books related to fluid mechanics, see for example [8], [25], [15].

\subsubsection{Conservation of mass}

The conservation of mass principle states that the total change of mass in a fixed material volume will remain unchanged over time. If $\rho(\mathbf{x}, t)$ denotes the fluid mass density, the law of conservation of mass, which is called the continuity equation, can be expressed as

$$
\frac{D \rho}{D t}+\rho \nabla \cdot V=0
$$

When the mass density remains unchanged during the fluid motion, the flow is called incompressible, and the continuity equation is reduced to the condition

$$
\nabla \cdot V=0 \text { or } \operatorname{div} V=0
$$


which is known as the incompressibility condition.

\subsubsection{Balance of momentum}

The balance of momentum equation for fluids can be derived from Newton's second law, which states that the time rate of change of the momentum is equal to the sum of forces acting on the fluid. It can be written as

$$
\rho \frac{D V}{D t}=\nabla \cdot \sigma-\mathbf{f}
$$

where $\sigma$ is the 'total' stress tensor and $\mathbf{-} \mathbf{f}$ is the body force acting on the fluid. The stress tensor $\sigma$ can be split into a pressure part $-p \mathbf{1}$ and an extra stress tensor $\mathbf{T}$ due to viscous forces, of the form

$$
\sigma=-p \mathbf{1}+\mathbf{T}
$$

where $p$ is the pressure of the fluid and $\mathbf{1}$ denotes the identity tensor. The momentum equation can be rewritten as

$$
\rho \frac{D V}{D t}=-\nabla p+\nabla \cdot \mathbf{T}-\mathbf{f} .
$$

An alternative form for this equation that is used is

$$
\rho\left(\frac{\partial V}{\partial t}+V \cdot \nabla V\right)=-\nabla p+\operatorname{div} \mathbf{T}-\mathbf{f} .
$$

The extra stress tensor $\mathbf{T}$ has the form

$$
\mathbf{T}=\left(\begin{array}{ccc}
T_{11} & T_{12} & T_{13} \\
T_{21} & T_{22} & T_{23} \\
T_{31} & T_{32} & T_{33}
\end{array}\right)
$$




\subsubsection{Constitutive equations}

For incompressible fluids, where the density $\rho$ and the body forces $\mathbf{-} \mathbf{f}$ are assumed to be given, the balance of momentum equation (1.2) with the conservation of mass equation (1.1) represent a system of four scalar equations for thirteen unknown scalar variables, of which only ten are independent due to symmetry of the tensor $\mathbf{T}$, which is required by the balance of angular momentum [31, Section 2.3]. It is thus necessary to complete this system with additional equations in order to have as many equations as unknowns, to be able to solve the problem. These supplementary equations, called constitutive equations or rheological equations of state [21, page 492], [22, Section 4.1], are laws describing the properties and behavior of the fluid. They relate the tensor $\mathbf{T}$ with the velocity $V$. Constitutive equations are crucial for solving the balance equations uniquely. Many constitutive models are proposed for different types of fluids. In the next section we present some of the most popular constitutive viscoelastic models.

\subsubsection{Newtonian and non-Newtonian fluids}

Depending on the constitutive relationship relating the extra stress tensor $\mathbf{T}$ with the velocity $V$, viscous fluids can be classified into two main types known as Newtonian and non-Newtonian fluids. In Newtonian fluids, the extra stress tensor is linearly dependent on the velocity gradient. However, when the velocity gradient and the extra stress tensor are related by a nonlinear mapping, the fluid is called non-Newtonian.

\subsubsection{Boundary and initial conditions}

In many fluid mechanics problems, particularly three-dimensional flows, we are interested in fluids flowing in a bounded domain $\Omega$. The assumptions that specify how the fluid behaves in the boundary $\partial \Omega$ are called boundary conditions. They are needed to determine some constants that appear when solving the balance and constitutive equations. The most common types of boundary conditions that can be imposed on $\partial \Omega$ are Neumann and Dirichlet conditions, or a combination of both. For a fluid bounded by a fixed solid, the velocity at 
the boundary is prescribed by homogeneous Dirichlet boundary condition

$$
V=0 \text { on } \partial \Omega \text {. }
$$

For unsteady flow problems, in addition to boundary conditions, we need to specify an additional condition, describing the state of the fluid throughout the domain $\Omega$ at initial time $t=0$, which is called initial condition.

\subsection{Viscoelastic fluid flow}

Fluids having both viscous and elastic properties, such as egg whites, liquid soap, liquid polymers, oils, glycerine etc., are called viscoelastic fluids. The main characteristic they have is the shape memory effect; that is, when a stress is applied on them, the original state is partially recovered after removing the stress.

For more details on the subject of this section, the reader can be referred to [29], [21], [25].

\subsubsection{Quasistatic or creeping viscoelastic flow}

In many classes of viscoelastic flow problems, the inertia effects are dominated by the ones of viscosity. Such flow problems, called quasistatic, are also known as very low Reynolds number or creeping flows [8]. The fluid inertia terms can be neglected and the left hand side of the equation (1.2) can be ignored. The balance of momentum equation is reduced, in this case, to the following equation:

$$
\operatorname{div} \mathbf{T}-\nabla p=\mathbf{f} .
$$

\subsubsection{Viscoelastic constitutive models}

As we mentioned in the Subsection 1.2.3, the balance equations have to be completed by constitutive equations, which describe the fluid properties.

Viscoelastic fluids are classified as non-Newtonian fluids with memory. That is, in addition to the current velocity field, the extra stress also depends on the history of previous deforma- 
tions. For linear viscoelasticity, Maxwell proposed a linear constitutive model by connecting in series linear viscous and elastic elements. The mathematical representation for this model is a linear ordinary differential equation relating the extra stress tensor with the velocity gradient

$$
\frac{\partial \mathbf{T}}{\partial t}+\lambda \mathbf{T}=\mu\left(\nabla V+(\nabla V)^{\mathrm{T}}\right)
$$

subject to an initial condition imposed the extra stress

$$
\mathbf{T}(\mathbf{x}, 0)=\mathbf{T}_{0}(\mathbf{x}), \mathbf{x} \in \Omega \subset \mathbb{R}^{3},
$$

where $\lambda \in \mathbb{R}, \mu>0$ and $(\nabla V)^{\mathrm{T}}$ represents the transpose tensor of $\nabla V$.

Based on the linear viscoelastic Maxwell model, many models are derived by adding nonlinear terms. The new models constructed are called generalized Maxwell models, whose constitutive equations are written in the following form known as Maxwell-type differential constitutive equations [21].

$$
\frac{\partial \mathbf{T}}{\partial t}+(V \cdot \nabla) \mathbf{T}+\lambda \mathbf{T}=\mu\left(\nabla V+(\nabla V)^{\mathrm{T}}\right)+g(\nabla V, \mathbf{T})
$$

where $g(\nabla V, \mathbf{T})$ is a tensor valued mapping of second-order tensors.

We present here, the function $g$ for a list of the most common of those generalized models:

- Upper Convected Maxwell model (UCM)

$$
g(\nabla V, \mathbf{T})=(\nabla V) \mathbf{T}+\mathbf{T}(\nabla V)^{\mathrm{T}}
$$

- Lower Convected Maxwell model (LCM)

$$
g(\nabla V, \mathbf{T})=-(\nabla V)^{\mathrm{T}} \mathbf{T}-\mathbf{T}(\nabla V) .
$$

- Corotational Maxwell model (CRM)

$$
g(\nabla V, \mathbf{T})=\frac{1}{2}\left(\nabla V-(\nabla V)^{\mathrm{T}}\right) \mathbf{T}-\frac{1}{2} \mathbf{T}\left(\nabla V-(\nabla V)^{\mathrm{T}}\right) .
$$


- Johnson-Segalman model (also called Interpolated Maxwell model)

$$
\begin{aligned}
g(\nabla V, \mathbf{T})= & (\nabla V) \mathbf{T}+\mathbf{T}(\nabla V)^{\mathrm{T}} \\
& -\frac{\nu}{2}\left[\left(\nabla V+(\nabla V)^{\mathrm{T}}\right) \mathbf{T}+\mathbf{T}\left(\nabla V+(\nabla V)^{\mathrm{T}}\right)\right] .
\end{aligned}
$$

Many other viscoelastic constitutive models that are nonlinear in the extra stress $\mathbf{T}$, expressed in the form of Maxwell-type (1.7) have been proposed [21], [29]:

- Phan-Thien Tanner model (PTT)

$$
g(\nabla V, \mathbf{T})=(\nabla V) \mathbf{T}+\mathbf{T}(\nabla V)^{\mathrm{T}}-\nu(t r \mathbf{T}) \mathbf{T}
$$

- Giesekus model

$$
g(\nabla V, \mathbf{T})=(\nabla V) \mathbf{T}+\mathbf{T}(\nabla V)^{\mathrm{T}}-\nu \mathbf{T}^{2}
$$

For all above viscoelastic constitutive models, as well as several other models proposed in the literature, the corresponding mapping $g$ is quadratic with no linear terms and has vanishing gradient at the origin.

\section{White-Metzner model}

Fluids of White-Metzner type are nonlinear models obtained by modifying the Maxwell-type constitutive equations, in such a way that the constants $\lambda$ and $\mu$ are regarded as nonlinear functions depending on the second invariant

$$
\Pi_{D_{V}}=\frac{1}{2} \operatorname{tr}\left(\left(D_{V}\right)^{2}\right) \equiv \frac{1}{2}\left|D_{V}\right|^{2}
$$

of the rate of strain tensor

$$
D_{V}=\frac{1}{2}\left(\nabla V+(\nabla V)^{\mathrm{T}}\right) .
$$

The quantity $\Pi_{D_{V}}$ is also called shear rate. Thus, White-Metzner models can be described by the constitutive equation [12], [33], [20]

$$
\frac{\partial \mathbf{T}}{\partial t}+(V \cdot \nabla) \mathbf{T}+\lambda\left(\Pi_{D_{V}}\right) \mathbf{T}=\mu\left(\Pi_{D}\right)\left(\nabla V+(\nabla V)^{\mathrm{T}}\right)+g(\nabla V, \mathbf{T}) .
$$


Example of White-Metzner models. For a class of viscoelastic fluids, the functions $\lambda\left(\Pi_{D_{V}}\right)$ and $\mu\left(\Pi_{D_{V}}\right)$ are given by [7], [14]

$$
\left.\begin{array}{l}
\lambda\left(\Pi_{D_{V}}\right)=\lambda_{0}\left(1+a \Pi_{D_{V}}\right)^{\beta}, \\
\mu\left(\Pi_{D_{V}}\right)=\mu_{0}\left[\left(1+a \Pi_{D_{V}}\right)^{\beta-\alpha}+b \Pi_{D_{V}}\left(1+a \Pi_{D_{V}}\right)^{-\beta-\alpha}\right],
\end{array}\right\}
$$

where $\lambda_{0}, \mu_{0}, a, b, \alpha$ and $\beta$ are constants characterizing the fluid.

\subsubsection{Viscoelastic simple shear flow}

Simple shear flow is a significant and simple type of flow. The fluid in this flow is placed between two parallel plates separated by a distance $h$, see Figure 1.1.

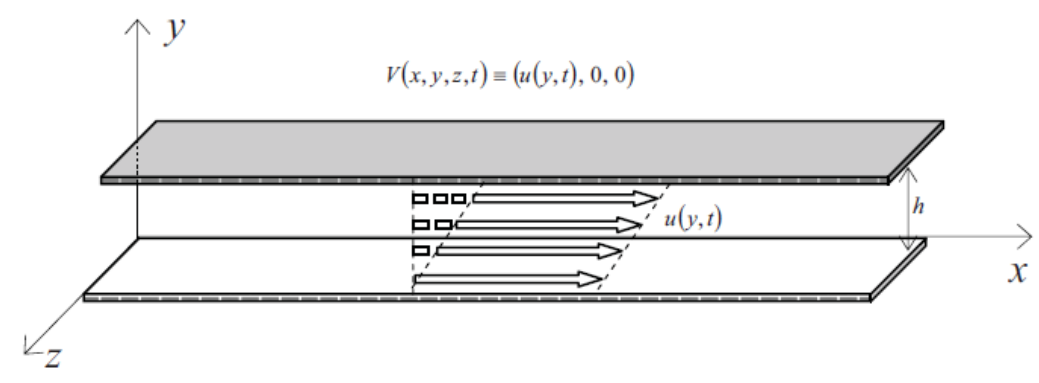

Figure 1.1: Simple shear flow: A fluid placed between two parallel plates.

The velocity of the fluid is only in the $x$-direction and changing only in the $y$-direction. Also, it depends on time $t$ for fluids in unsteady flow.

$$
V=(u(y, t), 0,0)
$$

The four components $T_{13}, T_{23}, T_{31}$ and $T_{32}$ of the extra stress tensor $\mathbf{T}$ are zero. Due to the symmetry of $\mathbf{T}$, only four non-zero independent components remain. Thus, $\mathbf{T}$ can be rewritten as

$$
\mathbf{T}=\left(\begin{array}{ccc}
T_{11}(y, t) & T_{12}(y, t) & 0 \\
T_{12}(y, t) & T_{22}(y, t) & 0 \\
0 & 0 & T_{33}(y, t)
\end{array}\right)
$$




\subsection{Statement of the problem}

In this section, we briefly describe the physical model and formulate the corresponding mathematical problem, which is a set of partial differential equations.

\subsubsection{Physical description of the problem}

Consider the case of a quasistatic viscoelastic ${ }^{(1)}$ incompressible $^{(2)}$ fluid flowing in a threedimensional bounded domain $\Omega$ with an impenetrable, fixed smooth boundary $\partial \Omega$ and unit outward normal vector $n$, see Figure 1.2.

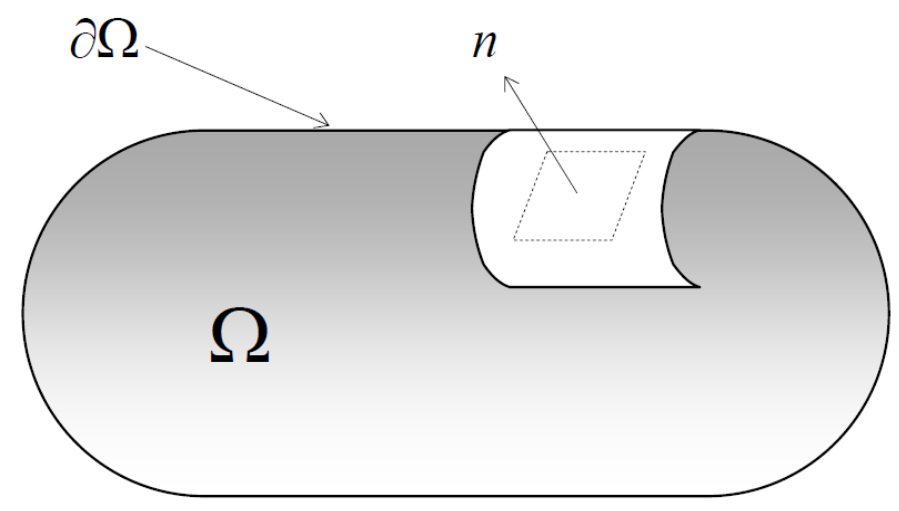

Figure 1.2: Fluid flow domain

The velocity field $V$ vanishes at the boundary $\partial \Omega$ since it is impenetrable and fixed. The extra stress measured at initial time $t=0$ will be denoted by $\mathbf{T}_{0}(\mathbf{x}), \mathbf{x} \in \Omega$. The viscoelastic fluid flowing in $\Omega$ is characterized by viscosity $\eta$, elastic modulus $\mu$, and the ratio of viscosity to elasticity $\frac{\eta}{\mu}$, which is called relaxation time and denoted by $\frac{1}{\lambda}$. We will restrict ourselves to unsteady $^{(3)}$ flow case with constitutive models of Maxwell-type ${ }^{(4)}$ and White-Metzner type ${ }^{(5)}$.

\footnotetext{
(1) See Section 1.3 page 11.

${ }^{(2)}$ See Subsection 1.2.1 page 8 .

${ }^{(3)}$ See Subsection 1.1.4 page 7 .

${ }^{(4)}$ See Subsection 1.3.2, page 11.

${ }^{(5)}$ See Subsection 1.3.2, page 13.
} 
Our objective is to study the basic properties of the governing equations, which have the unknown variables of extra stress tensor $\mathbf{T}(\mathbf{x}, t)$, velocity field $V(\mathbf{x}, t)$, and pressure $p(\mathbf{x}, t)$ over $\Omega$ during the time interval $(0, \mathrm{~T}], \mathrm{T}>0$.

\subsubsection{Mathematical formulation}

Based on the physical description presented in the previous subsection and on the set of partial differential equations (1.4), (1.1), (1.3), (1.7) and (1.6) derived from the fundamental equations of fluid dynamics, the model problem describing an unsteady flow of a quasistatic viscoelastic fluid in three-dimensional geometry can be stated as follows: Find a velocity vector field $V \equiv\left(V_{1}, V_{2}, V_{3}\right)$, an extra stress tensor $\mathbf{T} \equiv\left(T_{i j}\right)_{1 \leq i, j \leq 3}$ and a scalar pressure $p$ satisfying the equations

$$
\begin{aligned}
& \operatorname{div} \mathbf{T}-\nabla p=\mathbf{f} \quad \text { in } \Omega \times[0, \mathrm{~T}] \\
& \operatorname{div} V=0 \quad \text { in } \Omega \times[0, \mathrm{~T}], \\
& V=0 \quad \text { on } \partial \Omega \times[0, \mathrm{~T}], \\
& \frac{\partial \mathbf{T}}{\partial t}+(V \cdot \nabla) \mathbf{T}+\lambda \mathbf{T}=\mu\left(\nabla V+(\nabla V)^{\mathrm{T}}\right)+g(\nabla V, \mathbf{T}) \text { in } \Omega \times[0, \mathrm{~T}], \\
& \mathbf{T}(\cdot, 0)=\mathbf{T}_{0} \quad \text { in } \Omega,
\end{aligned}
$$

where $g(\nabla V, \mathbf{T})$ is a tensor valued mapping of second-order tensors which is assumed to be smooth with vanishing gradient with respect to $(\nabla V, \mathbf{T})$ at $(0,0)$. Recall that the equation (1.13) is known as incompressibility condition and (1.15) as constitutive equation, with constants $\lambda \in \mathbb{R}$ and $\mu>0$. In White-Metzner models, $\lambda$ and $\mu$ are nonlinear functions of $\Pi_{D_{V}}{ }^{(1)}$.

Remark 1.1 If substituting the initial value condition (1.16) into the equation (1.12) then taking curl operator and using the fact that $\operatorname{curl}(\nabla p(\cdot, 0))=0$, this yields the constraint condition

$$
\operatorname{curl}\left(\operatorname{div}\left(\mathbf{T}_{0}\right)-\mathbf{f}(\cdot, 0)\right)=0 .
$$

\footnotetext{
${ }^{(1)}$ See Subsection 1.3.2, page 13.
} 
The objective of the work presented in this thesis is to study the existence and uniqueness of solutions for the system of the partial differential equations (1.12)-(1.16). 


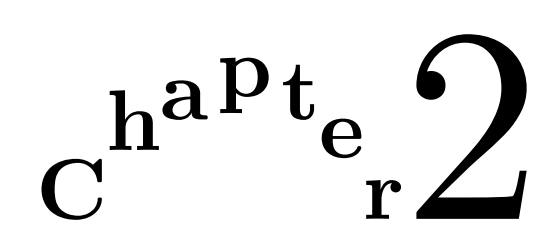

\section{Some basic mathematical concepts}

\section{Contents}

2.1 Notations and definitions . . . . . . . . . . . . . . 19

2.1 .1 Distributions . . . . . . . . . . . . . . . . . 19

2.1 .2 Sobolev spaces . . . . . . . . . . . . . . . . . 20

2.2 Basic results on Sobolev spaces . . . . . . . . . . . 22

2.2 .1 Approximation by smooth functions . . . . . . . . . . . 22

2.2.2 Trace and extension theorems . . . . . . . . . . . . . . . 22

2.2 .3 Divergence theorem $\ldots \ldots \ldots \ldots \ldots$

2.2.4 Sobolev embedding theorem . . . . . . . . . . . . . . . . . 24

2.2 .5 Chain rule........................... 25

2.3 Implicit function theorem $\ldots \ldots \ldots \ldots$

The main aim of this chapter is to present a brief review of some of the mathematical concepts that will be used in this thesis. For more details on this subject, the reader is referred to references cited throughout this chapter. 


\subsection{Notations and definitions}

We denote a vector in $\mathbb{R}^{m}$ by $\mathbf{x}=\left(x_{1}, x_{2}, \ldots, x_{m}\right)^{(1)}$. If $\alpha=\left(\alpha_{1}, \alpha_{2}, \ldots, \alpha_{m}\right)$ is $m$-dimensional multi-index, we set $|\alpha|=\alpha_{1}+\alpha_{2}+\ldots+\alpha_{m}, \alpha !=\alpha_{1} ! \alpha_{2} ! \ldots \alpha_{m} !$ and $\mathbf{x}^{\alpha}=x_{1}^{\alpha_{1}} x_{2}^{\alpha_{2}} \ldots x_{m}^{\alpha_{m}}$. The partial derivatives are denoted as follows:

$$
D^{\alpha}=\frac{\partial^{|\alpha|}}{\partial x_{1} \partial x_{2} \ldots \partial x_{m}} .
$$

We indicate by $\Omega$ a bounded open set in $\mathbb{R}^{m}(m=3)$ with smooth boundary $\partial \Omega$ and unit outward normal vector $n$. $C(\Omega)$ denotes the space of all continuous functions defined on $\Omega$. For any integer $k \geq 1$ let

$$
C^{k}(\Omega)=\left\{u \in C(\Omega) ; D^{\alpha} u \in C(\Omega) \text { for } 1<|\alpha| \leq k\right\}
$$

and

$$
C_{b}^{k}(\Omega)=\left\{u \in C^{k}(\Omega) ; D^{\alpha} u \text { is bounded on } \Omega \text { for }|\alpha| \leq k\right\} .
$$

The space $C_{b}^{k}(\Omega)$ is a Banach space for the norm

$$
\|u\|_{C_{b}^{k}(\Omega)}=\max _{0 \leq|\alpha| \leq k} \sup _{\mathbf{x} \in \Omega}\left|D^{\alpha} u(\mathbf{x})\right| .
$$

Let $\mathcal{D}(\Omega)^{(2)}$ be the space of all infinitely-differentiable functions of compact support in $\Omega$. The elements of $\mathcal{D}(\Omega)$ are called test functions. We set

$$
\mathcal{D}(\bar{\Omega})=\left\{\left.\phi\right|_{\Omega} ; \phi \in \mathcal{D}\left(\mathbb{R}^{m}\right)\right\}
$$

\subsubsection{Distributions}

We present in this subsection the definition of distributions and some basic operations that apply to them. For more details we refer the reader, for instance, to [30], [1].

Definition 2.1 (Distributions) A distribution or generalized function on $\Omega$ is a linear mapping $f$ from the space of test functions $\mathcal{D}(\Omega)$ to $\mathbb{R}$, which is continuous in the sense that if $\phi_{n} \rightarrow 0$ in $\mathcal{D}(\Omega)$, then $\left(f, \phi_{n}\right) \rightarrow 0$.

\footnotetext{
(1) If $m=3$, an alternative notation is often used $\mathbf{x}=(x, y, z)$.

${ }^{(2)}$ Alternative noation for $\mathcal{D}(\Omega)$ is $C_{0}^{\infty}(\Omega)$.
} 
The set of all distributions is denoted by $\mathcal{D}^{\prime}(\Omega)$. A sequence of distributions $\left(f_{n}\right)$ is said to converge in distributional sense to an element $f \in \mathcal{D}^{\prime}(\Omega)$, if $\left(f_{n}, \phi\right) \rightarrow(f, \phi)$ for every test function $\phi \in \mathcal{D}(\Omega)$.

Definition 2.2 (Derivatives) If $f \in \mathcal{D}^{\prime}(\Omega)$, the derivative of $f$ in the distributional sense with respect to $\mathbf{x}$ is defined by

$$
\left(D^{\alpha} f, \phi\right)=(-1)^{|\alpha|}\left(f, D^{\alpha} \phi\right), \phi \in \mathcal{D}(\Omega)
$$

Theorem 2.3 (Sequential Completeness of Distributions) Let $f_{n}$ be a sequence of distributions such that $\left(f_{n}, \phi\right)$ converges for every test function $\phi$. Then there exists $f$ in $\mathcal{D}^{\prime}(\Omega)$ such that $f_{n} \rightarrow f$.

\subsubsection{Sobolev spaces}

Let $r$ be a real real number $\geq 1$. We denote by $L^{r}(\Omega)$ the class of all Lebesgue measurable functions $u$ defined on $\Omega$ and satisfying the condition

$$
\int_{\Omega}|u(\mathbf{x})|^{r} d \mathbf{x}<\infty
$$

It is well known that the space $L^{r}(\Omega)$ endowed with the norm

$$
\|u\|_{L^{r}(\Omega)}=\left(\int_{\Omega}|u(\mathbf{x})|^{r} d \mathbf{x}\right)^{\frac{1}{r}},
$$

is a Banach space, and for $r=2$ is a Hilbert space.

Let now $s$ be an integer $\geq 1$. We define the Sobolev space

$$
W^{s, r}(\Omega)=\left\{u \in L^{r}(\Omega) ; D^{\alpha} u \in L^{r}(\Omega) \text { for }|\alpha| \leq s\right\},
$$

where $D^{\alpha} u$ is the partial distributional derivative of $u$. The space $W^{s, r}(\Omega)$ is a Banach space for the norm

$$
\|u\|_{W^{s, r}(\Omega)}=\left\{\sum_{|\alpha| \leq s}\left\|D^{\alpha} u\right\|_{L^{r}(\Omega)}\right\}^{\frac{1}{r}} .
$$


The closure of $\mathcal{D}(\Omega)$ in $W^{s, r}(\Omega)$ is denoted by $W_{0}^{s, r}(\Omega)$. When $r=2$, the notation $H^{s}(\Omega)$ is usually used for the space $W^{s, 2}(\Omega)$, which is a separable Hilbert space for the inner product

$$
(u, v)=\sum_{|\alpha| \leq s} \int_{\Omega} D^{\alpha} u(\mathbf{x}) D^{\alpha} v(\mathbf{x}) d \mathbf{x}
$$

We define some other useful spaces

$$
\begin{gathered}
\mathcal{V}=\left\{\phi \in(\mathcal{D}(\Omega))^{3}, \operatorname{div} \phi=0\right\}, \\
\widetilde{\mathcal{V}} \equiv \text { the closure of } \mathcal{V} \text { in } H_{0}^{1}(\Omega), \\
L_{0}^{2}(\Omega)=\left\{u \in L^{2}(\Omega), \int_{\Omega} u(\mathbf{x}) d \mathbf{x}=0\right\} .
\end{gathered}
$$

For real number $s>0$, we set

$$
H^{s}\left(\mathbb{R}^{m}\right)=\left\{u \in L^{2}\left(\mathbb{R}^{m}\right) ;\left(1+|\xi|^{2}\right)^{\frac{s}{2}} \hat{u}(\xi) \in L^{2}\left(\mathbb{R}_{\xi}^{m}\right)\right\}
$$

which is equipped with the norm

$$
\|u\|_{H^{s}\left(\mathbb{R}^{m}\right)}=\left\|\left(1+|\xi|^{2}\right)^{\frac{s}{2}} \hat{u}\right\|_{L^{2}\left(\mathbb{R}_{\xi}^{m}\right)},
$$

and make it a Hilbert Space, where $\hat{u}$ denotes the Fourier transform of $u$. For the domain $\Omega$ and $s$ is real number $>0$, we define

$$
H^{s}(\Omega)=\left\{u \in L^{2}(\Omega) ; \text { there is } \tilde{u} \in H^{s}\left(\mathbb{R}^{m}\right) \text { such that }\left.\tilde{u}\right|_{\Omega}=u\right\} \text {, }
$$

endowed with the norm

$$
\|u\|_{H^{s}(\Omega)}=\inf _{\tilde{u} \in H^{s}\left(\mathbb{R}^{m}\right),\left.\tilde{u}\right|_{\Omega}=u}\|\tilde{u}\|_{H^{s}\left(\mathbb{R}^{m}\right)} .
$$

Note that if $s$ is an integer and the boundary $\partial \Omega$ is smooth enough, then the definition (2.16) coincides with the definition in $(2.8)$ for $H^{s}(\Omega)$, see next section.

We use the same notation for scalar and tensor valued Sobolev Spaces $W^{s, r}(\Omega) \equiv\left\{W^{s, r}(\Omega)\right\}^{l}$, $l=1,2, \ldots$.

Let now $\mathrm{T}$ be a nonnegative real number. We denote the space of continuous Sobolev-spacesvalued functions defined on $[0, \mathrm{~T}]$ by $C\left([0, \mathrm{~T}] ; W^{s, r}(\Omega)\right)$, and if $r=2$ by $C\left([0, \mathrm{~T}] ; H^{s}(\Omega)\right)$. We 
use the same notation for tensor valued spaces $H^{s}(\Omega) \equiv\left\{H^{s}(\Omega)\right\}^{m}$ and $C_{b}\left([0, \mathrm{~T}] ; H^{s}(\Omega)\right) \equiv$ $C_{b}\left([0, \mathrm{~T}] ;\left\{H^{s}(\Omega)\right\}^{m}\right), m \in\{3,9\}$. When there is no ambiguity about the domain $\Omega$, we denote $\|\cdot\|_{H^{s}}$ and $\|\cdot\|_{C\left([0, \mathrm{~T}] ; H^{s}\right)}$ the norms in the spaces $H^{s}(\Omega)$ and $C\left([0, \mathrm{~T}] ; H^{s}(\Omega)\right)$, respectively.

\subsection{Basic results on Sobolev spaces}

In this section we present some of the main results concerning Sobolev spaces.

\subsubsection{Approximation by smooth functions}

The so called "density argument" is usually used to extend results established for smooth functions to some large spaces. Now we present some density results which can be found in [1], [9] and [30]:

- The space $\mathcal{D}(\Omega)$ is dense in $\mathcal{D}^{\prime}(\Omega)$.

- The space $\mathcal{D}(\Omega)$ is dense in $L^{r}(\Omega)$ for $1 \leq r<\infty$.

- The space $C^{\infty}(\Omega) \cap W^{s, r}(\Omega)$ is dense in $W^{s, r}(\Omega)$ for any integer $s \geq 1$ and $1 \leq r<\infty$.

- The space $\mathcal{D}(\bar{\Omega})$ is dense in $W^{s, r}(\Omega)$ for any integer $s \geq 1$ and $1 \leq r<\infty$.

- The space $\mathcal{D}\left(\mathbb{R}^{m}\right)$ is dense in $W^{s, r}\left(\mathbb{R}^{m}\right)$ for any integer $s \geq 1$ and $1 \leq r<\infty$.

\subsubsection{Trace and extension theorems}

Let $\Gamma=\partial \Omega$ be the boundary of $\Omega$ which is supposed to be smooth enough so that $\Gamma$ can be identified with a $m-1$ dimensional submanifold of $\mathbb{R}^{m}$. Let $\left(\mathcal{O}_{j}\right)_{1 \leq j \leq J}$ be a set of open bounded domains in $\mathbb{R}^{m}$ covering $\Gamma$ and let $\Phi_{j}: \mathcal{O}^{\prime} \subset \mathbb{R}^{m-1} \rightarrow \Gamma \cap \mathcal{O}_{j}$ the mapping associated. Then we set the following definition (see [9]). 
Definition 2.4 (of $W^{s, r}(\Gamma)$ ) The space $W^{s, r}(\Gamma)$ is the set of all distributions $u$ on $\Gamma$ such that $u \circ \Phi \in W^{s, r}\left(\mathcal{O}^{\prime} \cap \Phi^{-1}\left(\Gamma \cap \mathcal{O}_{j}\right)\right)$ for all possible $\Phi$ and $\mathcal{O}_{j}$.

Now, let $u$ be a function in the space $\mathcal{D}(\bar{\Omega})$ and let the mapping $\gamma: \mathcal{D}(\bar{\Omega}) \rightarrow\{\mathcal{D}(\Gamma)\}^{s}$ be defined by

$$
\gamma u \equiv\left(\gamma_{0} u, \gamma_{1} u, \ldots, \gamma_{s-1} u\right)=\left(\left.u\right|_{\Gamma},\left.\frac{\partial u}{\partial n}\right|_{\Gamma}, \ldots,\left.\frac{\partial^{s-1} u}{\partial n^{s-1}}\right|_{\Gamma}\right),
$$

where $\left.\frac{\partial^{j} u}{\partial n^{j}}\right|_{\Gamma}$ is the normal derivative of order $j$ on $\Gamma$, oriented toward the interior of $\Omega$.

Theorem 2.5 (Trace theorem [30], [9]) Let $\Omega$ be of class $C^{s}$ and let $\Gamma$ be bounded. The mapping $\gamma$ defined by (2.18) has a unique linear continuous extension, still denoted $\gamma$, of $H^{s}(\Omega) \rightarrow \prod_{j=0}^{s} H^{s-j-\frac{1}{2}}(\Gamma)$. Moreover, the mapping $\gamma$ is surjective, has a bounded right inverse and $\operatorname{ker}(\gamma)=H_{0}^{s}(\Omega)$.

Some results concerning Sobolev spaces are easy to prove in $W^{s, r}\left(\mathbb{R}^{m}\right)$. Many of these results still valid for the domain $\Omega$ if the functions of $W^{s, r}(\Omega)$ can be extended to $W^{s, r}\left(\mathbb{R}^{m}\right)$. Obviously functions of $L^{r}(\Omega)$ can be extended by zero to $L^{r}\left(\mathbb{R}^{m}\right)$. However, the following theorem is concerned with the extension of the functions of Sobolev spaces $W^{s, r}(\Omega)$ to $W^{s, r}\left(\mathbb{R}^{m}\right)$.

Theorem 2.6 (Extension theorem [1]; see also [30, Theorem 7.60 page 225]) Suppose that $\Omega \subset \mathbb{R}^{m}$ is a bounded domain with a boundary $\Gamma$ of class $C^{s}$. Then, for any real $r \geq 1$, there exists a linear bounded extension operator from $W^{s, r}(\Omega)$ into $W^{s, r}\left(\mathbb{R}^{m}\right)$. Conversely, the space $W^{s, r}(\Omega)$ coincides with the class of restrictions to $\Omega$ of the functions of $W^{s, r}\left(\mathbb{R}^{m}\right)$.

\subsubsection{Divergence theorem}

Let $V$ be a continuously differentiable vector field defined on a neighborhood of $\Omega$. In physics, the divergence theorem is a result which states that the total outward flux of the vector field $V$ through the closed boundary $\Gamma$ is the same as the integral of the divergence of the vector field $V$ on $\Omega$. The mathematical statement of this theorem is given by the following formula:

$$
\int_{\Omega} \operatorname{div} V d \mathbf{x}=\int_{\partial \Omega} V \cdot n d S
$$


which is a form of the fundamental theorem of calculus in three-dimensions, where $n$ is the unit outward normal vector of the surface $\partial \Omega$ and $d S$ is the surface area element.

Now, let $u$ be a continuously differentiable scalar function defined on a neighborhood of $\Omega$. By using the identity

$$
\operatorname{div}(u V)=V \cdot \nabla u+u \operatorname{div} V
$$

and applying the divergence theorem (2.19), we obtain the following identity:

$$
\int_{\Omega} u \operatorname{div} V d \mathbf{x}=\int_{\partial \Omega} u V \cdot n d S-\int_{\Omega} V \cdot \nabla u d \mathbf{x}
$$

which can be extended to the case where $u, V \in H^{s}(\Omega), s \geq 1$ using density arguments. It also can be generalized to the case where $M$ is a square matrix and $U$ is a vector field.

$$
\int_{\Omega}(\operatorname{div} M) \cdot U d \mathbf{x}=\int_{\partial \Omega} U^{\mathrm{T}} M \cdot n d S-\int_{\Omega} M:(\nabla U)^{\mathrm{T}} d \mathbf{x},
$$

where ":" denotes the double dot product of matrices $M: N=\sum_{i, j=1}^{m} M_{i j} N_{j i}$.

\subsubsection{Sobolev embedding theorem}

In this subsection, we state the theorem that is concerned with embedding Sobolev spaces into other spaces of functions.

Theorem 2.7 (Sobolev embedding theorem [1]) Assume that $\Omega$ has the cone property, see [1, page 66]. Let $r, r^{\prime}$ be real numbers $\geq 1$. Let $s$ be an integer $\geq 1$ and $j$ be positive integer. Then we have the following embeddings:

$$
\begin{gathered}
W^{j+s, r}(\Omega) \hookrightarrow W^{j, r^{\prime}}(\Omega) \quad \text { if } s r<m \text { and } r \leq r^{\prime} \leq \frac{m r}{m-s r}, \\
W^{s, r}(\Omega) \hookrightarrow L^{r^{\prime}}(\Omega) \quad \text { if } s r=m \text { and } r \leq r^{\prime}<\infty, \\
W^{j+s, 1}(\Omega) \hookrightarrow C_{b}^{j}(\Omega) \quad \text { if } s=m, \\
W^{j+s, r}(\Omega) \hookrightarrow C_{b}^{j}(\Omega) \quad \text { if } s>\frac{m}{r} .
\end{gathered}
$$

The following useful result follows from the Sobolev embedding theorem, see [35, Corollary 21.78 page 295$]$ and [1, Theorem 5.23 page 115$]$. 
Proposition 2.8 Let $r$ be real number $\geq 1$, let $s$ be an integer with $s r>m$, and let $l$ be positive integer. Then for all $u_{1}, u_{2}, \ldots, u_{l} \in W^{s, r}(\Omega)$, we have

$$
D^{\alpha_{1}} u_{1} D^{\alpha_{2}} u_{2} \ldots D^{\alpha_{l}} u_{l} \in L^{r}(\Omega) \text { for } \sum_{i=1}^{l}\left|\alpha_{i}\right| \leq s
$$

and

$$
u_{1} u_{2} \ldots u_{l} \in W^{s, r}(\Omega)
$$

\subsubsection{Chain rule}

The chain rule formula for the composition of multivariable smooth functions is known as multivariate Faà di Bruno's formula [5], which can be stated as follows.

Let $u(\mathbf{y})$ be a scalar-valued function of class $C^{s}$ on a domain $\mathcal{O} \subset \mathbb{R}^{d}$. Let $v(\mathbf{x})$ be a $\mathcal{O}$ valued function of class $C^{s}$ defined on the domain $\Omega \subset \mathbb{R}^{m}$. Then, for any $m$-dimensional multi-index $\alpha$ with $|\alpha| \leq s$, the partial derivative $D_{\mathbf{x}}^{\alpha}(u \circ v)(\mathbf{x})$ is given by

$$
D_{\mathbf{x}}^{\alpha}(u \circ v)(\mathbf{x})=\sum_{1 \leq|\beta| \leq|\alpha|} D_{\mathbf{y}}^{\beta} u(v(\mathbf{x})) \sum_{r=1}^{|\alpha|} \sum_{\sigma_{r}(\beta)}(\alpha !) \prod_{l=1}^{r} \frac{\left[D_{\mathbf{x}}^{\nu_{l}} v(\mathbf{x})\right]^{\kappa_{l}}}{\left(\kappa_{l} !\right)\left[\nu_{l} !\right]^{\left|\kappa_{l}\right|}},
$$

where, $\nu_{l}$ is $m$-dimensional multi-index, $\beta, \kappa_{l}$ are $d$-dimensional multi-indices and

$$
\sigma_{r}(\beta)=\left\{\left(\kappa_{1}, \ldots, \kappa_{r} ; \nu_{1}, \ldots, \nu_{r}\right) / \begin{array}{l}
\left|\kappa_{l}\right| \geq 1,0<\nu_{1}<\ldots<\nu_{r}, \\
\sum_{l=1}^{r} \kappa_{l}=\beta \text { and } \sum_{l=1}^{r}\left|\kappa_{l}\right| \nu_{l}=\alpha
\end{array}\right\}
$$

Note that we use here the convention $0^{0}=1$.

By the density of $C^{s}(\Omega) \cap W^{s, r}(\Omega)$ in $W^{s, r}(\Omega)$, the identity (2.24) can be extended to $v \in W^{s, r}(\Omega)$, provided that the product in the left hand side exists in the distributional sense.

As an application of the above chain rule formula, we have the following proposition.

Proposition 2.9 Assume that $\Omega \subset \mathbb{R}^{m}$ bounded domain with boundary $\Gamma=\partial \Omega$ smooth enough. Let $u: \mathbb{R}^{d} \rightarrow \mathbb{R}$ be a function of class $C^{s}, s>\frac{m}{2}$. Then, the function $u$ maps continuously $\left(H^{s}(\Omega)\right)^{d}$ into $H^{s}(\Omega)$. 
Proof. Let $v \in\left(H^{s}(\Omega)\right)^{d}$. Since $u \in C^{s}\left(\mathbb{R}^{d}\right)$ and $v \in\left(H^{s}(\Omega)\right)^{d} \hookrightarrow\left(C_{b}(\Omega)\right)^{d}$, then for any $d$-dimensional multi-index $\beta$ with $1 \leq|\beta| \leq s, D_{\mathbf{y}}^{\beta} u(v)$ is bounded on $\Omega$. On the other hand, by the formula (2.22), for all integer $r \geq 1$, we have $\prod_{l=1}^{r} \frac{\left[D_{\mathbf{x}}^{\nu_{l}} v(\mathbf{x})\right]^{\kappa_{l}}}{\left(\kappa_{l} !\right)\left[\nu_{l} !\right]^{\kappa_{l}} \mid} \in L^{2}(\Omega)$. Thus, the function $D_{\mathbf{x}}^{\alpha}(u \circ v) \in L^{2}(\Omega)$ for all $|\alpha| \leq s$. It follows that $u(v) \in H^{s}(\Omega)$. Using the same argument and by the continuity of the embedding $H^{s}(\Omega) \hookrightarrow C_{b}(\Omega)$, we prove the continuity of $u:\left(H^{s}(\Omega)\right)^{d} \rightarrow H^{s}(\Omega)$.

Corollary 2.10 If the function $u$ is of class $C^{s+2}, s>\frac{m}{2}$ with vanishing gradient at the origin, then $u$ and its derivatives up to order 2 map continuously $\left(H^{s}(\Omega)\right)^{d}$ into $H^{s}(\Omega)$ and the composite function $u(v(\mathbf{x})), v \in\left(H^{s}(\Omega)\right)^{d}$, satisfies a local Lipschitz condition of order 2 at the origin, in the sense that for given $c \geq 0$, we have

$$
\|u(v)-u(0)\|_{H^{s}} \leq L\|v\|_{\left(H^{s}\right)^{d}}^{2}
$$

for all $v \in\left(H^{s}(\Omega)\right)^{d}$ with $\|v\|_{\left(H^{s}\right)^{d}} \leq c$, where $L$ is a constant $>0$.

Proof. By the previous proposition, for all $|\beta| \leq 2, D_{\mathbf{y}}^{\beta} u$ map continuously $\left(H^{s}(\Omega)\right)^{d}$ into $H^{s}(\Omega)$. To establish the estimate (2.25), we use Taylor's formula with Lagrange's remainder in Banach Spaces.

$$
u(v)-u(0)=D_{\mathbf{y}}^{1} u(0)[v]^{1}+\frac{1}{2} D_{\mathbf{y}}^{2} u(h v)[v]^{2} .
$$

If $\|v\|_{\left(H^{s}\right)^{d}} \leq c, D_{\mathbf{y}}^{k} D_{\mathbf{y}}^{2} u(h v), k \leq s$ have a bound $L \geq 0$ depending only on $c$, then since $\nabla_{\mathbf{y}} u(0)=0$, we have

$$
\|u(v)-u(0)\|_{H^{s}} \leq L\left\|\sum_{1 \leq i, j \leq d} v_{i} v_{j}\right\|_{H^{s}} .
$$

Since $\left\|v_{i} v_{j}\right\|_{H^{s}} \leq\left\|v_{i}\right\|_{H^{s}}\left\|v_{j}\right\|_{H^{s}} \leq \frac{1}{2}\left(\left\|v_{i}\right\|_{H^{s}}^{2}+\left\|v_{j}\right\|_{H^{s}}^{2}\right)$ for every $v_{i}, v_{j}$ in $H^{s}(\Omega)$, we obtain the estimate required.

\subsection{Implicit function theorem}

In this section we state the inverse function theorem and its generalized form, which is called implicit function theorem. These theorems are useful in solving many nonlinear equations. 
Theorem 2.11 (Inverse function theorem). Let $E$ and $F$ be Banach spaces, let $\mathbf{x}_{0}$ be an interior point of an open set $U$ in $E$, and let $f: U \rightarrow F$ be $a C^{r}$ mapping, $r=1,2, \ldots$ Suppose that $D f\left(\mathbf{x}_{0}\right)$ is an invertible linear operator in $E$. Then there exists an open neighborhood $V$ of $\mathbf{x}_{0}$ in $E$ such that the restriction of $f$ to $V$ is one-to-one and the inverse function $\phi$ is $C^{r}$ diffeomorphism on the open set $W=f(V)$. If $\mathbf{x} \in V$ and $\mathbf{y}=f(\mathbf{x})$, then the following formula holds for the derivatives of $\phi$ at $\mathbf{y}$ :

$$
D \phi(\mathbf{y})=(D f(\mathbf{x}))^{-1}
$$

Theorem 2.12 (Implicit function theorem). Let $E, F$ and $G$ be Banach spaces, let $U_{0} \subseteq$ $E, V_{0} \subseteq F$ be open sets and let $f: U_{0} \times V_{0} \rightarrow G$ be a function of class $C^{r}, r=1,2, \ldots$ Suppose that $\left(\mathbf{x}_{0}, \mathbf{y}_{0}\right) \in U_{0} \times V_{0}$ with $f\left(\mathbf{x}_{0}, \mathbf{y}_{0}\right)=0$ and $D_{\mathbf{y}} f\left(\mathbf{x}_{0}, \mathbf{y}_{0}\right): F \rightarrow G$ has a bounded inverse operator, then there exist an open neighborhood $U \subseteq E$ of $\mathbf{x}_{0}$, an open neighborhood $V \subseteq F$ of $\mathbf{y}_{0}$, and a unique $C^{r}$ diffeomorphism $\phi: U \rightarrow V$ such that $f(\mathbf{x}, \phi(\mathbf{x}))=0$ for all $\mathbf{x} \in U$. Moreover, we have the following formula for the derivatives of $\phi:$

$$
D \phi(\mathbf{x})=-\left(D_{\mathbf{y}} f(\mathbf{x}, \phi(\mathbf{x}))\right)^{-1} \circ D_{\mathbf{x}} f(\mathbf{x}, \phi(\mathbf{x})) \text { for all } \mathbf{x} \in U \text {. }
$$

The above two theorems have different versions and their proof can be found in many books, see for example [30, Section 10.1.1].

Theorem 2.13 (Global inverse function theorem) Let $f: \mathbb{R}^{n} \rightarrow \mathbb{R}^{n}$ be a map of class $C^{r}$, $r=1,2, \ldots$. Then $f$ is a $C^{r}$ diffeomorphism if and only if

a) $D f(\mathbf{x})$ is an invertible linear operator in $\mathbb{R}^{n}$ for all $\mathbf{x} \in \mathbb{R}^{n}$.

b) $\lim _{\|\mathbf{x}\| \rightarrow \infty}\|f(\mathbf{x})\|=\infty$.

For the proof of this theorem see [34]. 


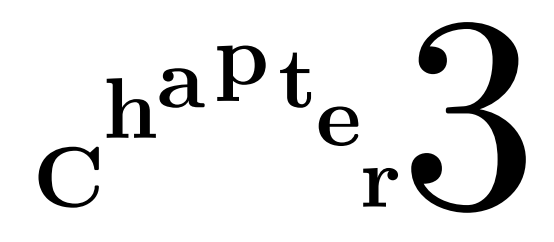

\section{Preliminary problems}

\section{Contents}

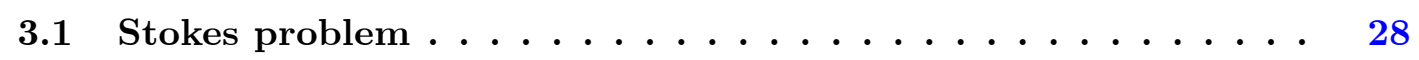

3.2 Evolution problem ........................ 29

This chapter is motivated by a technique used in this thesis, it is about a technique that reduces a quasi-linear problem to a sequence of Stokes and evolution problems. The first section of this chapter is devoted to stating the result concerning Stokes problem. In the second section, we state a result with the assumptions needed to solve an evolution problem in hyperbolic case. Then we apply this result to a particular case and establish some estimates we will use throughout this work.

\subsection{Stokes problem}

Let $\Omega$ be a bounded open set in $\mathbb{R}^{m}(m=3)$ with smooth boundary $\partial \Omega=\Gamma$ and unit outward normal vector $n$.

The Stokes problem consists in finding a velocity vector $V$ of the fluid and a scalar function 
$q$ representing the pressure that satisfy the following equations and boundary condition.

$$
\begin{aligned}
& -\mu \Delta V+\nabla q=f \quad \text { in } \Omega, \\
& \operatorname{div} V=0 \quad \text { in } \Omega, \\
& V=0 \quad \text { on } \partial \Omega,
\end{aligned}
$$

where $\mu>0$ is the viscosity of the fluid.

Proposition 3.1 [19], [32].

If $f \in H^{s}(\Omega)$, then the problem (3.1)-(3.3) has a unique solution $(V, q)$ in the space $(\widetilde{\mathcal{V}} \cap$ $\left.H^{s+2}(\Omega)\right) \times\left(L_{0}^{2}(\Omega) \cap H^{s+1}(\Omega)\right)$, and the solution obeys the following estimate:

$$
\|V\|_{H^{s+2}}+\|q\|_{H^{s+1}} \leq C\|f\|_{H^{s}}, \quad C>0 .
$$

The following is another result related to the curl operator.

\section{Proposition 3.2 [9].}

Assume that the domain $\Omega$ is simply connected. Let $g$ be in $H^{s}(\Omega)$, then the function $g$ satisfies the condition $\operatorname{curl} g=0$ in $\Omega$ if and only if $g$ is a gradient of a unique function $q \in L_{0}^{2}(\Omega) \cap H^{s+1}(\Omega)$.

\subsection{Evolution problem}

Let $E$ be a separable Banach space, and $F$ a subspace embedded continuously and densely in $E$. For every $t \in[0, \mathrm{~T}]$ let $A(t)$ be a closed linear operator defined on $E$ with domain $D(A(t))$ and resolvent set $\rho(A(t))$. We assume that the family $\{A(t)\}_{t \in[0, T]}$ satisfies the following assumptions:

(i) There exist constants $M \geq 1$ and $\omega \in \mathbb{R}$ such that $\rho(A(t)) \supset(-\infty,-\omega)$ for all $t \in$ $[0, \mathrm{~T}]$ and

$$
\left\|\prod_{j=1}^{n}\left(\delta+A\left(t_{j}\right)\right)^{-n}\right\| \leq \frac{M}{(\delta-\omega)^{n}}, \quad \text { for } \delta>\omega
$$

and every finite sequence $\left\{t_{j}\right\}_{j=1}^{n}$ with $0 \leq t_{1}<t_{2}, \ldots, t_{n} \leq$ T for $n \in \mathbb{N}$. 
(ii) There is a family $\{S(t)\}_{t \in[0, \mathrm{~T}]}$ of isomorphisms of $F$ onto $E$ such that for every $p \in F$, the map $t \rightarrow S(t) p \in E$ is continuously differentiable on $[0, \mathrm{~T}]$ and

$$
S(t) A(t) S(t)^{-1}=A(t)+B(t), \quad t \in[0, \mathrm{~T}],
$$

where $B(t)$ is a linear bounded operator in $E$ and for each $p \in E$, the map $t \rightarrow B(t) p$ is continuous in $E$ on $[0, \mathrm{~T}]$.

(iii) The subspace $F$ is contained in $D(A(t)), A(t): F \rightarrow E$ is a bounded operator for all $t \in[0, \mathrm{~T}]$, and the mapping $t \rightarrow A(t) \in B(F, E)$ is norm continuous.

Consider the non-autonomous inhomogeneous initial value problem (or evolution problem)

$$
\begin{aligned}
& \frac{d}{d t} p(t)+A(t) p(t)=f(t) \quad t \in[0, \mathrm{~T}], \\
& p(0)=p_{0},
\end{aligned}
$$

where the function $f:[0, \mathrm{~T}] \longrightarrow E$.

Theorem 3.3 [24, Theorem 5.5.2 page 146]

Under the assumptions (i), (ii) and (iii) listed above, if $f \in C([0, T] ; F)$ then for every $p_{0} \in F$, there exists a unique solution

$$
p \in C([0, T] ; F) \cap C^{1}((0, T] ; E)
$$

of the initial value problem (3.6).

We will now consider the operator $A(t)$ defined by

$$
\begin{aligned}
A(t): H^{s}\left(\mathbb{R}^{3}\right) & \longrightarrow H^{s}\left(\mathbb{R}^{3}\right) \\
p & \longmapsto A(t) p=(U(t) \cdot \nabla) p+\lambda p
\end{aligned}
$$

where $U \in C\left([0, \mathrm{~T}] ; H^{s+2}\left(\mathbb{R}^{3}\right)\right)$ and $\lambda \in \mathbb{R}$. The domain $D(A(t))$ of the operator $A(t)$ contains $H^{s+1}\left(\mathbb{R}^{3}\right)$ which is dense in $H^{s}\left(\mathbb{R}^{3}\right)$. The operator $A(t)$ from $H^{s+1}\left(\mathbb{R}^{3}\right)$ into $H^{s}\left(\mathbb{R}^{3}\right)$ is bounded and the mapping $t \rightarrow A(t)$ is continuous in $B\left(H^{s+1}\left(\mathbb{R}^{3}\right), H^{s}\left(\mathbb{R}^{3}\right)\right)$ norm, since $t \rightarrow U(t) \in H^{s+2}\left(\mathbb{R}^{3}\right)$ is continuous. Hence the assumption (iii) is satisfied. 
Next, we show that the assumption $(i)$ is satisfied. Let $\delta$ be in $\mathbb{R}$, let $q$ be in $H^{s}\left(\mathbb{R}^{3}\right)$ and $p$ in $H^{s+1}\left(\mathbb{R}^{3}\right)$.

The equation $\delta p+A(t) p=q$ is equivalent to

$$
(\delta+\lambda) p+(U(t) \cdot \nabla) p=q
$$

Multiplying this equation by $p$ and integrating over $\mathbb{R}^{3}$, we have

$$
(\delta+\lambda) \int_{\mathbb{R}^{3}} p^{2} d \mathbf{x}+\frac{1}{2} \int_{\mathbb{R}^{3}}(U(t) \cdot \nabla) p^{2} d \mathbf{x}=\int_{\mathbb{R}^{3}} p q d \mathbf{x} .
$$

By divergence theorem and Sobolev embeddings

$$
\left|\int_{\mathbb{R}^{3}} U(t) \cdot \nabla p^{2} d \mathbf{x}\right|=\left|-\int_{\mathbb{R}^{3}} p^{2} \operatorname{div} U(t) d \mathbf{x},\right| \leq c_{s} \int_{\mathbb{R}^{3}} p^{2} d \mathbf{x}
$$

$c_{s}=\sup _{t \in[0, T]}\|U(t)\|_{H^{s+2}}$, then the equation (3.9) becomes after applying Cauchy-Schwarz inequality

$$
(\delta+\lambda)\|p\|_{L^{2}\left(\mathbb{R}^{3}\right)}^{2} \leq \frac{1}{2} c_{s}\|p\|_{L^{2}\left(\mathbb{R}^{3}\right)}^{2}+\|p\|_{L^{2}\left(\mathbb{R}^{3}\right)}\|q\|_{L^{2}\left(\mathbb{R}^{3}\right)} .
$$

Similarly, by differentiating (3.8) with respect to $\mathbf{x}^{\alpha},|\alpha| \leq s$ and using Leibniz formula we have

$$
(\delta+\lambda) D^{\alpha} p+U(t) \cdot \nabla\left(D^{\alpha} p\right)+\sum_{\mathbf{0}<\beta \leq \alpha}\left(\begin{array}{l}
\alpha \\
\beta
\end{array}\right) D^{\beta}(U(t)) \cdot \nabla\left(D^{\alpha-\beta} p\right)=D^{\alpha} q
$$

multiplying by $D^{\alpha} p$, then integrating over $\mathbb{R}^{3}$, we get

$$
\begin{aligned}
(\delta+\lambda) & \int_{\mathbb{R}^{3}}\left(D^{\alpha} p\right)^{2} d \mathbf{x}+\frac{1}{2} \int_{\mathbb{R}^{3}}(U(t) \cdot \nabla)\left(D^{\alpha} p\right)^{2} d \mathbf{x} \\
& +\sum_{\mathbf{0}<\beta \leq \alpha}\left(\begin{array}{c}
\alpha \\
\beta
\end{array}\right) \int_{\mathbb{R}^{3}} D^{\alpha} p D^{\beta} U(t) \cdot \nabla\left(D^{\alpha-\beta} p\right) d \mathbf{x}=\int_{\mathbb{R}^{3}} D^{\alpha} q D^{\alpha} p d \mathbf{x} .
\end{aligned}
$$

Again, by divergence theorem and Sobolev embeddings

$$
\left|\int_{\mathbb{R}^{3}}(U(t) \cdot \nabla)\left(D^{\alpha} p\right)^{2} d \mathbf{x}\right|=\left|-\int_{\mathbb{R}^{3}}\left(D^{\alpha} p\right)^{2} \operatorname{div} U(t) d \mathbf{x},\right| \leq c_{s}\left\|D^{\alpha} p\right\|_{L^{2}\left(\mathbb{R}^{3}\right)}^{2},
$$

and by Sobolev embeddings, there is a constant $k_{\alpha} \geq 0$ such that

$$
\left|\sum_{\mathbf{0}<\beta \leq \alpha}\left(\begin{array}{l}
\alpha \\
\beta
\end{array}\right) \int_{\mathbb{R}^{3}} D^{\alpha} p D^{\beta} U(t) \cdot \nabla\left(D^{\alpha-\beta} p\right) d \mathbf{x}\right| \leq c_{s} k_{\alpha}\|p\|_{H^{|\alpha|}\left(\mathbb{R}^{3}\right)}^{2} .
$$


Combining the inequalities (3.12) and (3.13) with the equality (3.11) and applying CauchySchwarz inequality we obtain

$$
\left(\delta+\lambda-\frac{1}{2} c_{s}\right)\left\|D^{\alpha} p\right\|_{L^{2}\left(\mathbb{R}^{3}\right)}^{2} \leq c_{s} k_{\alpha}\|p\|_{H^{|\alpha|}\left(\mathbb{R}^{3}\right)}^{2}+\left\|D^{\alpha} q\right\|_{L^{2}\left(\mathbb{R}^{3}\right)}\left\|D^{\alpha} p\right\|_{L^{2}\left(\mathbb{R}^{3}\right)} .
$$

Taking the sum over all $\alpha,|\alpha| \leq s$ we see that

$$
\|p\|_{H^{s}\left(\mathbb{R}^{3}\right)} \leq \frac{1}{\delta-\left(k_{s} c_{s}-\lambda\right)}\|q\|_{H^{s}\left(\mathbb{R}^{3}\right)}, \quad k_{s}>0
$$

which is equivalent to

$$
\left\|(\delta+A(t))^{-1} q\right\|_{H^{s}\left(\mathbb{R}^{3}\right)} \leq \frac{1}{\delta-\left(k_{s} c_{s}-\lambda\right)}\|q\|_{H^{s}\left(\mathbb{R}^{3}\right)} .
$$

It follows that the family $\{A(t)\}$ satisfies the assumption $(i)$ with $M=1$ and $\omega=k_{s} c_{s}-\lambda$. Now, we show that $(i i)$ is satisfied with the family of isomorphisms $S(t)=S=(I-\Delta)^{\frac{1}{2}}$ of $H^{s+1}\left(\mathbb{R}^{3}\right)$ onto $H^{s}\left(\mathbb{R}^{3}\right)$ and the operators, see [17, page 256$]$

$$
B(t)=(S U(t)-U(t) S) \cdot \nabla S^{-1}
$$

Note that $U(t)$ is the operator of multiplication by the function $U(t)$. The operator $S U(t)-$ $U(t) S$ is called the commutator of $S$ and $U(t)$, which can be extended to an operator defined on $H^{s}\left(\mathbb{R}^{3}\right)$ into $H^{s}\left(\mathbb{R}^{3}\right)$ [17]. This implies that $B(t)$ is a bounded operator on $H^{s}\left(\mathbb{R}^{3}\right)$ and for each $p \in H^{s}\left(\mathbb{R}^{3}\right)$, the map $t \rightarrow B(t) p$ is continuous in $H^{s}\left(\mathbb{R}^{3}\right)$ on $[0, \mathrm{~T}]$.

It remains to show that $S(t) A(t) S(t)^{-1}=A(t)+B(t)$ on $D(A(t)+B(t)) \equiv D(A(t))$. If $p \in H^{s+1}\left(\mathbb{R}^{3}\right)$, then since $\nabla$ and $S$ commute on $H^{s+2}\left(\mathbb{R}^{3}\right)$, we have

$$
\begin{aligned}
S(t) A(t) S(t)^{-1} p & =S((U(t) \cdot \nabla)+\lambda) S^{-1} p \\
& =A(t) p+S((U(t) \cdot \nabla)) S^{-1} p-U(t) \cdot(S \nabla) S^{-1} p \\
& =(A(t)+B(t)) p .
\end{aligned}
$$

Now, let $p \in D(A(t))$, there exists a sequence $\left(p_{n}\right)$ of functions in $H^{s+1}\left(\mathbb{R}^{3}\right)$ which converges to $p$ in $H^{s}\left(\mathbb{R}^{3}\right)$. Since $A(t)$ is closed,

$$
\begin{aligned}
A(t) S^{-1} p=\lim _{n \rightarrow \infty} A(t) S^{-1} p_{n} & =\lim _{n \rightarrow \infty} S^{-1}(A(t)+B(t)) p_{n} \\
& =S^{-1}(A(t)+B(t)) p \in H^{s+1}\left(\mathbb{R}^{3}\right) .
\end{aligned}
$$


Then, since $S: H^{s+1}\left(\mathbb{R}^{3}\right) \rightarrow H^{s}\left(\mathbb{R}^{3}\right)$ is an isomorphism, we have

$$
S A(t) S^{-1} p=(A(t)+B(t)) p
$$

Hence the assumption (ii) holds, so we could apply Theorem 3.3 to conclude the following proposition.

Proposition 3.4 For each $p_{0} \in H^{s+1}\left(\mathbb{R}^{3}\right)$, the problem (3.6) with $A(t)$ defined in (3.7) has a unique solution

$$
p \in C\left([0, T] ; H^{s+1}\left(\mathbb{R}^{3}\right)\right) \cap C^{1}\left((0, T] ; H^{s}\left(\mathbb{R}^{3}\right)\right)
$$

Next, we extend this result to the bounded domain $\Omega$ with smooth boundary $\partial \Omega$ by using the extension theorem (Theorem 2.6).

Let $V(t) \in\left(H^{s+2}(\Omega)\right)^{3}, \lambda \in \mathbb{R}$ and consider the problem

$$
\begin{aligned}
& \frac{d}{d t} p(t)+(V(t) \cdot \nabla+\lambda) p(t)=f(t) \quad t \in[0, \mathrm{~T}] \\
& p(0)=p_{0} .
\end{aligned}
$$

As a consequence of the above results, we have the following proposition.

Proposition 3.5 For every $p_{0} \in H^{s+1}(\Omega)$, there exists a solution

$$
p \in C\left([0, T] ; H^{s+1}(\Omega)\right) \cap C^{1}\left((0, T] ; H^{s}(\Omega)\right)
$$

of the initial value problem (3.15).

Proof. By Theorem 2.6 we can extend $p_{0}$ to $p_{0 e} \in H^{s+1}\left(\mathbb{R}^{3}\right), f(t)$ to $f_{e}(t) \in H^{s}\left(\mathbb{R}^{3}\right)$ and $V(t)$ to $U(t) \in H^{s+2}\left(\mathbb{R}^{3}\right)$. Then, applying Proposition 3.4, there exists a solution $p_{e} \in C\left([0, \mathrm{~T}] ; H^{s+1}\left(\mathbb{R}^{3}\right)\right) \cap C^{1}\left((0, \mathrm{~T}] ; H^{s}\left(\mathbb{R}^{3}\right)\right)$ of the problem (3.6) with extended operator $(U(t) \cdot \nabla)+\lambda$ and extended functions $p_{0 e}$ and $f_{e}(t)$. Since the restriction of an extension of a function is the function itself, then the restriction of $p_{e}$ to $\Omega$ is a solution for the problem (3.15).

Proposition 3.5 can be also obtained from the results of [4]. 
Remark 3.6 Because of non-uniqueness of extended function, we need the assumption that the normal component is vanishing on the boundary, to guarantee the uniqueness.

Before proving uniqueness, we need the following lemmas.

Lemma 3.7 Case $T<\infty, \lambda \in \mathbb{R}$ :

Let $f \in C\left([0, T] ; H^{s}(\Omega)\right)$ and $p \in C\left([0, T] ; H^{s}(\Omega)\right)$ satisfying the differential inequality

$$
\frac{d}{d t}\|p(t)\|_{H^{s}}+\lambda\|p(t)\|_{H^{s}} \leq\|f(t)\|_{H^{s}}
$$

with $p(0)=p_{0}$. Then the following estimate holds:

$$
\|p\|_{C\left([0, T] ; H^{s}\right)} \leq C_{1}(\lambda, T)\left\|p_{0}\right\|_{H^{s}}+C_{2}(\lambda, T)\|f\|_{C\left([0, T] ; H^{s}\right)},
$$

where $C_{1}(\lambda, T)=\max _{0 \leq t \leq T} e^{-\lambda t}, C_{2}(\lambda, T)=\frac{1-e^{-\lambda T}}{\lambda}$.

Proof. We rewrite the differential inequality (3.16) as

$$
\frac{d}{d t}\left(e^{\lambda t}\|p(t)\|_{H^{s}}\right) \leq e^{\lambda t}\|f(t)\|_{H^{s}}
$$

then integrate with respect to $t$ over the interval $[0, t], t \leq \mathrm{T}$. We obtain the following inequality:

$$
\|p(t)\|_{H^{s}} \leq e^{-\lambda t}\left\|p_{0}\right\|_{H^{s}}+\int_{0}^{t} e^{\lambda(\tau-t)}\|f(\tau)\|_{H^{s}} d \tau
$$

Next, we take the supremum over the time interval $[0, \mathrm{~T}]$

$$
\|p\|_{C\left([0, T] ; H^{s}\right)} \leq C_{1}(\lambda, \mathrm{T})\left\|p_{0}\right\|_{H^{s}}+C_{2}(\lambda, \mathrm{T})\|f\|_{C\left([0, \mathrm{~T}] ; H^{s}\right)},
$$

where $C_{1}(\lambda, \mathrm{T})=\max _{0 \leq t \leq \mathrm{T}} e^{-\lambda t}$ and $C_{2}(\lambda, \mathrm{T})=\frac{1-e^{-\lambda \mathrm{T}}}{\lambda}$.

Lemma 3.8 Case $T=\infty, \lambda>0$ :

Let $f \in C_{b}\left([0, \infty) ; H^{s}(\Omega)\right)$ and let $p \in C\left([0, \infty) ; H^{s}(\Omega)\right)$ satisfies

$$
\frac{d}{d t}\|p(t)\|_{H^{s}}+\lambda\|p(t)\|_{H^{s}} \leq\|f(t)\|_{H^{s}}, \quad p(0)=p_{0} .
$$

Then, we have

$$
\|p\|_{C_{b}\left([0, \infty) ; H^{s}\right)} \leq\left\|p_{0}\right\|_{H^{s}}+\frac{1}{\lambda}\|f\|_{C_{b}\left([0, \infty) ; H^{s}\right)}
$$


Proof. The proof of this lemma is similar to the case $\mathrm{T}<\infty, \lambda \in \mathbb{R}$. (see Lemma 3.7).

Next, we establish the estimates for the problem (3.15).

Lemma 3.9 Let $p_{0} \in H^{s+1}(\Omega), f \in C_{b}\left([0, T) ; H^{s+1}(\Omega)\right)$ and assume that $\operatorname{div} V(t)=0$ in $\Omega, V(t) \equiv 0$ on $\partial \Omega$. Then the following estimates hold for any solution $p$ of the problem (3.15).

For $T<\infty, \lambda \in \mathbb{R}$ and $\|V\|_{C_{b}\left([0, T] ; H^{s+2}\right)} \leq \varepsilon$ for an arbitrary $\varepsilon>0$ we have

$$
\|p\|_{C_{b}\left([0, T] ; H^{s+1}\right)} \leq C_{1}(\lambda, T)\left\|p_{0}\right\|_{H^{s+1}}+C_{2}(\lambda, T)\|f\|_{C_{b}\left([0, T] ; H^{s+1}\right)},
$$

where $C_{1}(\lambda, T)=\max _{0 \leq t \leq T} e^{-\left(\lambda-C_{s} \varepsilon\right) t}, C_{2}(\lambda, T)=\frac{1-e^{-\left(\lambda-C_{s} \varepsilon\right) T}}{\lambda-C_{s} \varepsilon}, C_{s}>0$.

For $T=\infty, \lambda>0$ and $\|V\|_{C_{b}\left([0, \infty) ; H^{s+2}\right)} \leq \varepsilon$ with $\varepsilon$ sufficiently small:

$$
\|p\|_{C_{b}\left([0, \infty) ; H^{s+1}\right)} \leq\left\|p_{0}\right\|_{H^{s+1}}+\frac{1}{\lambda-C_{s} \varepsilon}\|f\|_{C_{b}\left([0, \infty) ; H^{s+1}\right)}, C_{s}>0 .
$$

Proof. Multiplying the first equation of the problem (3.15) by $p(t)$, and integrating over the domain $\Omega$, we get

$$
\frac{1}{2} \frac{d}{d t} \int_{\Omega}(p(t))^{2} d \mathbf{x}+\frac{1}{2} \int_{\Omega}(V(t) \cdot \nabla)(p(t))^{2} d \mathbf{x}+\lambda \int_{\Omega}(p(t))^{2} d \mathbf{x}=\int_{\Omega} f(t) p(t) d \mathbf{x} .
$$

Since $\operatorname{div} V(t)=0$ in $\Omega \times[0, \mathrm{~T}]$ and $V(t)=0$ on $\partial \Omega$, then applying the divergence theorem we see that

$$
\int_{\Omega}(V(t) \cdot \nabla)(p(t))^{2} d \mathbf{x}=0
$$

We use Cauchy-Schwarz inequality to estimate the right hand side of (3.24), then we get

$$
\frac{1}{2} \frac{d}{d t}\|p(t)\|_{L^{2}}^{2}+\lambda\|p(t)\|_{L^{2}}^{2} \leq\|f(t)\|_{L^{2}}\|p(t)\|_{L^{2}} .
$$

Next, if we differentiate with respect to $\mathbf{x}^{\alpha},|\alpha| \leq s+1$ the first equation of (3.15) and use Leibniz formula to rewrite $D^{\alpha}[(V(t) \cdot \nabla) p(t)]$, then multiply the obtained equation by $D^{\alpha} p(t)$ and integrate over $\Omega$, we obtain

$$
\begin{aligned}
\frac{1}{2} \frac{d}{d t} \int_{\Omega}\left(D^{\alpha} p(t)\right)^{2} d \mathbf{x}+\frac{1}{2} \int_{\Omega}(V(t) \cdot \nabla)\left(D^{\alpha} p(t)\right)^{2} d \mathbf{x}+\lambda \int_{\Omega}\left(D^{\alpha} p(t)\right)^{2} d \mathbf{x}+ \\
\sum_{\mathbf{0}<\beta \leq \alpha}\left(\begin{array}{c}
\alpha \\
\beta
\end{array}\right) \int_{\Omega} D^{\alpha} p(t) D^{\beta} V(t) \cdot \nabla\left(D^{\alpha-\beta} p(t)\right) d \mathbf{x}=\int_{\Omega} D^{\alpha} f(t) D^{\alpha} p(t) d \mathbf{x} .
\end{aligned}
$$


Applying divergence theorem and using the fact that $\operatorname{div} V(t)=0$ in $\Omega \times[0, \mathrm{~T}]$ and $V(t)=0$ on $\partial \Omega \times[0, \mathrm{~T}]$, we get

$$
\int_{\Omega}(V(t) \cdot \nabla)\left(D^{\alpha} p(t)\right)^{2}=0
$$

By Sobolev embedding theorem, there exists $C_{\alpha}>0$ such that

$$
\left|\sum_{\mathbf{0}<\beta \leq \alpha}\left(\begin{array}{c}
\alpha \\
\beta
\end{array}\right) \int_{\Omega} D^{\alpha} p(t) D^{\beta} V(t) \cdot \nabla\left(D^{\alpha-\beta} p(t)\right)\right| \leq C_{\alpha}\|V(t)\|_{H^{s+2}}\|p\|_{H^{|\alpha|}}^{2}
$$

and by Cauchy-Schwarz inequality

$$
\left|\int_{\Omega} D^{\alpha} f(t) D^{\alpha} p(t) d \mathbf{x}\right| \leq\left\|D^{\alpha} f(t)\right\|_{L^{2}}\left\|D^{\alpha} p(t)\right\|_{L^{2}} .
$$

With the above estimates, the equation (3.26) gives

$$
\begin{aligned}
& \frac{1}{2} \frac{d}{d t}\left\|D^{\alpha} p(t)\right\|_{L^{2}}^{2}+\lambda\left\|D^{\alpha} p(t)\right\|_{L^{2}}^{2} \leq \\
& C_{\alpha}\|V(t)\|_{H^{s+2}}\|p\|_{H^{|\alpha|}}^{2}+\left\|D^{\alpha} f(t)\right\|_{L^{2}}\left\|D^{\alpha} p(t)\right\|_{L^{2}} .
\end{aligned}
$$

We take the sum over all $\alpha \leq s+1$ and noting that

$$
\frac{1}{2} \frac{d}{d t}\|p(t)\|_{H^{s+1}}^{2}=\|p(t)\|_{H^{s+1}} \frac{d}{d t}\|p(t)\|_{H^{s+1}}
$$

we obtain

$$
\frac{d}{d t}\|p(t)\|_{H^{s+1}}+\lambda\|p(t)\|_{H^{s+1}} \leq C_{s}\|V(t)\|_{H^{s+2}}\|p(t)\|_{H^{s+1}}+\|f(t)\|_{H^{s+1}} .
$$

We now apply the Lemmas 3.7 and 3.8 to establish the estimates (3.22) and (3.23).

Corollary 3.10 If $\operatorname{div} V(t)=0$ in $\Omega$ and $V(t) \equiv 0$ on $\partial \Omega$ with $\|V(t)\|_{H^{s+2}} \leq \varepsilon$, then for every $p_{0} \in H^{s+1}(\Omega)$, the evolution problem (3.15) has a unique solution $p$.

If $T<\infty, \lambda \in \mathbb{R}$ and $\varepsilon$ arbitrary $>0$, then

$$
p \in C\left([0, T] ; H^{s+1}(\Omega)\right) \cap C^{1}\left((0, T] ; H^{s}(\Omega)\right)
$$

and satisfies the estimate (3.22).

If $T=\infty, \lambda>0$ and $\varepsilon$ sufficiently small, then

$$
p \in C_{b}\left([0, \infty) ; H^{s+1}(\Omega)\right) \cap C^{1}\left((0, \infty) ; H^{s}(\Omega)\right)
$$

and satisfies the estimate (3.23). 
Proof. Let $p_{1}$ and $p_{2}$ be two solutions of (3.15) with $p_{1}(0)=p_{2}(0)=p_{0}$, then $p_{1}-p_{2}$ is a solution of the homogeneous problem with initial value $\left(p_{1}-p_{2}\right)(0)=0$. Hence the estimates (3.22) and (3.23) can ensure the uniqueness of the solution. 


\section{$\mathrm{C}^{\mathrm{h}^{\mathrm{p}} \mathrm{p}_{\mathrm{r}}} \mathrm{H}$}

\section{Three-dimensional flow with small initial data}

\section{Contents}

4.1 Preliminaries and main result . . . . . . . . . . . . . . 39

4.2 Reduction to a sequence of Stokes and evolution problems . 40

4.3 Construction of the sequence $\left(V^{n}, q^{n}, p^{n}, \mathbf{T}^{n}\right) \ldots \ldots 43$

$4.3 .1 \quad$ Existence and uniqueness . . . . . . . . . . . . . . . 43

4.3 .2 Convergence . . . . . . . . . . . . . . . . . 46

In this chapter, we prove the existence and uniqueness of solutions for the problem of the equations (1.12)-(1.16) with sufficiently small initial data, which physically represents a three-dimensional problem of an unsteady flow of a quasistatic viscoelastic fluid. The proof is based on an iterative method introduced by Renardy [26], [29]. 


\subsection{Preliminaries and main result}

Let $\Omega$ be a bounded domain in $\mathbb{R}^{3}$ with smooth boundary $\partial \Omega$ and unit outward normal vector $n$. Let $\mathrm{T}$ be in the interval $(0, \infty]$. We consider the problem of the equations (1.12)-(1.16), describing an unsteady flow of a quasistatic viscoelastic fluid in three-dimensional geometry. Recall that the body force $\mathbf{f}$ and the initial extra stress tensor $\mathbf{T}_{0}$ are given and assumed to be sufficiently small in their appropriate norms. The unknowns are: The velocity vector field $V=\left(V_{1}, V_{2}, V_{3}\right)$, the pressure force $p$, and the extra stress tensor $\mathbf{T}=\left(T_{i j}\right)_{1 \leq i, j \leq 3}$. The mapping $g$ in the left hand side of equation (1.15),

$$
\begin{aligned}
g: \mathbb{R}^{3 \times 3} \times \mathbb{R}^{3 \times 3} & \longrightarrow \mathbb{R}^{3 \times 3} \\
(M, N) & \longmapsto g(M, N),
\end{aligned}
$$

is a tensor valued function of second-order tensors and is assumed to be of class $C^{s+3}$ on $\mathbb{R}^{3 \times 3} \times \mathbb{R}^{3 \times 3}$ with $g$ and its gradient with respect to $(M, N)$ vanish at $(0,0)$, with constants $\lambda \in \mathbb{R}$ and $\mu>0$. Where $s$ is an integer $\geq 1$ so that by Sobolev embedding theorem we have $H^{s+1}(\Omega) \hookrightarrow C_{b}^{s-1}(\bar{\Omega})$ and $\prod_{i=1}^{r} D^{\alpha_{i}} u_{i} \in L^{2}(\Omega), \prod_{i=1}^{r} u_{i} \in H^{s+1}(\Omega)$ for every $\left(u_{i}\right)_{1 \leq i \leq r}$ in $H^{s+1}(\Omega)$ with $\sum_{i=1}^{r}\left|\alpha_{i}\right| \leq s+1$, see [35, Corollary 21.78 page 295] and [1, Theorem 5.23 page $115]$.

Proposition 4.1 Under the previous assumptions, the function $g$ and its derivatives up to order 2 map $H^{s+1}(\Omega) \times H^{s+1}(\Omega)$ into $H^{s+1}(\Omega)$ and the composite function $g(\nabla V, \mathbf{T})$ satisfies a local Lipschitz condition of order 2 at $(\nabla V, \mathbf{T})=(0,0)$, in the sense that for given $c>0$, we have

$$
\|g(\nabla V, \mathbf{T})\|_{H^{s+1}} \leq L\left(\|V\|_{H^{s+2}}^{2}+\|\mathbf{T}\|_{H^{s+1}}^{2}\right)
$$

for all $(V, \mathbf{T})$ in $H^{s+2}(\Omega) \times H^{s+1}(\Omega)$ with $\|V\|_{H^{s+2}} \leq c$ and $\|\mathbf{T}\|_{H^{s+1}} \leq c$. The constant $L=L(c)>0$.

Proof. The proof is an application of Corollary 2.10.

The main result of this section is the following theorem. 
Theorem 4.2 Let $s$ be an integer $\geq 1$. Suppose there is $\varepsilon>0$ sufficiently small such that

$$
\left\|\mathbf{T}_{0}\right\|_{H^{s+1}}+\|\mathbf{f}\|_{C_{b}\left([0, T) ; H^{s+1}\right)}+\left\|\frac{\partial}{\partial t} \mathbf{f}\right\|_{C_{b}\left([0, T) ; H^{s}\right)} \leq \varepsilon,
$$

subject to the constraint that $\operatorname{div}\left(\mathbf{T}_{0}\right)-\mathbf{f}(\cdot, 0)$ is a gradient. Then there exists a unique triplet $(V, p, \mathbf{T})$, belonging to the space

$$
C_{b}\left([0, T) ; H^{s+2}(\Omega)\right) \times C_{b}\left([0, T) ; H^{s+1}(\Omega) \cap L_{0}^{2}(\Omega)\right) \times C_{b}\left([0, T) ; H^{s+1}(\Omega)\right),
$$

solution to problem (1.12)-(1.16).

In what follows, we assume that there exists $\varepsilon>0$ sufficiently small such that the estimate (4.2) is satisfied.

\subsection{Reduction to a sequence of Stokes and evolution problems}

A solution of the problem (1.12)-(1.16) can be obtained by constructing a sequence of three subproblems whose solutions converge to a solution of the problem (1.12)-(1.16). At each iteration we need to solve a Stokes problem and two evolution problems. This method is introduced by Renardy in [26], [27], [29] for steady flow problems. The basic idea of this method consists of taking the divergence operator on both sides of the constitutive equation $(1.15)$

$$
\begin{aligned}
\frac{\partial}{\partial t} \operatorname{div} \mathbf{T}+(V \cdot \nabla) \operatorname{div} \mathbf{T} & +\sum_{j=1}^{3}\left(\frac{\partial V}{\partial x_{j}} \cdot \nabla\right) \mathbf{T}_{i j}+\lambda \operatorname{div} \mathbf{T} \\
& =\mu(\Delta V+\nabla(\operatorname{div} V))+\operatorname{div}(g(\nabla V, \mathbf{T})) .
\end{aligned}
$$

From the equation (1.13) $\operatorname{div} V=0$, and from the equation (1.12), we can substitute $\operatorname{div} \mathbf{T}$ by $\nabla p+\mathbf{f}$

$$
\begin{aligned}
\frac{\partial}{\partial t} \nabla p+(V & \cdot \nabla) \nabla p+\lambda \nabla p+\sum_{j=1}^{3}\left(\frac{\partial V}{\partial x_{j}} \cdot \nabla\right) \mathbf{T}_{i j} \\
& =\mu \Delta V+\operatorname{div}(g(\nabla V, \mathbf{T}))-\frac{\partial \mathbf{f}}{\partial t}-(V \cdot \nabla) \mathbf{f}-\lambda \mathbf{f} .
\end{aligned}
$$


Using the properties of nabla operator, $(V \cdot \nabla) \nabla p$ can be rewritten as

$$
(V \cdot \nabla) \nabla p=\nabla((V \cdot \nabla) p)-(\nabla V)^{\mathrm{T}} \nabla p
$$

Substituting in (4.4) we have

$$
\begin{aligned}
\nabla\left(\frac{\partial}{\partial t}+V \cdot \nabla+\lambda\right) & p-(\nabla V)^{\mathrm{T}} \nabla p+\sum_{j=1}^{3}\left(\frac{\partial V}{\partial x_{j}} \cdot \nabla\right) \mathbf{T}_{i j} \\
& =\mu \Delta V+\operatorname{div}(g(\nabla V, \mathbf{T}))-\frac{\partial \mathbf{f}}{\partial t}-(V \cdot \nabla) \mathbf{f}-\lambda \mathbf{f} .
\end{aligned}
$$

We now introduce a new function $q$,

$$
q=\left(\frac{\partial}{\partial t}+V \cdot \nabla+\lambda\right) p
$$

Using this new function, the problem (1.12)-(1.16) can be reformulated as follows: Find a velocity vector field $V$, an extra stress tensor $\mathbf{T}$, a scalar pressure $p$ and a scalar function $q$ satisfying the equations

$$
\begin{aligned}
& q=\left(\frac{\partial}{\partial t}+V \cdot \nabla+\lambda\right) p \quad \text { in } \Omega \times[0, \mathrm{~T}), \\
& -\mu \Delta V+\nabla q=-\sum_{j=1}^{3}\left(\frac{\partial V}{\partial x_{j}} \cdot \nabla\right) \mathbf{T}_{i j}+(\nabla V)^{\mathrm{T}} \nabla p \\
& +\operatorname{div}(g(\nabla V, \mathbf{T}))-\frac{\partial \mathbf{f}}{\partial t}-(V \cdot \nabla) \mathbf{f}-\lambda \mathbf{f} \text { in } \Omega \times[0, \mathrm{~T}), \\
& V=0 \quad \text { on } \partial \Omega \times[0, \mathrm{~T}), \\
& \frac{\partial \mathbf{T}}{\partial t}+(V \cdot \nabla) \mathbf{T}+\lambda \mathbf{T}=\mu\left(\nabla V+(\nabla V)^{\mathrm{T}}\right)+g(\nabla V, \mathbf{T}) \text { in } \Omega \times[0, \mathrm{~T}), \\
& \mathbf{T}(\cdot, 0)=\mathbf{T}_{0} \quad \text { in } \Omega .
\end{aligned}
$$

We note that by Proposition 3.2, the constraint condition (1.17) guarantees the existence of a unique $p_{0}(\mathbf{x}) \in L_{0}^{2}(\Omega) \cap H^{s+1}(\Omega)$, satisfying the equation (1.12) in $\Omega$ at $t=0$,

$$
\nabla p_{0}=\operatorname{div} \mathbf{T}_{0}-\mathbf{f}(\cdot, 0)
$$


We now construct the sequence of the three subproblems we mentioned in the beginning of this section. As in numerical iterative methods, to initialize a scheme we need an initial guess for our expected solution. It is obvious to start with the boundary condition value for $V$ and initial values for $\mathbf{T}$ and $p$.

$$
V^{0}(\mathbf{x}, t)=0, \mathbf{T}^{0}(\mathbf{x}, t)=\mathbf{T}_{0}(\mathbf{x}), p^{0}(\mathbf{x}, t)=p_{0}(\mathbf{x})
$$

In the next iteration, we solve the following Stokes problem for the vector field $V^{n+1}$ and the scalar $q^{n+1}$

$$
\begin{array}{ll}
-\mu \Delta V^{n+1}+\nabla q^{n+1}= & -\sum_{j=1}^{3}\left(\frac{\partial V^{n}}{\partial x_{j}} \cdot \nabla\right) \mathbf{T}_{i j}^{n}+\left(\nabla V^{n}\right)^{\mathrm{T}} \nabla p^{n}+\operatorname{div}\left(g\left(\nabla V^{n}, \mathbf{T}^{n}\right)\right) \\
\operatorname{div} V^{n+1}=0 & -\frac{\partial \mathbf{f}}{\partial t}-\left(V^{n} \cdot \nabla\right) \mathbf{f}-\lambda \mathbf{f} \text { in } \Omega \times[0, \mathrm{~T}), \\
V^{n+1}=0 & \text { in } \Omega \times[0, \mathrm{~T}), \\
& \text { on } \partial \Omega \times[0, \mathrm{~T}) .
\end{array}
$$

We assume that the solution of this problem is already known. To determine $\mathbf{T}^{n+1}$ and $p^{n+1}$, we solve the following evolution problem for $p^{n+1}$ :

$$
\left.\begin{array}{ll}
\frac{\partial}{\partial t} p^{n+1}+\left(V^{n+1} \cdot \nabla\right) p^{n+1}+\lambda p^{n+1}=q^{n+1} & \text { in } \Omega \times[0, \mathrm{~T}), \\
p^{n+1}(\cdot, 0)=p_{0} & \text { in } \Omega,
\end{array}\right\}
$$

and a similar problem for $\mathbf{T}^{n+1}$ :

$$
\begin{aligned}
& \begin{array}{ll}
\frac{\partial \mathbf{T}^{n+1}}{\partial t}+\left(V^{n+1} \cdot \nabla\right) \mathbf{T}^{n+1}+\lambda \mathbf{T}^{n+1}=\mu & \left(\nabla V^{n+1}+\left(\nabla V^{n+1}\right)^{\mathrm{T}}\right) \\
& +g\left(\nabla V^{n+1}, \mathbf{T}^{n}\right) \quad \text { in } \Omega \times[0, \mathrm{~T}),
\end{array} \\
& \mathbf{T}^{n+1}(\cdot, 0)=\mathbf{T}_{0} \quad \text { in } \Omega .
\end{aligned}
$$

If we assume that the prior two problems (4.17) and (4.18) have solutions, then we continue the process to construct a sequence $\left(V^{n}, q^{n}, p^{n}, \mathbf{T}^{n}\right)$. The goal of the next section is to prove the existence and the convergence of this sequence to a solution of the problem (4.8)-(4.13) in suitable spaces of functions. 


\subsection{Construction of the sequence $\left(V^{n}, q^{n}, p^{n}, \mathbf{T}^{n}\right)$}

\subsubsection{Existence and uniqueness}

We prove the existence and uniqueness of the solutions for problems (4.16), (4.17) and (4.18) in the following lemmas.

Lemma 4.3 Let $T \in(0, \infty]$. If $V^{n} \in C_{b}\left([0, T) ; H^{s+2}(\Omega)\right), p^{n} \in C_{b}\left([0, T) ; H^{s+1}(\Omega)\right)$ and $\mathbf{T}^{n} \in C_{b}\left([0, T) ; H^{s+1}(\Omega)\right)$, then the problem (4.16) has a unique solution

$$
\left(V^{n+1}, q^{n+1}\right) \in C_{b}\left([0, T) ; H^{s+2}(\Omega)\right) \times C_{b}\left([0, T) ; H^{s+1}(\Omega) \cap L_{0}^{2}(\Omega)\right) .
$$

Moreover, there exist $\varepsilon_{1}>0$ and $\varepsilon_{2}>0$ such that the following stability condition holds if $\left\|V^{n}\right\|_{C_{b}\left([0, T) ; H^{s+2}\right)} \leq \varepsilon_{1}$ and $\left\|\mathbf{T}^{n}\right\|_{C_{b}\left([0, T) ; H^{s+1}\right)} \leq \varepsilon_{2}$ :

$$
\begin{aligned}
& \left\|V^{n+1}\right\|_{C_{b}\left([0, T) ; H^{s+2}\right)}+\left\|q^{n+1}\right\|_{C_{b}\left([0, T) ; H^{s+1}\right)} \leq \\
& C_{1}\left(\left\|V^{n}\right\|_{C_{b}\left([0, T) ; H^{s+2}\right)}^{2}+\left\|p^{n}\right\|_{C_{b}\left([0, T) ; H^{s+1}\right)}^{2}+\left\|\mathbf{T}^{n}\right\|_{C_{b}\left([0, T) ; H^{s+1}\right)}^{2}+\varepsilon\right),
\end{aligned}
$$

where $C_{1}$ is a non-negative constant independent of $n$ and $\varepsilon$ is the bound defined in the inequality (4.2).

Proof. Existence and uniqueness follow from Proposition 3.1. We note that the solution of the Stokes problem depends continuously on $t$ if the right hand side of the equation does. Moreover, the following estimate holds for some constant $C$ independent of $n$ and $t$ :

$$
\left\|V^{n+1}\right\|_{H^{s+2}}+\left\|q^{n+1}\right\|_{H^{s+1}} \leq C\left\|\mathcal{M}\left(V^{n}, p^{n}, \mathbf{T}^{n}, \mathbf{f}\right)\right\|_{H^{s}}
$$

where $\mathcal{M}\left(V^{n}, p^{n}, \mathbf{T}^{n}, \mathbf{f}\right)$ denotes the right hand side of the first equation of (4.16).

By Sobolev embedding theorem, there exists $C_{0}>0$ such that

$$
\begin{aligned}
& \left\|-\sum_{j=1}^{3}\left(\frac{\partial V^{n}}{\partial x_{j}} \cdot \nabla\right) \mathbf{T}_{i j}^{n}\right\|_{H^{s}} \leq C_{0}\left\|V^{n}\right\|_{H^{s+2}}\left\|\mathbf{T}^{n}\right\|_{H^{s+1}}, \\
& \left\|\left(\nabla V^{n}\right)^{\mathrm{T}} \nabla p^{n}\right\|_{H^{s}} \leq C_{0}\left\|V^{n}\right\|_{H^{s+2}}\left\|p^{n}\right\|_{H^{s+1}} .
\end{aligned}
$$


Using the inequality $\left\|\operatorname{div}\left(g\left(\nabla V^{n}, \mathbf{T}^{n}\right)\right)\right\|_{H^{s}} \leq\left\|g\left(\nabla V^{n}, \mathbf{T}^{n}\right)\right\|_{H^{s+1}}$ and the fact that $g$ satisfies the condition (4.1), we shall get

$$
\left\|\operatorname{div}\left(g\left(\nabla V^{n}, \mathbf{T}^{n}\right)\right)\right\|_{H^{s}} \leq C\left(\left\|V^{n}\right\|_{H^{s+2}}^{2}+\left\|\mathbf{T}^{n}\right\|_{H^{s+1}}^{2}\right),
$$

where the constant $C$ is independent of $n$ whenever

$$
\left\|V^{n}\right\|_{H^{s+2}} \leq \varepsilon_{1} \text { and }\left\|\mathbf{T}^{n}\right\|_{H^{s+1}} \leq \varepsilon_{2} .
$$

Finally,

$$
\left\|\frac{\partial}{\partial t} \mathbf{f}+\left(V^{n} \cdot \nabla\right) \mathbf{f}+\lambda \mathbf{f}\right\|_{H^{s}} \leq C \varepsilon .
$$

Combining the inequalities (4.20), (4.21), (4.22), (4.23) and (4.24), we have the following estimate:

$$
\left\|V^{n+1}\right\|_{H^{s+2}}+\left\|q^{n+1}\right\|_{H^{s+1}} \leq C_{1}\left(\left\|V^{n}\right\|_{H^{s+2}}^{2}+\left\|p^{n}\right\|_{H^{s+1}}^{2}+\left\|\mathbf{T}^{n}\right\|_{H^{s+1}}^{2}+\varepsilon\right) .
$$

Then we take the supremum over $t$ to obtain the inequality required.

Lemma 4.4 Let $T$ be in $(0, \infty]$. Assume that $p_{0} \in H^{s+1}(\Omega), q^{n+1} \in C_{b}\left([0, T) ; H^{s+1}(\Omega)\right)$ and $V^{n+1} \in C_{b}\left([0, T) ; H^{s+2}(\Omega)\right)$ with $\operatorname{div} V^{n+1}=0$ in $\Omega \times[0, T), V^{n+1}=0$ on $\partial \Omega \times[0, T)$ and $\left\|V^{n+1}\right\|_{C_{b}\left([0, T) ; H^{s+2}\right)} \leq \varepsilon_{1}$ for a sufficiently small $\varepsilon_{1}>0$. Then there exists a unique $p^{n+1} \in C_{b}\left([0, T) ; H^{s+1}(\Omega)\right)$ satisfying the problem (4.17) and the following estimate holds:

$$
\left\|p^{n+1}\right\|_{C_{b}\left([0, T) ; H^{s+1}\right)} \leq C_{2}\left(\left\|p_{0}\right\|_{H^{s+1}}+\left\|q^{n+1}\right\|_{C_{b}\left([0, T) ; H^{s+1}\right)}\right) .
$$

The constant $C_{2}>0$ is independent of $n$.

Proof. Existence and uniqueness of $p^{n+1}$ are an immediate consequence of Proposition 3.5. To show the estimate (4.26), just apply Lemma 3.9 .

Recall that the function $g$ is assumed to satisfy a local Lipschitz condition in $(\nabla V, \mathbf{T})$ of order 2, see inequality (4.1).

Lemma 4.5 Let $T \in(0, \infty]$. Assume that $V^{n+1}$ is in the space $C_{b}\left([0, T) ; H^{s+2}(\Omega)\right)$, $\mathbf{T}^{n}$ in $C_{b}\left([0, T) ; H^{s+1}(\Omega)\right)$ with $\operatorname{div} V^{n+1}(t)=0$ in $\Omega, V^{n+1}(t)=0$ on $\partial \Omega$ and $\left\|V^{n+1}\right\|_{C_{b}\left([0, T) ; H^{s+2}\right)} \leq$ 
$\varepsilon_{1},\left\|\mathbf{T}^{n}\right\|_{C_{b}\left([0, T) ; H^{s+1}\right)} \leq \varepsilon_{2}$ for $\varepsilon_{1}, \varepsilon_{2}$ sufficiently small. Then there exists a unique solution $\mathbf{T}^{n+1} \in C_{b}\left([0, T) ; H^{s+1}(\Omega)\right)$ of the problem (4.18).

Furthermore, the following stability condition holds:

$$
\left\|\mathbf{T}^{n+1}\right\|_{C_{b}\left([0, T) ; H^{s+1}\right)} \leq C_{3}\left(\left\|\mathbf{T}_{0}\right\|_{H^{s+1}}+\left\|V^{n+1}\right\|_{C_{b}\left([0, T) ; H^{s+2}\right)}+\left\|\mathbf{T}^{n}\right\|_{C_{b}\left([0, T) ; H^{s+1}\right)}^{2}\right),
$$

where $C_{3}$ is a non-negative constant independent of $n$.

Proof. The proof of existence and uniqueness is an application of Proposition 3.5. It remains to establish the estimate (4.27). Using Lemma 3.9, we have

$$
\begin{aligned}
& \left\|\mathbf{T}^{n+1}\right\|_{C_{b}\left([0, \mathrm{~T}) ; H^{s+1}\right)} \leq C\left\|\mathbf{T}_{0}\right\|_{H^{s+1}}+ \\
& \quad C\left\|\mu\left(\nabla V^{n+1}+\left(\nabla V^{n+1}\right)^{\mathrm{T}}\right)+g\left(\nabla V^{n+1}, \mathbf{T}^{n}\right)\right\|_{C_{b}\left([0, \mathrm{~T}) ; H^{s+1}\right)}
\end{aligned}
$$

Then, we use the fact that $g$ satisfies the local Lipschitz condition (4.1) to get the following estimate for $\varepsilon_{1} \leq 1$ :

$$
\begin{aligned}
\left\|g\left(\nabla V^{n+1}, \mathbf{T}^{n}\right)\right\|_{C_{b}\left([0, T) ; H^{s+1}\right)} & \leq L\left(\left\|V^{n+1}\right\|_{C_{b}\left([0, \mathrm{~T}) ; H^{s+2}\right)}^{2}+\left\|\mathbf{T}^{n}\right\|_{C_{b}\left([0, T) ; H^{s+1}\right)}^{2}\right) \\
& \leq L\left(\left\|V^{n+1}\right\|_{C_{b}\left([0, \mathrm{~T}) ; H^{s+2}\right)}+\left\|\mathbf{T}^{n}\right\|_{C_{b}\left([0, \mathrm{~T}) ; H^{s+1}\right)}^{2}\right) .
\end{aligned}
$$

We also have

$$
\left\|\nabla V^{n+1}+\left(\nabla V^{n+1}\right)^{\mathrm{T}}\right\|_{C_{b}\left([0, \mathrm{~T}) ; H^{s+1}\right)} \leq 2\left\|V^{n+1}\right\|_{C_{b}\left([0, \mathrm{~T}) ; H^{s+2}\right)}
$$

Now combining the above inequalities we obtain the estimate (4.27).

In the next lemma we show that if the initial value $\mathbf{T}_{0}$ and the body force are sufficiently small, then the sequence $\left(V^{n}, q^{n}, p^{n}, \mathbf{T}^{n}\right)$ is bounded independently of $n$.

Lemma 4.6 Let $T \in(0, \infty]$. Assume that there exists $\varepsilon>0$ sufficiently small such that

$$
\left\|\mathbf{T}_{0}\right\|_{H^{s+1}}+\|\mathbf{f}\|_{C_{b}\left([0, T) ; H^{s+1}\right)}+\left\|\frac{\partial}{\partial t} \mathbf{f}\right\|_{C_{b}\left([0, T) ; H^{s}\right)} \leq \varepsilon,
$$

and so $\left\|p_{0}\right\|_{H^{s+1}}$ is also $\leq \varepsilon$. Then, there is $C>0$ independent of $n$ such that the sequence $\left(V^{n}, q^{n}, p^{n}, \mathbf{T}^{n}\right)$ is bounded by $C \varepsilon$ in the space

$$
C_{b}\left([0, T) ; H^{s+2}(\Omega)\right) \times C_{b}\left([0, T) ; H^{s+1}(\Omega)\right) \times C_{b}\left([0, T) ; H^{s+1}(\Omega)\right) \times C_{b}\left([0, T) ; H^{s+1}(\Omega)\right) .
$$


Proof. Let $\alpha_{n}, \beta_{n}, \gamma_{n}$ and $\lambda_{n}$ be bounds at iteration $n$ for $\left\|V^{n}\right\|_{C_{b}\left([0, \mathrm{~T}) ; H^{s+2}\right)},\left\|q^{n}\right\|_{C_{b}\left([0, \mathrm{~T}) ; H^{s+1}\right)}$, $\left\|p^{n}\right\|_{C_{b}\left([0, \mathrm{~T}) ; H^{s+1}\right)}$ and $\left\|\mathbf{T}^{n}\right\|_{C_{b}\left([0, \mathrm{~T}) ; H^{s+1}\right)}$, respectively. Let $\sigma_{n}=\alpha_{n}+\gamma_{n}+\lambda_{n}$.

From the estimates (4.19), (4.26) and (4.27), we find that

$$
\begin{aligned}
& \alpha_{n+1}+\beta_{n+1} \leq C_{1}\left(\alpha_{n}^{2}+\gamma_{n}^{2}+\lambda_{n}^{2}+\varepsilon\right), \\
& \gamma_{n+1} \leq C_{2}\left(\varepsilon+\beta_{n+1}\right), \\
& \lambda_{n+1} \leq C_{3}\left(\varepsilon+\alpha_{n+1}+\lambda_{n}^{2}\right),
\end{aligned}
$$

where $C_{1}, C_{2}$ and $C_{3}$ are non-negative constants and all independent of $n$.

Multiplying the inequality (4.31) by $1+C_{3}$ and (4.32) by $\frac{1+C_{3}}{C_{2}}$, and then adding them together to (4.33), leads to

$$
\sigma_{n+1} \leq a \varepsilon+b \sigma_{n}^{2}
$$

where

$$
a=\frac{1+2 C_{3}+C_{1}+C_{1} C_{3}}{\min \left\{1, \frac{1+C_{3}}{C_{2}}\right\}}, \quad b=\frac{C_{1}\left(1+C_{3}\right)+C_{3}}{\min \left\{1, \frac{1+C_{3}}{C_{2}}\right\}} .
$$

Since $\sigma_{0} \leq(1+|\lambda|) \varepsilon$, then if $C>\max \{1+|\lambda|, a\}$, we have $\sigma_{0} \leq C \varepsilon$ and for

$$
\varepsilon \leq \frac{C-a}{b C^{2}}
$$

we can show by the principle of induction that

$$
\sigma_{n} \leq C \varepsilon \text { for all } n \in \mathbb{N} \text {. }
$$

Therefore, from the inequalities (4.32) and (4.36), there exists $C>0$ independent of $n$ such that

$$
\left\|V^{n}\right\|_{C_{b}\left([0, \mathrm{~T}) ; H^{s+2}\right)}+\left\|q^{n}\right\|_{C_{b}\left([0, \mathrm{~T}) ; H^{s+1}\right)}+\left\|p^{n}\right\|_{C_{b}\left([0, \mathrm{~T}) ; H^{s+1}\right)}+\left\|\mathbf{T}^{n}\right\|_{C_{b}\left([0, \mathrm{~T}) ; H^{s+1}\right)} \leq C \varepsilon
$$

\subsubsection{Convergence}

Let us consider the sequence $\left(\widetilde{V}^{n}, \widetilde{q}^{n}, \widetilde{p}^{n}, \widetilde{\mathbf{T}}^{n}\right)$ formed by the difference between two successive iterations

$$
\left(\widetilde{V}^{n}, \widetilde{q}^{n}, \widetilde{p}^{n}, \widetilde{\mathbf{T}}^{n}\right)=\left(V^{n}-V^{n-1}, q^{n}-q^{n-1}, p^{n}-p^{n-1}, \mathbf{T}^{n}-\mathbf{T}^{n-1}\right)
$$


We subtract the equations obtained at iteration $n$ in problems (4.16), (4.17) and (4.18) from the equations obtained at iteration $n+1$. We note the following identity:

$$
\left(V^{n+1} \cdot \nabla\right) p^{n+1}-\left(V^{n} \cdot \nabla\right) p^{n}=\left(V^{n+1} \cdot \nabla\right) \widetilde{p}^{n+1}+\left(\widetilde{V}^{n+1} \cdot \nabla\right) p^{n}
$$

and similar identities for such terms as

$$
\begin{aligned}
& \left(V^{n+1} \cdot \nabla\right) \mathbf{T}^{n+1}-\left(V^{n} \cdot \nabla\right) \mathbf{T}^{n}, \\
& \left(\frac{\partial V^{n+1}}{\partial x_{j}} \cdot \nabla\right) \mathbf{T}_{i j}^{n+1}-\left(\frac{\partial V^{n}}{\partial x_{j}} \cdot \nabla\right) \mathbf{T}_{i j}^{n}, \\
& \left(\nabla V^{n+1}\right)^{\mathrm{T}} \nabla p^{n+1}-\left(\nabla V^{n}\right)^{\mathrm{T}} \nabla p^{n} .
\end{aligned}
$$

The new sequence $\left(\widetilde{V}^{n+1}, \widetilde{q}^{n+1}, \widetilde{p}^{n+1}, \widetilde{\mathbf{T}}^{n+1}\right)$ satisfies the problems (4.16), (4.17) and (4.18) with different right hand side in their first equations and different initial conditions. The problem satisfied by $\left(\widetilde{V}^{n+1}, \widetilde{q}^{n+1}\right)$ is

$$
\begin{array}{ll}
-\mu \Delta \tilde{V}^{n+1}+\nabla \tilde{q}^{n+1}= & -\sum_{j=1}^{3}\left[\left(\frac{\partial V^{n}}{\partial x_{j}} \cdot \nabla\right) \widetilde{\mathbf{T}}_{i j}^{n}+\left(\frac{\partial \widetilde{V}^{n}}{\partial x_{j}} \cdot \nabla\right) \mathbf{T}_{i j}^{n-1}\right] \\
& +\left(\nabla V^{n}\right)^{\mathrm{T}} \cdot \nabla \widetilde{p}^{n}+\left(\nabla \widetilde{V}^{n}\right)^{\mathrm{T}} \cdot \nabla p^{n-1}-\left(\tilde{V}^{n} \cdot \nabla\right) \mathbf{f} \\
& +\operatorname{div}\left(g\left(\nabla V^{n}, \mathbf{T}^{n}\right)\right)-\operatorname{div}\left(g\left(\nabla V^{n-1}, \mathbf{T}^{n-1}\right)\right), \\
\operatorname{div} \tilde{V}^{n+1}=0 & \text { in } \Omega \times[0, \mathrm{~T}), \\
\tilde{V}^{n+1}=0 & \text { on } \partial \Omega \times[0, \mathrm{~T}) .
\end{array}
$$

In the same way, we obtain the problem satisfied by $\widetilde{p}^{n+1}$

$$
\begin{aligned}
& \frac{\partial}{\partial t} \tilde{p}^{n+1}+\left(V^{n+1} \cdot \nabla\right) \widetilde{p}^{n+1}+\lambda \tilde{p}^{n+1}=\tilde{q}^{n+1}-\left(\widetilde{V}^{n+1} \cdot \nabla\right) p^{n}, \\
& \tilde{p}^{n+1}(\cdot, 0)=0 \quad \text { in } \Omega,
\end{aligned}
$$

and the problem satisfied by $\widetilde{\mathbf{T}}^{n+1}$

$$
\begin{aligned}
& \frac{\partial \tilde{\mathbf{T}}^{n+1}}{\partial t}+\left(V^{n+1} \cdot \nabla\right) \widetilde{\mathbf{T}}^{n+1}+\lambda \tilde{\mathbf{T}}^{n+1}=-\left(\widetilde{V}^{n+1} \cdot \nabla\right) \mathbf{T}^{n}+ \\
& \quad \mu\left(\nabla \tilde{V}^{n+1}+\left(\nabla \tilde{V}^{n+1}\right)^{\mathrm{T}}\right)+g\left(\nabla V^{n+1}, \mathbf{T}^{n}\right)-g\left(\nabla V^{n}, \mathbf{T}^{n-1}\right), \\
& \tilde{\mathbf{T}}^{n+1}(\cdot, 0)=0 \quad \text { in } \Omega .
\end{aligned}
$$


Using the estimates obtained in the Lemmas 4.3, 4.4 and 4.5, and the result in Lemma 4.6, we obtain the estimates

$$
\begin{aligned}
& \left\|\tilde{V}^{n+1}\right\|_{C_{b}\left([0, \mathrm{~T}) ; H^{s+1}\right)}+\left\|\tilde{q}^{n+1}\right\|_{C_{b}\left([0, \mathrm{~T}) ; H^{s}\right)} \leq \\
& K_{1} \varepsilon\left(\left\|\tilde{V}^{n}\right\|_{C_{b}\left([0, \mathrm{~T}) ; H^{s+1}\right)}+\left\|\tilde{p}^{n}\right\|_{C_{b}\left([0, \mathrm{~T}) ; H^{s}\right)}+\left\|\tilde{\mathbf{T}}^{n}\right\|_{C_{b}\left([0, \mathrm{~T}) ; H^{s}\right)}\right) \\
& \left\|\tilde{p}^{n+1}\right\|_{C_{b}\left([0, \mathrm{~T}) ; H^{s}\right)} \leq K_{2}\left(\left\|\tilde{q}^{n+1}\right\|_{C_{b}\left([0, \mathrm{~T}) ; H^{s}\right)}+\varepsilon\left\|\tilde{V}^{n+1}\right\|_{C_{b}\left([0, \mathrm{~T}) ; H^{s+1}\right)}\right) \\
& \left\|\tilde{\mathbf{T}}^{n+1}\right\|_{C_{b}\left([0, \mathrm{~T}) ; H^{s}\right)} \leq K_{3}\left(\left\|\tilde{V}^{n+1}\right\|_{C_{b}\left([0, \mathrm{~T}) ; H^{s+1}\right)}+\varepsilon\left\|\tilde{\mathbf{T}}^{n}\right\|_{C_{b}\left([0, \mathrm{~T}) ; H^{s}\right)}\right)
\end{aligned}
$$

where $K_{1}, K_{2}$ and $K_{3}$ are non-negative constants independent of $n$, and $\varepsilon>0$ is sufficiently small.

Combining the inequalities (4.43), (4.44) and (4.45), leads to the following estimate for some $K>0$ :

$$
\begin{aligned}
& \left\|\tilde{V}^{n+1}\right\|_{C_{b}\left([0, T) ; H^{s+1}\right)}+\left\|\tilde{p}^{n+1}\right\|_{C_{b}\left([0, T) ; H^{s}\right)}+\left\|\tilde{\mathbf{T}}^{n+1}\right\|_{C_{b}\left([0, T) ; H^{s}\right)} \leq \\
& K \varepsilon\left(\left\|\tilde{V}^{n}\right\|_{C_{b}\left([0, T) ; H^{s+1}\right)}+\left\|\tilde{p}^{n}\right\|_{C_{b}\left([0, T) ; H^{s}\right)}+\left\|\tilde{\mathbf{T}}^{n}\right\|_{C_{b}\left([0, T) ; H^{s}\right)}\right) .
\end{aligned}
$$

If we choose $\varepsilon$ so that $K \varepsilon<1$, we deduce from the previous inequality and estimate (4.43) that the sequence $\left(V^{n}, q^{n}, \mathbf{T}^{n}, p^{n}\right)$ is a Cauchy sequence in a bounded subset of the space

$$
L^{\infty}\left(0, \mathrm{~T} ; H^{s+2}(\Omega)\right) \times L^{\infty}\left(0, \mathrm{~T} ; H^{s+1}(\Omega)\right) \times L^{\infty}\left(0, \mathrm{~T} ; H^{s+1}(\Omega)\right) \times L^{\infty}\left(0, \mathrm{~T} ; H^{s+1}(\Omega)\right)
$$

equipped with the norm of

$$
C_{b}\left(0, \mathrm{~T} ; H^{s+1}(\Omega)\right) \times C_{b}\left(0, \mathrm{~T} ; H^{s}(\Omega)\right) \times C_{b}\left(0, \mathrm{~T} ; H^{s}(\Omega)\right) \times C_{b}\left(0, \mathrm{~T} ; H^{s}(\Omega)\right),
$$

and hence converges to a limit $(V, q, \mathbf{T}, p)$, satisfying the equations (4.8)-(4.13). Moreover, by the estimate (4.37), this limit has norm bounded by a constant times $\varepsilon$. Although the contraction argument guarantees only that the limit is in the space given by (4.47), continuity in time follows in a standard fashion from the fact that the limit function satisfies the differential equation.

The results obtained above can be summarized in the following theorem. 
Theorem 4.7 Let $T \in(0, \infty]$ and let $\varepsilon>0$ be sufficiently small.

If

$$
\left\|\mathbf{T}_{0}\right\|_{H^{s+1}}+\|\mathbf{f}\|_{C_{b}\left([0, T), H^{s+1}\right)}+\left\|\frac{\partial}{\partial t} \mathbf{f}\right\|_{\left.C_{b}\left([0, T), H^{s}\right)\right)} \leq \varepsilon,
$$

with the condition that $\operatorname{div}\left(\mathbf{T}_{0}\right)-\mathbf{f}(\cdot, 0)$ is a gradient, then there exists a unique solution $(V, q, \mathbf{T}, p)$ of the problem (4.8)-(4.13) in the space

$$
\begin{aligned}
& C_{b}\left([0, T) ; H^{s+2}(\Omega)\right) \times C_{b}\left([0, T) ; H^{s+1}(\Omega) \cap L_{0}^{2}(\Omega)\right) \\
& \times C_{b}\left([0, T) ; H^{s+1}(\Omega)\right) \times C_{b}\left([0, T) ; H^{s+1}(\Omega) \cap L_{0}^{2}(\Omega)\right) .
\end{aligned}
$$

Corollary 4.8 Under same conditions in Theorem 4.7, there exists a unique solution $(V, p, \mathbf{T})$ for the problem (1.12)-(1.16) in the following space:

$$
C_{b}\left([0, T) ; H^{s+2}(\Omega)\right) \times C_{b}\left([0, T) ; H^{s+1}(\Omega) \cap L_{0}^{2}(\Omega)\right) \times C_{b}\left([0, T) ; H^{s+1}(\Omega)\right) .
$$




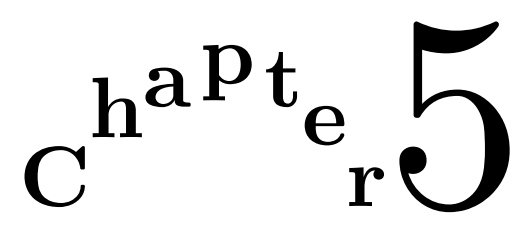

\section{Unsteady simple shear flow with arbitrary initial data}

\section{Contents}

5.1 Problem formulation $\ldots \ldots \ldots \ldots$

5.2 Reduction to iterative subproblems ........... 55

5.3 Existence and uniqueness . . . . . . . . . . . . 56

5.4 Convergence of the scheme . . . . . . . . . . . 61

We propose to study in this chapter the existence and uniqueness of solutions for an unsteady simple shear flow problem in the case where the initial data are arbitrary.

\subsection{Problem formulation}

We consider a simple shear flow problem in the unsteady type, where the velocity of the fluid is only in the $x$-direction and changing only in the $y$-direction, see Section 1.3.3.

$$
V=(u(y, t), 0,0) .
$$


The velocity gradient has only one nonzero component, the shear rate

$$
\kappa(y, t)=\frac{\partial u}{\partial y}(y, t) .
$$

The extra stress tensor $\mathbf{T}$ has four independent components, and is written as follows:

$$
\mathbf{T}=\left(\begin{array}{ccc}
T_{11}(y, t) & T_{12}(y, t) & 0 \\
T_{12}(y, t) & T_{22}(y, t) & 0 \\
0 & 0 & T_{33}(y, t)
\end{array}\right)
$$

Hence the divergence of $\mathbf{T}$ is

$$
\operatorname{div} \mathbf{T}=\left(\frac{\partial T_{12}}{\partial y}(y, t), \frac{\partial T_{22}}{\partial y}(y, t), 0\right)^{\mathrm{T}}
$$

The equation of the balance of momentum becomes $\operatorname{div} \mathbf{T}-\nabla p=0$, is reduced to

$$
\begin{aligned}
& T_{12}(y, t)=a_{12}(t) y+b_{12}(t), \\
& p \equiv p(x, y, t)=a_{12}(t) x+T_{22}(y, t)+c(t) .
\end{aligned}
$$

We consider an unsteady simple shear flow problem, where the fluid is placed between two parallel plates separated by a distance $h$, and the velocity on each plate is prescribed to be zero. The flow is driven by a pressure gradient, and the total flow rate $F$ is prescribed. The problem can be stated as follows: Find a velocity $u(y, t)$ and extra stress tensor $\mathbf{T}(y, t)$ of the form given in (5.3), satisfying the equations

$$
\begin{array}{lc}
-\mu \frac{\partial u}{\partial y}(y, t)+\frac{\partial}{\partial t} T_{12}(y, t)+\lambda T_{12}(y, t)=g_{12} & \left(\frac{\partial u}{\partial y}(y, t), T_{\mathbf{n}}(y, t), T_{12}(y, t)\right) \\
& \text { in }(0, h) \times[0, \mathrm{~T}], \mathrm{T} \in(0, \infty), \\
\begin{array}{ll}
u(0, t)=u(h, t)=0, \int_{0}^{h} u(y, t) d y=F & \text { in }[0, \mathrm{~T}], \\
T_{12}(y, 0)=a_{12}^{0} y+b_{12}^{0}, \quad \text { in }(0, h), & \\
\frac{\partial T_{\mathbf{n}}}{\partial t}(y, t)+\lambda T_{\mathbf{n}}(y, t)=g_{\mathbf{n}}\left(\frac{\partial u}{\partial y}(y, t), T_{\mathbf{n}}(y, t), T_{12}(y, t)\right) \\
T_{\mathbf{n}}(y, 0)=T_{\mathbf{n}}^{0}(y) \quad \text { in }(0, h),
\end{array}
\end{array}
$$


where $T_{\mathbf{n}}(y, t)$ denotes the diagonal components of the extra stress tensor $\mathbf{T}$, which are called normal extra stresses

$$
T_{\mathbf{n}}(y, t)=\left(T_{11}(y, t), T_{22}(y, t), T_{33}(y, t)\right)
$$

and

$$
g_{\mathbf{n}}=\left(g_{11}, g_{22}, g_{33}\right) \text {. }
$$

The functions $g_{11}, g_{22}, g_{33}, g_{12}:(\kappa, N) \in \mathbb{R} \times \mathbb{R}^{3+1} \rightarrow \mathbb{R}$ are assumed to be of class $C^{s+3}$ on $\mathbb{R} \times \mathbb{R}^{3+1}, s$ is an integer $\geq 0$. In this case they map $H^{s+1}(0, h) \times\left(H^{s+1}(0, h)\right)^{3+1}$ into $H^{s+1}(0, h)$.

Introducing the new functions

$$
\begin{aligned}
& q(t)=\frac{d}{d t} a_{12}(t)+\lambda a_{12}(t), \\
& w(t)=\frac{d}{d t} b_{12}(t)+\lambda b_{12}(t),
\end{aligned}
$$

the equations (5.6) and (5.7) can be rewritten as

$$
\begin{aligned}
& -\mu \kappa(y, t)+q(t) y+w(t)=g_{12}\left(\kappa(y, t), T_{\mathbf{n}}(y, t), T_{12}(y, t)\right) \quad \text { in }(0, h) \times[0, \mathrm{~T}], \\
& \int_{0}^{h} \kappa(y, t) d y=0, \int_{0}^{h} y \kappa(y, t) d y=-F \quad \text { in }[0, \mathrm{~T}] .
\end{aligned}
$$

The problem of finding a solution $(\kappa(y, 0), q(0), w(0))$ of the equations (5.15)-(5.16) at the initial time $t=0$ may be ill-posed for some models. However, for most popular models, such a solution exists for any given initial extra stress tensor $\mathbf{T}^{0}(y)$.

We shall formulate a condition that guarantees the existence and uniqueness of solutions to problem (5.15)-(5.16) at initial time $t=0$. For each $y \in[0, h]$, we assume the strict monotonicity (increasing) of the functions

$$
\begin{aligned}
\mathbb{R} & \rightarrow \mathbb{R} \\
\kappa & \mapsto \mu \kappa+g_{12}\left(\kappa, T_{\mathbf{n}}^{0}(y), T_{12}^{0}(y)\right) .
\end{aligned}
$$

From this and the assumption that $g_{12}$ is of class $C^{s+3}$ on $\mathbb{R} \times \mathbb{R}^{3+1}$, we have

$$
\mu+\frac{\partial g_{12}}{\partial \kappa}\left(\kappa, T_{\mathbf{n}}^{0}(y), T_{12}^{0}(y)\right)>0, \text { for all } y \in[0, h] \text { and } \kappa \in \mathbb{R} .
$$

In addition, we assume that

$$
\lim _{|\kappa| \rightarrow \infty}\left|\mu \kappa+g_{12}\left(\kappa, T_{\mathbf{n}}^{0}(y), T_{12}^{0}(y)\right)\right|=\infty \text { for all } y \in[0, h]
$$


Lemma 5.1 Let $s \geq 0$ and $T_{\mathbf{n}}^{0}, T_{12}^{0} \in H^{s}([0, h])$. Under the assumptions of strict monotonicity and the condition (5.19), the problem (5.15)-(5.16) at $t=0$ has a unique solution

$$
(\kappa(y, 0), q(0), w(0)) \in H^{s+1}([0, h]) \times \mathbb{R} \times \mathbb{R} .
$$

For simplicity in writing some of the formulas, the following notations will be used

$$
\kappa^{0}(y):=\kappa(y, 0), q^{0}:=q(0) \text { and } w^{0}:=w(0) .
$$

Proof. Since the function $g_{12}$ satisfies the conditions (5.18) and (5.19), then the global inverse function theorem (see Section 2.3, Theorem 2.13) can be applied to rewrite $\kappa^{0}(y)$ as a function of $q^{0}, w^{0}$ and $y$ in the form

$$
\kappa^{0}(y)=\phi\left(q^{0} y+w^{0}, T_{\mathbf{n}}^{0}(y), T_{12}^{0}(y)\right), y \in[0, h]
$$

where $\phi$ denotes the inverse function of $\kappa \mapsto \varkappa=\mu \kappa+g_{12}\left(\kappa, T_{\mathbf{n}}^{0}(y), T_{12}^{0}(y)\right)$. The function $\phi$ inherits the assumptions (5.18) and (5.19), so we have

$$
\begin{gathered}
\frac{\partial \phi}{\partial \varkappa}\left(\varkappa, T_{\mathbf{n}}^{0}(y), T_{12}^{0}(y)\right)=\frac{1}{\mu+\frac{\partial g_{12}}{\partial \kappa}\left(\kappa, T_{\mathbf{n}}^{0}(y), T_{12}^{0}(y)\right)}>0 \\
\text { for all } y \in[0, h] \text { and } \varkappa \in \mathbb{R}, \\
\lim _{|\varkappa| \rightarrow \infty}\left|\phi\left(\varkappa, T_{\mathbf{n}}^{0}(y), T_{12}^{0}(y)\right)\right|=\infty \text { for all } y \in[0, h] .
\end{gathered}
$$

We note that, since $\phi$ is increasing, $\phi$ will have the same sign as $\varkappa$ if $|\varkappa|$ is large. Now, applying the conditions (5.16) at $t=0$, we obtain

$$
\begin{gathered}
f_{1}\left(q^{0}, w^{0}\right):=\int_{0}^{h} \phi\left(q^{0} y+w^{0}, T_{\mathbf{n}}^{0}(y), T_{12}^{0}(y)\right) d y=0, \\
f_{2}\left(q^{0}, w^{0}\right):=\int_{0}^{h} y \phi\left(q^{0} y+w^{0}, T_{\mathbf{n}}^{0}(y), T_{12}^{0}(y)\right) d y=-F .
\end{gathered}
$$

The Jacobian matrix corresponding to this system is

$$
J_{\left(f_{1}, f_{2}\right)}\left(q^{0}, w^{0}\right)=\left[\begin{array}{cc}
J_{11}\left(q^{0}, w^{0}\right) & J_{12}\left(q^{0}, w^{0}\right) \\
J_{21}\left(q^{0}, w^{0}\right) & J_{22}\left(q^{0}, w^{0}\right)
\end{array}\right],
$$

where

$$
\begin{aligned}
& J_{11}\left(q^{0}, w^{0}\right)=J_{22}\left(q^{0}, w^{0}\right):=\int_{0}^{h} y \xi^{0}(y) d y \\
& J_{12}\left(q^{0}, w^{0}\right):=\int_{0}^{h} \xi^{0}(y) d y, J_{21}\left(q^{0}, w^{0}\right):=\int_{0}^{h} y^{2} \xi^{0}(y) d y,
\end{aligned}
$$


with

$$
\xi^{0}(y):=\frac{\partial \phi}{\partial \varkappa}\left(q^{0} y+w^{0}, T_{\mathbf{n}}^{0}(y), T_{12}^{0}(y)\right) .
$$

Therefore, the Jacobian determinant is

$$
\operatorname{det}\left(J_{\left(f_{1}, f_{2}\right)}\left(q^{0}, w^{0}\right)\right)=\left[J_{11}\left(q^{0}, w^{0}\right)\right]^{2}-J_{21}\left(q^{0}, w^{0}\right) J_{12}\left(q^{0}, w^{0}\right) .
$$

Note that the following inequalities hold for all $q^{0}, w^{0} \in \mathbb{R}$ :

$$
\frac{J_{11}\left(q^{0}, w^{0}\right)}{J_{12}\left(q^{0}, w^{0}\right)} \leq h, \quad \frac{J_{21}\left(q^{0}, w^{0}\right)}{J_{12}\left(q^{0}, w^{0}\right)} \leq h^{2}, \quad \frac{J_{21}\left(q^{0}, w^{0}\right)}{J_{11}\left(q^{0}, w^{0}\right)} \leq h .
$$

The Jacobian determinant can be rewritten in the following way:

$$
\operatorname{det}\left(J_{\left(f_{1}, f_{2}\right)}\left(q^{0}, w^{0}\right)\right)=-\int_{0}^{h}\left(y \sqrt{J_{12}\left(q^{0}, w^{0}\right)}-\frac{J_{11}\left(q^{0}, w^{0}\right)}{\sqrt{J_{12}\left(q^{0}, w^{0}\right)}}\right)^{2} \xi^{0}(y) d y,
$$

which shows that it is non-positive for all $q^{0}, w^{0} \in \mathbb{R}$ since $\xi^{0}(y)>0$.

Next, we show that

$$
\lim _{\left\|\left(q^{0}, w^{0}\right)\right\| \rightarrow \infty}\left\|\left(f_{1}\left(q^{0}, w^{0}\right), f_{2}\left(q^{0}, w^{0}\right)\right)\right\|=\infty .
$$

Multiplying $f_{1}\left(q^{0}, w^{0}\right)$ by $w^{0}$ and $f_{2}\left(q^{0}, w^{0}\right)$ by $q^{0}$ we get

$$
w^{0} f_{1}\left(q^{0}, w^{0}\right)+q^{0} f_{2}\left(q^{0}, w^{0}\right):=\int_{0}^{h}\left(q^{0} y+w^{0}\right) \phi\left(q^{0} y+w^{0}, T_{\mathbf{n}}^{0}(y), T_{12}^{0}(y)\right) d y .
$$

By Cauchy-Schwarz inequality we have

$$
\sqrt{\left[f_{1}\left(q^{0}, w^{0}\right)\right]^{2}+\left[f_{2}\left(q^{0}, w^{0}\right)\right]^{2}} \geq \frac{\left|w^{0} f_{1}\left(q^{0}, w^{0}\right)+q^{0} f_{2}\left(q^{0}, w^{0}\right)\right|}{\sqrt{\left(q^{0}\right)^{2}+\left(w^{0}\right)^{2}}} .
$$

Using polar coordinates, $q^{0}=r \cos \theta, w^{0}=r \sin \theta$, we see that

$$
\frac{w^{0} f_{1}+q^{0} f_{2}}{\sqrt{\left(q^{0}\right)^{2}+\left(w^{0}\right)^{2}}}=\int_{0}^{h}(y \cos \theta+\sin \theta) \phi\left(r(y \cos \theta+\sin \theta), T_{\mathbf{n}}^{0}(y), T_{12}^{0}(y)\right) d y
$$

For $\tan \theta \notin[-h, 0]$ we have $|y \cos \theta+\sin \theta|>0$ for all $y \in[0, h]$. Hence, if $r$ is large enough, then

$$
(y \cos \theta+\sin \theta) \phi\left(r(y \cos \theta+\sin \theta), T_{\mathbf{n}}^{0}(y), T_{12}^{0}(y)\right) \geq 0 \text { for all } y \in[0, h] \text {. }
$$


If $\tan \theta \in[-h, 0]$, then for $\varepsilon>0$ there exists $r_{\varepsilon}$ such that for $r \geq r_{\varepsilon}$ we have

$$
\int_{-\tan \theta-\varepsilon}^{-\tan \theta+\varepsilon}(y \cos \theta+\sin \theta) \phi\left(r(y \cos \theta+\sin \theta), T_{\mathbf{n}}^{0}(y), T_{12}^{0}(y)\right) d y \geq 0,
$$

and

$$
\begin{aligned}
& (y \cos \theta+\sin \theta) \phi\left(r(y \cos \theta+\sin \theta), T_{\mathbf{n}}^{0}(y), T_{12}^{0}(y)\right) \geq 0 \\
& \quad \text { for all } y \in[0, h] \backslash[-\tan \theta-\varepsilon,-\tan \theta+\varepsilon] .
\end{aligned}
$$

It follows that for $\varepsilon>0$, there exists an interval $I_{\varepsilon} \subset[0, h]$ and $r_{\varepsilon}>0$ such that if $r \geq r_{\varepsilon}$, then

$$
\begin{aligned}
& \frac{w^{0} f_{1}+q^{0} f_{2}}{\sqrt{\left(q^{0}\right)^{2}+\left(w^{0}\right)^{2}}} \geq \int_{I_{\varepsilon}}\left|(y \cos \theta+\sin \theta) \phi\left(r(y \cos \theta+\sin \theta), T_{\mathbf{n}}^{0}(y), T_{12}^{0}(y)\right)\right| d y, \\
& \text { with }|y \cos \theta+\sin \theta| \geq \varepsilon \text { for all } y \in I_{\varepsilon} .
\end{aligned}
$$

This shows that

$$
\lim _{\left\|\left(q^{0}, w^{0}\right)\right\| \rightarrow \infty} \frac{\left|w^{0} f_{1}\left(q^{0}, w^{0}\right)+q^{0} f_{2}\left(q^{0}, w^{0}\right)\right|}{\sqrt{\left(q^{0}\right)^{2}+\left(w^{0}\right)^{2}}}=\infty .
$$

Thus, from the inequality (5.26), the limit in (5.25) holds. Consequently, using the global inverse function theorem (Theorem 2.13), the constants $q^{0}$ and $w^{0}$ can be determined uniquely. This finishes the proof of the lemma.

\subsection{Reduction to iterative subproblems}

To prove the existence result for the problem (5.6)-(5.10), we adopt the method we used in the three-dimensional case. We reduce our problem to a sequence of subproblems whose solutions form a sequence converging to a solution for the problem (5.6)-(5.10). The sequence of the subproblems will be constructed in such a way that the solutions of earlier problems will be used to solve the later ones.

We initialize the scheme starting with

$$
\kappa^{0}(y, t)=\kappa(y, 0), T_{12}^{0}(y, 0)=a_{12}^{0} y+b_{12}^{0} \text { and } T_{\mathbf{n}}^{0}(y, t)=T_{\mathbf{n}}(y, 0) .
$$


Then, keeping $y$ fixed in the interval $[0, h]$, we solve the following problem for $\kappa^{n+1}(y, t)$, $q^{n+1}(t)$ and $w^{n+1}(t)$ :

$$
\begin{aligned}
\mu \kappa^{n+1}(y, t)+g_{12} & \left(\kappa^{n+1}(y, t), T_{\mathbf{n}}^{n}(y, t), T_{12}^{n}(y, t)\right) \\
& =q^{n+1}(t) y+w^{n+1}(t) \text { in }(0, h) \times[0, \mathrm{~T}], \\
\int_{0}^{h} \kappa^{n+1}(y, t) d y= & 0, \int_{0}^{h} y \kappa^{n+1}(y, t) d y=0 \quad \text { in }[0, \mathrm{~T}] .
\end{aligned}
$$

Note that

$$
u^{n+1}(y, t)=\int_{0}^{y} \kappa^{n+1}\left(y_{1}, t\right) d y_{1} .
$$

For now, let us assume that the problem (5.28) has a solution $\left(\kappa^{n+1}, q^{n+1}, w^{n+1}\right)$ in some appropriate space. Next, we solve for $a_{12}^{n+1}$ and $b_{12}^{n+1}$ the following differential equations:

$$
\left.\begin{array}{ll}
\frac{d}{d t} a_{12}^{n+1}(t)+\lambda a_{12}^{n+1}(t)=q^{n+1}(t) & t \in[0, \mathrm{~T}], \\
a_{12}^{n+1}(0)=a_{12}^{0}, & \\
\frac{d}{d t} b_{12}^{n+1}(t)+\lambda b_{12}^{n+1}(t)=w^{n+1}(t) & t \in[0, \mathrm{~T}], \\
b_{12}^{n+1}(0)=b_{12}^{0}, &
\end{array}\right\}
$$

and for $T_{\mathbf{n}}^{n+1}$ the evolution problem:

$$
\left.\begin{array}{lr}
\frac{\partial T_{\mathbf{n}}^{n+1}}{\partial t}(y, t)+\lambda T_{\mathbf{n}}^{n+1}(y, t)=g_{\mathbf{n}}\left(\kappa^{n+1}(y, t), T_{\mathbf{n}}^{n}(y, t), T_{12}^{n}(y, t)\right) \\
T_{\mathbf{n}}^{n+1}(y, 0)=T_{\mathbf{n}}^{0}(y) & \text { in }(0, h) \times[0, \mathrm{~T}],
\end{array}\right\}
$$

\subsection{Existence and uniqueness}

We establish the existence and uniqueness of solutions to the scheme that we have already constructed in the last section.

Lemma 5.2 Let $T \in(0, \infty)$; let $s$ be an integer $\geq 0$. Assume that $T_{\mathbf{n}}^{n}, T_{12}^{n}$ are in the space $C\left([0, T], H^{s+1}(0, h)\right)$, and they are close enough to the initial extra stress tensors $T_{\mathbf{n}}^{0}, T_{12}^{0}$, 
respectively. Then there exists a unique solution $\left(\kappa^{n+1}, q^{n+1}, w^{n+1}\right)$ to the problem (5.28), such that

$$
\left(\kappa^{n+1}, q^{n+1}, w^{n+1}\right) \in C\left([0, T], H^{s+1}(0, h)\right) \times C([0, T]) \times C([0, T]),
$$

and satisfies the estimates

$$
\begin{gathered}
\left\|q^{n+1}-q^{0}\right\|_{C([0, T])}+\left\|w^{n+1}-w^{0}\right\|_{C([0, T])} \leq C_{1}\left\|\mathbf{T}^{n}-\mathbf{T}^{0}\right\|_{C\left([0, T], H^{s+1}\right)}, \\
\left\|\kappa^{n+1}-\kappa^{0}\right\|_{C\left([0, T], H^{s+1}\right)} \leq C_{1}\left\|\mathbf{T}^{n}-\mathbf{T}^{0}\right\|_{C\left([0, T], H^{s+1}\right)},
\end{gathered}
$$

where $C_{1}$ is a non-negative constant depending on the small bound for $\left\|\mathbf{T}^{n}-\mathbf{T}_{0}\right\|$, but otherwise independent of $n$ and $T$.

Proof. Since the function $g_{12}$ is assumed to satisfy the conditions (5.18) and (5.19), by applying the global inverse function theorem (Theorem 2.13), $\kappa^{n+1}$ can be expressed in terms of $q^{n+1} y+w^{n+1}$.

$$
\kappa^{n+1}(y, t)=\phi\left(q^{n+1}(t) y+w^{n+1}(t), T_{\mathbf{n}}^{n}(y, t), T_{12}^{n}(y, t)\right),
$$

where $\phi$ is as defined in Lemma 5.1, denotes the inverse function of $\kappa \mapsto \varkappa=\mu \kappa+$ $g_{12}\left(\kappa, T_{\mathbf{n}}^{n}, T_{12}^{n}\right)$.

The conditions of the problem (5.28), therefore, can be rewritten in the form

$$
\begin{aligned}
f_{1}\left(q^{n+1}, w^{n+1}\right) & :=\int_{0}^{h} \phi\left(q^{n+1}(t) y+w^{n+1}(t), T_{\mathbf{n}}^{n}(y, t), T_{12}^{n}(y, t)\right) d y=0, \\
f_{2}\left(q^{n+1}, w^{n+1}\right) & :=\int_{0}^{h} y \phi\left(q^{n+1}(t) y+w^{n+1}(t), T_{\mathbf{n}}^{n}(y, t), T_{12}^{n}(y, t)\right) d y=0 .
\end{aligned}
$$

By the proof of Lemma 5.1, the system (5.36)-(5.37) at the initial time $t=0$ has a unique solution $\left(q^{0}, w^{0}\right)$, and the corresponding Jacobian determinant does not vanish at any point. It follows from the implicit function theorem that this system has a unique solution in a small neighborhood of $\left(T_{12}^{0}(y), T_{\mathbf{n}}^{0}(y)\right)$, expressed in terms of $T_{\mathbf{n}}^{n}(y, t)$ and $T_{12}^{n}(y, t)$,

$$
q^{n+1}=\phi_{1}\left(T_{\mathbf{n}}^{n}(y, t), T_{12}^{n}(y, t)\right), w^{n+1}=\phi_{2}\left(T_{\mathbf{n}}^{n}(y, t), T_{12}^{n}(y, t)\right) .
$$

Hence, for $\left(T_{12}^{n}, T_{\mathbf{n}}^{n}\right)$ close enough to $\left(T_{12}^{0}, T_{\mathbf{n}}^{0}\right)$, the problem (5.28) has a unique solution $\left(\kappa^{n+1}, q^{n+1}, w^{n+1}\right)$. Next, we show that this solution satisfies the estimates (5.33) and (5.34). Using the derivative formula (2.27) we get

$$
D_{\mathbf{T}^{n}}\left(\phi_{1}, \phi_{2}\right)=-\left(J_{\left(f_{1}, f_{2}\right)}\left(q^{n+1}, w^{n+1}\right)\right)^{-1} \circ D_{\mathbf{T}^{n}}\left(f_{1}, f_{2}\right),
$$


where

$$
\begin{array}{r}
D_{\mathbf{T}^{n}} f_{1}\left(\mathbf{T}^{n}\right)(S)=-\int_{0}^{h} \xi^{n+1}(y, t) \frac{\partial g_{12}}{\partial \mathbf{T}^{n}}\left(\kappa^{n+1}(y, t), \mathbf{T}^{n}(y, t)\right) S(y) d y, \\
D_{\mathbf{T}^{n}} f_{2}\left(\mathbf{T}^{n}\right)(S)=-\int_{0}^{h} y \xi^{n+1}(y, t) \frac{\partial g_{12}}{\partial \mathbf{T}^{n}}\left(\kappa^{n+1}(y, t), \mathbf{T}^{n}(y, t)\right) S(y) d y, \\
S \in L^{2}(0, h), \\
J_{\left(f_{1}, f_{2}\right)}\left(q^{n+1}, w^{n+1}\right)=\left[\begin{array}{ll}
\int_{0}^{h} y \xi^{n+1}(y, t) d y & \int_{0}^{h} \xi^{n+1}(y, t) d y \\
\int_{0}^{h} y^{2} \xi^{n+1}(y, t) d y & \int_{0}^{h} y \xi^{n+1}(y, t) d y
\end{array}\right],
\end{array}
$$

with

$$
\xi^{n+1}(y, t)=1 /\left(\mu+\frac{\partial g_{12}}{\partial \kappa}\left(\kappa^{n+1}(y, t), T_{\mathbf{n}}^{n}(y, t), T_{12}^{n}(y, t)\right)\right) .
$$

Since $g_{12}$ satisfies the condition (5.18), then there exists a constant $a_{1}$ such that

$$
0<\xi^{n+1}(y, t) \leq a_{1}
$$

and proceeding as in the proof of Lemma 5.1, we see that

$$
0<-1 / \operatorname{det}\left(J_{\left(f_{1}, f_{2}\right)}\left(q^{n+1}, w^{n+1}\right)\right) \leq a_{2},
$$

where $a_{2}$ is a constant $>0$ and

$$
\begin{aligned}
\operatorname{det}( & \left.J_{\left(f_{1}, f_{2}\right)}\left(q^{n+1}, w^{n+1}\right)\right)= \\
& \left(\int_{0}^{h} y \xi^{n+1}(y, t) d y\right)^{2}-\left(\int_{0}^{h} y^{2} \xi^{n+1}(y, t) d y\right)\left(\int_{0}^{h} \xi^{n+1}(y, t) d y\right) .
\end{aligned}
$$

Consequently, we see for $\mathbf{T}^{n}$ in the neighborhood of $\mathbf{T}^{0}$ that

$$
\left\|D_{\mathbf{T}^{n}}\left(\phi_{1}, \phi_{2}\right)\left(\mathbf{T}^{n}\right)\right\|_{\mathcal{L}\left(L^{2}(0, h), \mathbb{R}\right)} \leq C, C>0 .
$$

Therefore, by mean value theorem, we get

$$
\left|\phi_{1}\left(\mathbf{T}^{n}\right)-\phi_{1}\left(\mathbf{T}^{0}\right)\right|+\left|\phi_{2}\left(\mathbf{T}^{n}\right)-\phi_{2}\left(\mathbf{T}^{0}\right)\right| \leq C\left\|\mathbf{T}^{n}-\mathbf{T}^{0}\right\|_{L^{2}} .
$$

Hence, it follows that

$$
\left|q^{n+1}-q^{0}\right|+\left|w^{n+1}-w^{0}\right| \leq C\left\|\mathbf{T}^{n}-\mathbf{T}^{0}\right\|_{L^{2}} .
$$

Again, by mean value theorem, we have

$$
\left(\mu+\frac{\partial g_{12}}{\partial \kappa}\left(\kappa_{c}^{n+1}, T_{\mathbf{n}}^{n}, T_{12}^{n}\right)\right)\left(\kappa^{n+1}-\kappa^{0}\right)=\left(q^{n+1}-q^{0}\right) y+\left(w^{n+1}-w^{0}\right)
$$


Combining with (5.39) we obtain

$$
\left\|\kappa^{n+1}-\kappa^{0}\right\|_{H^{s+1}} \leq C_{1}\left\|\mathbf{T}^{n}-\mathbf{T}^{0}\right\|_{H^{s+1}}, \quad C_{1}>0 .
$$

Lemma 5.3 Let $T$ be in $(0, \infty)$ and let $s$ be an integer $\geq 0$. If $q^{n+1}, w^{n+1} \in C([0, T])$, then the problems (5.30) and (5.31) have a unique solution $a_{12}^{n+1} \in C([0, T])$ and $b_{12}^{n+1} \in C([0, T])$, respectively. Moreover, the following estimates hold:

$$
\begin{aligned}
& \left\|a_{12}^{n+1}-a_{12}^{0}\right\|_{C([0, T])} \leq C_{T}\left(\left\|q^{n+1}-q^{0}\right\|_{C([0, T])}+\left|q^{0}-\lambda a_{12}^{0}\right|\right), \\
& \left\|b_{12}^{n+1}-b_{12}^{0}\right\|_{C([0, T])} \leq C_{T}\left(\left\|w^{n+1}-w^{0}\right\|_{C([0, T])}+\left|w^{0}-\lambda b_{12}^{0}\right|\right),
\end{aligned}
$$

where

$$
C_{T}=\frac{1-e^{-\lambda T}}{\lambda}
$$

Proof. The proof follows from the basic results on ordinary differential equations.

Recall that $T_{12}^{n+1}(y, t)=a_{12}^{n+1}(t) y+b_{12}^{n+1}(t)$, see formula (5.4). Hence, multiplying the equation in problem (5.30) by $y$ and adding the equation in problem (5.31), then using the estimates obtained in Lemma 5.3 and combining with (5.33), yields

$$
\left\|T_{12}^{n+1}-T_{12}^{0}\right\|_{C\left([0, \mathrm{~T}], H^{s+1}\right)} \leq C_{\mathrm{T}} C_{1}\left(\left\|\mathbf{T}^{n}-\mathbf{T}^{0}\right\|_{C\left([0, \mathrm{~T}], H^{s+1}\right)}+\varphi_{1}\left(\mathbf{T}^{0}\right)\right)
$$

where $C_{\mathrm{T}}$ is as defined above in (5.42), whereas $C_{1}$ is a non-negative constant independent of $n$ and $\mathrm{T}$, and $\varphi_{1}\left(\mathbf{T}^{0}\right)$ is a positive constant depending on $\mathbf{T}^{0}$. Also, $\varphi_{1}\left(\mathbf{T}^{0}\right)$ is independent of $n$ and $\mathrm{T}$.

Lemma 5.4 Let $T$ be in $(0, \infty), s$ be an integer $\geq 0$ and suppose that $T_{\mathbf{n}}^{0} \in H^{s+1}(0, h)$, $\kappa^{n+1}, T_{\mathbf{n}}^{n}, T_{12}^{n} \in C\left([0, T], H^{s+1}(0, h)\right)$. Then the problem (5.32) has a unique solution $T_{\mathbf{n}}^{n+1} \in$ $C\left([0, T], H^{s+1}(0, h)\right)$. Moreover, there exist constants $K_{1}, K_{2}>0$, such that if

$$
\left\|\kappa^{n+1}-\kappa^{0}\right\|_{C\left([0, T], H^{s+1}\right)} \leq K_{1},\left\|\mathbf{T}^{n}-\mathbf{T}^{0}\right\|_{C\left([0, T], H^{s+1}\right)} \leq K_{2}
$$


then the following estimate holds:

$$
\begin{aligned}
& \left\|T_{\mathbf{n}}^{n+1}-T_{\mathbf{n}}^{0}\right\|_{C\left([0, T], H^{s+1}\right)} \leq \\
& \quad C_{T} C_{2}\left(\left\|\kappa^{n+1}-\kappa^{0}\right\|_{C\left([0, T], H^{s+1}\right)}+\left\|\mathbf{T}^{n}-\mathbf{T}^{0}\right\|_{C\left([0, T], H^{s+1}\right)}+\varphi_{2}\left(\mathbf{T}^{0}\right)\right),
\end{aligned}
$$

where $C_{T}$ is as defined in Lemma 5.3, $C_{2}$ is non-negative constant and $\varphi_{2}\left(\mathbf{T}^{0}\right)$ is a positive constant depending on $\mathbf{T}^{0}$. Additionally $C_{2}$ and $\varphi_{2}\left(\mathbf{T}^{0}\right)$ are both independent of $n$ and $T$.

Proof. We rewrite the problem (5.32) in the form

$$
\begin{array}{lr}
\frac{\partial S_{\mathbf{n}}^{n+1}}{\partial t}+\lambda S_{\mathbf{n}}^{n+1}=g_{\mathbf{n}}\left(\kappa^{n+1}, \mathbf{T}^{n}\right)-g_{\mathbf{n}}\left(\kappa^{0}, \mathbf{T}^{0}\right)+\left(g_{\mathbf{n}}\left(\kappa^{0}, \mathbf{T}^{0}\right)-\lambda T_{\mathbf{n}}^{0}\right) \\
\text { in }(0, h) \times[0, \mathrm{~T}], \\
S_{\mathbf{n}}^{n+1}(y, 0)=0 \quad \text { in }(0, h),
\end{array}
$$

where

$$
S_{\mathbf{n}}^{n+1}(y, t)=T_{\mathbf{n}}^{n+1}(y, t)-T_{\mathbf{n}}^{0}(y) .
$$

Then we proceed as in the proof of Lemma 4.5.

Next, we show that for sufficiently small time interval $[0, \mathrm{~T}]$, the sequence $\left(\kappa^{n}-\kappa^{0}, \mathbf{T}^{n}-\mathbf{T}^{0}\right)$ we have already constructed is bounded independently of $n$ by small bounds in the space $C\left([0, \mathrm{~T}], H^{s+1}(0, h)\right) \times C\left([0, \mathrm{~T}], H^{s+1}(0, h)\right)$.

Lemma 5.5 Let $s$ be an integer $\geq 0$. For $T$ sufficiently small, there exist constants $K_{3}, K_{4}$ independent of $n$ and $T$ such that

$$
\left.\begin{array}{l}
\left\|\mathbf{T}^{n}-\mathbf{T}^{0}\right\|_{C\left([0, T], H^{s+1}\right)} \leq K_{3} C_{T}, \\
\left\|\kappa^{n}-\kappa^{0}\right\|_{C\left([0, T], H^{s+1}\right)} \leq K_{4} C_{T}, \text { for all } n \in \mathbb{N} .
\end{array}\right\}
$$

Recall that $C_{T}=\frac{1-e^{-\lambda T}}{\lambda}$, see formula (5.42).

Proof. Combining the estimates (5.43) and (5.44) with (5.34), we obtain

$$
\left\|\mathbf{T}^{n+1}-\mathbf{T}^{0}\right\|_{C\left([0, \mathrm{~T}], H^{s+1}\right)} \leq C_{\mathrm{T}} C_{3}\left(\left\|\mathbf{T}^{n}-\mathbf{T}^{0}\right\|_{C\left([0, \mathrm{~T}], H^{s+1}\right)}+\varphi_{3}\left(\mathbf{T}^{0}\right)\right),
$$


where $C_{3}$ and $\varphi_{3}\left(\mathbf{T}^{0}\right)$ are non-negative constants independent of $n$ and T.

Now, if we choose $\mathrm{T}$ so that $C_{\mathrm{T}} C_{3} \leq c<1$, then for $K_{3}=\frac{C_{3} \varphi_{3}\left(\mathbf{T}^{0}\right)}{1-c}$ we have

$$
C_{\mathrm{T}} C_{3}\left(K_{3} C_{\mathrm{T}}+\varphi_{3}\left(\mathbf{T}^{0}\right)\right) \leq K_{3} C_{\mathrm{T}}
$$

Thus, by the principle of induction, we see that the sequence $\mathbf{T}^{n}-\mathbf{T}^{0}$ is bounded in the space $C\left([0, \mathrm{~T}], H^{s+1}(0, h)\right)$ by $K_{3} C_{\text {T. }}$. Consequently, according to the formula $(5.34), \kappa^{n}-\kappa^{0}$ should be bounded in the same space by $K_{3} C_{1} C_{\mathrm{T}}$.

\subsection{Convergence of the scheme}

In the following, we will prove the convergence of the scheme we have constructed previously. Let $\left(\tilde{\kappa}^{n}, \tilde{\mathbf{T}}^{n}\right)$ be the sequence obtained by subtracting the solution $\left(\kappa^{n-1}, \mathbf{T}^{n-1}\right)$ of the scheme at iteration $n-1$ from that at iteration $n$

$$
\left(\tilde{\kappa}^{n}, \tilde{\mathbf{T}}^{n}\right)=\left(\kappa^{n}-\kappa^{n-1}, \mathbf{T}^{n}-\mathbf{T}^{n-1}\right) .
$$

Similarly, we define $\tilde{q}^{n}, \tilde{w}^{n}, \tilde{a}_{12}^{n}$ and $\tilde{b}_{12}^{n}$. Now, we subtract the problems $(5.28)_{n-1},(5.30)_{n-1}$, $(5.31)_{n-1}$ and $(5.32)_{n-1}$ from the problems $(5.28)_{n},(5.30)_{n},(5.31)_{n}$ and $(5.32)_{n}$, respectively. The new sequences satisfy the following problems:

$$
\begin{aligned}
& \left.\begin{array}{cc}
-\mu \tilde{\kappa}^{n+1}(y, t)+\tilde{q}^{n+1}(t) y+\tilde{w}^{n+1}(t)=g_{12}\left(\kappa^{n+1}, \mathbf{T}^{n}\right)-g_{12}\left(\kappa^{n}, \mathbf{T}^{n-1}\right) \\
& \text { in }(0, h) \times[0, \mathrm{~T}], \\
\int_{0}^{h} \tilde{\kappa}^{n+1}(y, t) d y=0, \int_{0}^{h} y \tilde{\kappa}^{n+1}(y, t) d y=0 & \text { in }[0, \mathrm{~T}],
\end{array}\right\} \\
& \left.\begin{array}{l}
\frac{d}{d t} \tilde{a}_{12}^{n+1}(t)+\lambda \tilde{a}_{12}^{n+1}(t)=\tilde{q}^{n+1}(t) \quad t \in[0, \mathrm{~T}], \\
\tilde{a}_{12}^{n+1}(0)=0,
\end{array}\right\} \\
& \left.\begin{array}{l}
\frac{d}{d t} \tilde{b}_{12}^{n+1}(t)+\lambda \tilde{b}_{12}^{n+1}(t)=\tilde{w}^{n+1}(t) \quad t \in[0, \mathrm{~T}], \\
\tilde{b}_{12}^{n+1}(0)=0,
\end{array}\right\} \\
& \frac{\partial \tilde{T}_{\mathbf{n}}^{n+1}}{\partial t}(y, t)+\lambda \tilde{T}_{\mathbf{n}}^{n+1}(y, t)=g_{\mathbf{n}}\left(\kappa^{n+1}, \mathbf{T}^{n}\right)-g_{\mathbf{n}}\left(\kappa^{n}, \mathbf{T}^{n-1}\right) \\
& \text { in }(0, h) \times[0, \mathrm{~T}] \text {, } \\
& \tilde{T}_{\mathbf{n}}^{n+1}(y, 0)=0 \quad \text { in }(0, h) .
\end{aligned}
$$


Lemma 5.6 The sequences $\tilde{\kappa}^{n}, \tilde{q}^{n}, \tilde{w}^{n}$ defined above satisfy the following estimates:

$$
\begin{gathered}
\left\|\tilde{q}^{n+1}\right\|_{C([0, T])}+\left\|\tilde{w}^{n+1}\right\|_{C([0, T])} \leq C_{4}\left\|\tilde{\mathbf{T}}^{n}\right\|_{C\left([0, T], H^{s+1}\right)}, \\
\left\|\tilde{\kappa}^{n+1}\right\|_{C\left([0, T], H^{s+1}\right)} \leq C_{5}\left\|\tilde{\mathbf{T}}^{n}\right\|_{C\left([0, T], H^{s+1}\right)},
\end{gathered}
$$

where $C_{4}$ and $C_{5}$ are non-negative constants independent of $n$ and $T$.

Proof. By the mean value theorem, the equation of problem (5.47) can be rewritten as

$$
-\tilde{\kappa}^{n+1}+\tilde{q}^{n+1}(t) y \xi_{c}+\tilde{w}^{n+1}(t) \xi_{c}=\xi_{c} D_{\mathbf{T}} g_{12}\left(\kappa_{c}, \mathbf{T}_{c}\right) \cdot \tilde{\mathbf{T}}^{n}
$$

where

$$
\xi_{c}(y, t)=1 /\left(\mu+\frac{\partial g_{12}}{\partial \kappa}\left(\kappa_{c}(y, t), \mathbf{T}_{c}(y, t)\right)\right)
$$

with

$$
\left\|\kappa_{c}-\kappa^{0}\right\|_{C\left([0, \mathrm{~T}], H^{s+1}\right)} \leq K_{4} C_{\mathrm{T}},\left\|\mathbf{T}_{c}-\mathbf{T}^{0}\right\|_{C\left([0, \mathrm{~T}], H^{s+1}\right)} \leq K_{3} C_{\mathrm{T}}
$$

Now, we integrate the equation (5.53) with respect to $y$ over $(0, h)$, and since $\int_{0}^{h} \tilde{\kappa}^{n+1}(y, t) d y=$ 0 , we obtain

$$
\tilde{q}^{n+1}(t) \int_{0}^{h} y \xi_{c} d y+\tilde{w}^{n+1}(t) \int_{0}^{h} \xi_{c} d y=\int_{0}^{h} \xi_{c} D_{\mathbf{T}} g_{12}\left(\kappa_{c}, \mathbf{T}_{c}\right) \cdot \tilde{\mathbf{T}}^{n} d y .
$$

Similarly, we multiply (5.53) by $y$ then integrate, thus yielding

$$
\tilde{q}^{n+1}(t) \int_{0}^{h} y^{2} \xi_{c} d y+\tilde{w}^{n+1}(t) \int_{0}^{h} y \xi_{c} d y=\int_{0}^{h} y \xi_{c} D_{\mathbf{T}} g_{12}\left(\kappa_{c}, \mathbf{T}_{c}\right) \cdot \tilde{\mathbf{T}}^{n} d y .
$$

Since $g_{12}$ satisfies the condition (5.18), then if we proceed as in the proof of Lemma 5.1 when rewriting the Jacobian determinant as in formula (5.24), there exists a constant $a_{3}>0$ such that

$$
0<-1 /\left(\left(\int_{0}^{h} y \xi_{c} d y\right)^{2}-\left(\int_{0}^{h} y^{2} \xi_{c} d y\right)\left(\int_{0}^{h} \xi_{c} d y\right)\right) \leq a_{3} .
$$

Thus, from the system of equations (5.54) and (5.55), $\left(\tilde{q}^{n+1}(t), \tilde{w}^{n+1}(t)\right)$ can be expressed in terms of $\tilde{\mathbf{T}}^{n}$ and satisfy the following estimates for some constant $C>0$ independent of $n$ and $\mathrm{T}$.

$$
\left\|\tilde{q}^{n+1}\right\|_{C([0, T])} \leq C\left\|\tilde{\mathbf{T}}^{n}\right\|_{C\left([0, T], H^{s+1}\right)}, \quad\left\|\tilde{w}^{n+1}\right\|_{C([0, \mathrm{~T}])} \leq C\left\|\tilde{\mathbf{T}}^{n}\right\|_{C\left([0, \mathrm{~T}], H^{s+1}\right)} .
$$


Consequently, the estimate (5.52) can be easily obtained from (5.53).

Now, we apply the estimates obtained in Lemmas 5.3, 5.4 and 5.5 to the problems (5.48) (5.49) and (5.50) to deduce the following estimate:

$$
\left\|\tilde{\mathbf{T}}^{n+1}\right\|_{C\left([0, \mathrm{~T}], H^{s+1}\right)} \leq C_{\mathrm{T}} C_{6}\left\|\tilde{\mathbf{T}}^{n}\right\|_{C\left([0, \mathrm{~T}], H^{s+1}\right)},
$$

for some non-negative constant $C_{6}$ independent of $n$ and т. Recall that $C_{\mathrm{T}}=\frac{1-e^{-\lambda \mathrm{T}}}{\lambda}$.

If $\mathrm{T}$ is chosen sufficiently small so that $C_{\mathrm{T}} C_{6}<1$, then from (5.52) and (5.56) we conclude that the sequence $\left(\kappa^{n}, \mathbf{T}^{n}\right)$ converges in the space

$$
C\left([0, \mathrm{~T}], H^{s+1}\right) \times C\left([0, \mathrm{~T}], H^{s+1}\right) .
$$

As an immediate consequence of the above results, we have the following theorem.

Theorem 5.7 Let $s$ be an integer $\geq 0$ and let $T>0$ be chosen sufficiently small. If $T_{\mathbf{n}}^{0} \in$ $H^{s+1}(0, h)$, then the problem (5.6)-(5.10) has a unique solution

$$
(u, \mathbf{T}) \in C\left([0, T], H^{s+2}\right) \times C\left([0, T], H^{s+1}\right)
$$

which can be obtained by the iterative scheme introduced previously. 


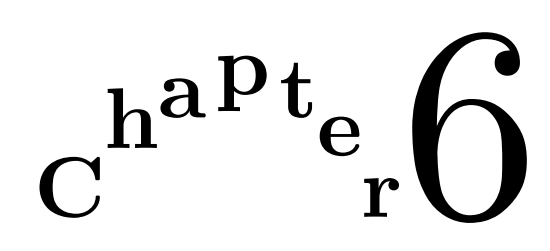

\section{Extension to some three-dimensional flows with large initial data}

\section{Contents}

6.1 Assumptions on the initial data $T_{0} \ldots \ldots \ldots 6$

6.1.1 Johnson-Segalman model with constant initial data $\mathbf{T}_{0} \ldots \ldots 6$

6.2 'Modified' Stokes problem .................. 69

6.3 Problem reformulation ................... 70

6.3.1 Reduction to iterative subproblems . . . . . . . . . . . . 72

6.4 Construction of the solution ............... 73

6.4 .1 Existence .......................... 73

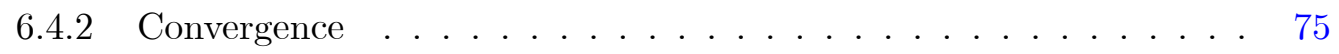

In this chapter, we present some results of existence and uniqueness for three-dimensional flow with arbitrary initial data. The initial data $\mathbf{T}_{0}$ and the body force $\mathbf{f}$ that acting on the fluid are, respectively, functions in the spaces $H^{s+1}$ and $C_{b}\left([0, \mathrm{~T}] ; H^{s+1}\right) \cap C^{1}\left([0, \mathrm{~T}] ; H^{s}\right)$, 
$s \geq 1$, T sufficiently small. The norms $\left\|\mathbf{T}_{0}\right\|_{H^{s+1}},\|\mathbf{f}\|_{C_{b}\left([0, T] ; H^{s+1}\right)}$ and $\left\|\frac{\partial}{\partial t} \mathbf{f}\right\|_{C_{b}\left([0, T] ; H^{s}\right)}$ are not assumed to be small. The function $g$ is assumed to be of the form

$$
g(\nabla V, \mathbf{T})=g_{1}(\nabla V, \mathbf{T})+g_{2}(\mathbf{T})
$$

where $g_{1}(\nabla V, \mathbf{T})$ is linear with respect to both $\nabla V$ and $\mathbf{T}$, i.e. bilinear, and satisfies the estimate

$$
\left\|g_{1}(\nabla V, \mathbf{T})\right\|_{H^{s+1}} \leq C\|V\|_{H^{s+2}}\|\mathbf{T}\|_{H^{s+1}}, C>0, s \geq 1,
$$

and $g_{2}(\mathbf{T})$ is quadratic functions of $\mathbf{T}$, and satisfy the estimate

$$
\left\|g_{2}(\mathbf{T})\right\|_{H^{s+1}} \leq C\|\mathbf{T}\|_{H^{s+1}}^{2}, C>0, s \geq 1
$$

\subsection{Assumptions on the initial data $\mathbf{T}_{0}$}

Let $\mathbb{V}$ be the space of divergence free functions in $H_{0}^{1}(\Omega)^{3}$

$$
\mathbb{V}=\left\{V \in H_{0}^{1}(\Omega)^{3} ; \operatorname{div} V=0\right\}
$$

We define the real valued function $F$ in the space $H_{0}^{1}(\Omega)^{3}$ by

$$
F(V)=\frac{\sum_{i, j=1}^{3} \int_{\Omega}\left[g_{1}\left(\nabla V, \mathbf{T}_{0}\right)-(V \cdot \nabla) \mathbf{T}_{0}\right]_{i j} \frac{\partial V_{i}}{\partial x_{j}} d \mathbf{x}}{\|\nabla V\|_{L^{2}}^{2}} \text { if } V \neq 0 .
$$

Let $\theta_{m}$ and $\theta_{M}$ be, respectively, the infimum and the supremum of $\mu+F$ over $\mathbb{V} /\{0\}$

$$
\theta_{m}=\mu+\inf _{V \in \mathbb{V} /\{0\}} F(V), \theta_{M}=\mu+\sup _{V \in \mathbb{V} /\{0\}} F(V)
$$

By the Poincaré inequality, the function $F$ is bounded. Hence, $\theta_{m}$ and $\theta_{M}$ are finite numbers. We will make the following assumption on the initial data $\mathbf{T}_{0}$ :

$$
0 \notin\left[\theta_{m}, \theta_{M}\right] \text { i.e. either } \theta_{m}>0 \text { or } \theta_{M}<0 \text {. }
$$

A simple case where this assumption is satisfied, when $\mathbf{T}_{0}$ is a constant function and the operator $-\mu \Delta V-\operatorname{div}\left(g_{1}\left(\nabla V, \mathbf{T}_{0}\right)\right)$ is uniformly elliptic. Now, we will give a characterization of the assumption (6.3) for Johnson-Segalman model. 


\subsubsection{Johnson-Segalman model with constant initial data $\mathbf{T}_{0}$}

The function $g_{1}$ for Johnson-Segalman model is

$$
g_{1}(\nabla V, \mathbf{T})=\left(1-\frac{\nu}{2}\right)(\nabla V) \mathbf{T}-\frac{\nu}{2}(\nabla V)^{\mathrm{T}} \mathbf{T}+\left(1-\frac{\nu}{2}\right) \mathbf{T}(\nabla V)^{\mathrm{T}}-\frac{\nu}{2} \mathbf{T}(\nabla V) .
$$

Proposition 6.1 Let $\Lambda_{0 i}, i=1,2,3$ be the eigenvalues of the initial extra stress tensor $\mathbf{T}_{0}$. Let $\alpha_{m}$ and $\alpha_{M}$ be defined by

$$
\begin{aligned}
\alpha_{m} & =\mu+\min _{1 \leq i, j \leq 3}\left\{-\frac{\nu}{2} \Lambda_{0 i}+\left(1-\frac{\nu}{2}\right) \Lambda_{0 j}\right\}, \\
\alpha_{M} & =\mu+\max _{1 \leq i, j \leq 3}\left\{-\frac{\nu}{2} \Lambda_{0 i}+\left(1-\frac{\nu}{2}\right) \Lambda_{0 j}\right\} .
\end{aligned}
$$

If $\alpha_{m}>0$ or $\alpha_{M}<0$, then the assumption (6.3) is satisfied.

We remark that only the case $\alpha_{m}>0$ is physically relevant.

First, we show that

$$
\int_{\Omega}\left(\operatorname{div}\left[(\nabla V)^{\mathrm{T}} \mathbf{T}_{0}\right]\right) \cdot V d \mathbf{x}=0, \int_{\Omega}\left(\operatorname{div}\left[\mathbf{T}_{0}(\nabla V)^{\mathrm{T}}\right]\right) \cdot V d \mathbf{x}=0 .
$$

By applying the divergence theorem, we have

$$
\begin{aligned}
& \int_{\Omega}(\operatorname{div} {\left.\left[(\nabla V)^{\mathrm{T}} \mathbf{T}_{0}\right]\right) \cdot V d \mathbf{x}=} \\
& \int_{\partial \Omega}\left(V^{\mathrm{T}}(\nabla V)^{\mathrm{T}} \mathbf{T}_{0}\right) \cdot n d S-\sum_{i, j=1}^{3} \int_{\Omega}\left[(\nabla V)^{\mathrm{T}} \mathbf{T}_{0}\right]_{i j} \frac{\partial V_{i}}{\partial x_{j}} d \mathbf{x},
\end{aligned}
$$

where $n$ is the unit outward normal vector of the surface $\partial \Omega$ and $d S$ is the surface area element. Then, since $V=0$ on $\partial \Omega$, we obtain

$$
\begin{aligned}
\int_{\Omega}\left(\operatorname{div}\left[(\nabla V)^{\mathrm{T}} \mathbf{T}_{0}\right]\right) \cdot V d \mathbf{x} & =-\sum_{i, j=1}^{3} \int_{\Omega}\left[(\nabla V)^{\mathrm{T}} \mathbf{T}_{0}\right]_{i j} \frac{\partial V_{i}}{\partial x_{j}} d \mathbf{x} \\
& =\sum_{i, j, k=1}^{3} \int_{\Omega} \frac{\partial V_{k}}{\partial x_{i}} \mathbf{T}_{0 k j} \frac{\partial V_{i}}{\partial x_{j}} d \mathbf{x} .
\end{aligned}
$$


For constant initial data $\mathbf{T}_{0}$, we have

$$
\begin{aligned}
\sum_{i, j, k=1}^{3} \int_{\Omega} \frac{\partial V_{k}}{\partial x_{i}} \mathbf{T}_{0 k j} \frac{\partial V_{i}}{\partial x_{j}} d \mathbf{x} & =\sum_{j, k=1}^{3} \mathbf{T}_{0 k j} \int_{\Omega} \sum_{i=1}^{3} \frac{\partial V_{k}}{\partial x_{i}} \frac{\partial V_{i}}{\partial x_{j}} d \mathbf{x} \\
& =\sum_{j, k=1}^{3} \mathbf{T}_{0 k j} \int_{\Omega}\left(\nabla V_{k}\right) \cdot \frac{\partial V}{\partial x_{j}} d \mathbf{x}
\end{aligned}
$$

Again, by applying the divergence theorem

$$
\int_{\Omega}\left(\nabla V_{k}\right) \cdot \frac{\partial V}{\partial x_{j}} d \mathbf{x}=\int_{\partial \Omega} V_{k} \frac{\partial V}{\partial x_{j}} \cdot n d S-\int_{\Omega} V_{k} \frac{\partial(\operatorname{div} V)}{\partial x_{j}} d \mathbf{x} .
$$

Since $\operatorname{div} V=0$ in $\Omega$ and $V=0$ on $\partial \Omega$, then by combining the above equations we obtain

$$
\int_{\Omega}\left(\operatorname{div}\left[(\nabla V)^{\mathrm{T}} \mathbf{T}_{0}\right]\right) \cdot V d \mathbf{x}=0 .
$$

Similarly, we prove the second equality of the equation (6.5). Thus

$$
\begin{aligned}
\int_{\Omega}[- & \left.\operatorname{div}\left(g_{1}\left(\nabla V, \mathbf{T}_{0}\right)\right)\right] \cdot V d \mathbf{x}= \\
& \quad-\int_{\Omega}\left(\operatorname{div}\left[\left(1-\frac{\nu}{2}\right)(\nabla V) \mathbf{T}_{0}-\frac{\nu}{2} \mathbf{T}_{0}(\nabla V)\right]\right) \cdot V d \mathbf{x} .
\end{aligned}
$$

By spectral decomposition, the symmetric tensor $\mathbf{T}_{0}$ can be decomposed into

$$
\mathbf{T}_{0}=\mathbf{S}_{0} \Lambda_{0} \mathbf{S}_{0}^{\mathrm{T}}
$$

where $\mathbf{S}_{0}$ is an orthogonal $3 \times 3$ matrix and $\Lambda_{0}$ is a $3 \times 3$ diagonal matrix formed from the eigenvalues of $\mathbf{T}_{0}$.

Using the properties of the divergence and gradient operators, we easily show the following identities:

$$
\begin{aligned}
\left(\operatorname{div}\left[(\nabla V) \mathbf{T}_{0}\right]\right) \cdot V & =\left(\operatorname{div}\left[\left(\nabla\left(\mathbf{S}_{0}^{\mathrm{T}} V\right)\right) \mathbf{T}_{0}\right]\right) \cdot\left(\mathbf{S}_{0}^{\mathrm{T}} V\right), \\
\left(\operatorname{div}\left[\mathbf{T}_{0}(\nabla V)\right]\right) \cdot V & =\left(\operatorname{div}\left[\Lambda_{0}\left(\nabla\left(\mathbf{S}_{0}^{\mathrm{T}} V\right)\right)\right]\right) \cdot\left(\mathbf{S}_{0}^{\mathrm{T}} V\right), \\
(\operatorname{div}[\nabla V]) \cdot V & =\left(\operatorname{div}\left[\nabla\left(\mathbf{S}_{0}^{\mathrm{T}} V\right)\right]\right) \cdot\left(\mathbf{S}_{0}^{\mathrm{T}} V\right) .
\end{aligned}
$$

Combining the identities (6.8), (6.9) and (6.10) with the equation (6.6), we obtain

$$
\begin{aligned}
\int_{\Omega}[- & \left.\operatorname{div}\left(g_{1}\left(\nabla V, \mathbf{T}_{0}\right)\right)\right] \cdot V d \mathbf{x}= \\
& \quad-\int_{\Omega}\left(\operatorname{div}\left[-\frac{\nu}{2} \Lambda_{0}(\nabla W)+\left(1-\frac{\nu}{2}\right)(\nabla W) \mathbf{T}_{0}\right]\right) \cdot W d \mathbf{x},
\end{aligned}
$$


where

$$
W=\mathbf{S}_{0}^{\mathrm{T}} V
$$

Since $V=0$ on $\partial \Omega$, so is $W$. Then by applying the divergence theorem

$$
\begin{aligned}
-\int_{\Omega}\left(\operatorname{div}\left[-\frac{\nu}{2} \Lambda_{0}(\nabla W)\right]\right) \cdot W d \mathbf{x} & =\sum_{i, j=1}^{3} \int_{\Omega}\left[-\frac{\nu}{2} \Lambda_{0}(\nabla W)\right]_{i j} \frac{\partial W_{i}}{\partial x_{j}} d \mathbf{x} \\
& =\sum_{i, j, k=1}^{3} \int_{\Omega}\left(-\frac{\nu}{2} \Lambda_{0}\right)_{i k} \frac{\partial W_{k}}{\partial x_{j}} \frac{\partial W_{i}}{\partial x_{j}} d \mathbf{x}
\end{aligned}
$$

which can be rewritten as

$$
-\int_{\Omega}\left(\operatorname{div}\left[-\frac{\nu}{2} \Lambda_{0}(\nabla W)\right]\right) \cdot W d \mathbf{x}=\sum_{i=1}^{3} \int_{\Omega}\left(\nabla W_{i}\right)^{\mathrm{T}}\left(-\frac{\nu}{2} \Lambda_{0 i}\right)\left(\nabla W_{i}\right) d \mathbf{x} .
$$

Similarly we obtain

$$
-\int_{\Omega}\left(\operatorname{div}\left[(\nabla W) \mathbf{T}_{0}\right]\right) \cdot W d \mathbf{x}=\sum_{i=1}^{3} \int_{\Omega}\left(\nabla W_{i}\right)^{\mathrm{T}} \mathbf{T}_{0}\left(\nabla W_{i}\right) d \mathbf{x} .
$$

Combining the two formulas above, the equation (6.11) becomes

$$
\begin{aligned}
& \int_{\Omega}\left[-\operatorname{div}\left(g_{1}\left(\nabla V, \mathbf{T}_{0}\right)\right)\right] \cdot V d \mathbf{x}= \\
& \quad \sum_{i=1}^{3} \int_{\Omega}\left(\nabla W_{i}\right)^{\mathrm{T}}\left[-\frac{\nu}{2} \Lambda_{0 i} I+\left(1-\frac{\nu}{2}\right) \mathbf{T}_{0}\right]\left(\nabla W_{i}\right) d \mathbf{x},
\end{aligned}
$$

where $I$ is the $3 \times 3$ identity matrix. Again, using spectral decomposition $\mathbf{T}_{0}=\mathbf{S}_{0} \Lambda_{0} \mathbf{S}_{0}^{\mathrm{T}}$, we see that

$$
\begin{aligned}
& \sum_{i=1}^{3}\left(\nabla W_{i}\right)^{\mathrm{T}}\left[-\frac{\nu}{2} \Lambda_{0 i} I+\left(1-\frac{\nu}{2}\right) \mathbf{T}_{0}\right]\left(\nabla W_{i}\right)= \\
& \sum_{i=1}^{3}\left(\mathbf{S}_{0}^{\mathrm{T}} \nabla W_{i}\right)^{\mathrm{T}}\left[-\frac{\nu}{2} \Lambda_{0 i} I+\left(1-\frac{\nu}{2}\right) \Lambda_{0}\right]\left(\mathbf{S}_{0}^{\mathrm{T}} \nabla W_{i}\right) .
\end{aligned}
$$

Since $\left(\mathbf{S}_{0}^{\mathrm{T}} \nabla W_{i}\right)_{j}=\left(\mathbf{S}_{0}^{\mathrm{T}}(\nabla V) \mathbf{S}_{0}\right)_{i j}$, the formula (6.15) can be rewritten as

$$
\int_{\Omega}\left[-\operatorname{div}\left(g_{1}\left(\nabla V, \mathbf{T}_{0}\right)\right)\right] \cdot V d \mathbf{x}=\sum_{i, j=1}^{3}\left[-\frac{\nu}{2} \Lambda_{0 i}+\left(1-\frac{\nu}{2}\right) \Lambda_{0 j}\right] \int_{\Omega}\left(U_{i j}\right)^{2} d \mathbf{x},
$$

where

$$
U=\mathbf{S}_{0}^{\mathrm{T}}(\nabla V) \mathbf{S}_{0} .
$$


Applying the divergence theorem to left-hand side of the formula (6.16) and since $\|\nabla V\|_{L^{2}}=$ $\|U\|_{L^{2}}$, the functional $F$ defined on (6.1) can be given simply by the following expression:

$$
F(V)=\frac{\sum_{i, j=1}^{3}\left[-\frac{\nu}{2} \Lambda_{0 i}+\left(1-\frac{\nu}{2}\right) \Lambda_{0 j}\right] \int_{\Omega}\left(U_{i j}\right)^{2} d \mathbf{x}}{\|U\|_{L^{2}}^{2}} .
$$

Therefore, if $\alpha_{m}>0$ or $\alpha_{M}<0$, then the assumption (6.3) is satisfied, and this completes the proof of Proposition (6.1).

\section{Upper Convected Maxwell model (UCM)}

The function $g_{1}$ for UCM model is $(\nu=0)$

$$
g_{1}(\nabla V, \mathbf{T})=(\nabla V) \mathbf{T}+\mathbf{T}(\nabla V)^{\mathrm{T}} .
$$

For UCM model the assumption (6.3) is satisfied if either $\mu+\min _{1 \leq i \leq 3}\left\{\Lambda_{0 i}\right\}>0$ or $\mu+$ $\max _{1 \leq i \leq 3}\left\{\Lambda_{0 i}\right\}<0$.

\section{Lower Convected Maxwell model (LCM)}

The function $g_{1}$ for LCM model is $(\nu=2)$

$$
g_{1}(\nabla V, \mathbf{T})=-(\nabla V)^{\mathrm{T}} \mathbf{T}-\mathbf{T}(\nabla V) .
$$

For LCM model the assumption (6.3) is satisfied if either $\mu-\max _{1 \leq i \leq 3}\left\{\Lambda_{0 i}\right\}>0$ or $\mu-$ $\min _{1 \leq i \leq 3}\left\{\Lambda_{0 i}\right\}<0$.

\section{2 'Modified' Stokes problem}

We consider the following 'modified' Stokes problem:

$$
\begin{aligned}
& -\mu \Delta V-\operatorname{div}\left(g_{1}\left(\nabla V, \mathbf{T}_{0}\right)\right)+\operatorname{div}\left((V \cdot \nabla) \mathbf{T}_{0}\right)+\nabla q=f \quad \text { in } \Omega, \\
& \operatorname{div} V=0 \quad \text { in } \Omega \\
& V=0 \quad \text { on } \partial \Omega .
\end{aligned}
$$


Proposition 6.2 If the assumption (6.3) is satisfied, then for $f \in H^{s}(\Omega), s \geq 1$, the problem (6.17)-(6.19) has a unique solution $(V, q)$ in the space

$$
\left(\mathbb{V} \cap H^{s+2}(\Omega)^{3}\right) \times\left(L_{0}^{2}(\Omega) \cap H^{s+1}(\Omega)\right),
$$

and this solution satisfies the estimate

$$
\|V\|_{H^{s+2}}+\|q\|_{H^{s+1}} \leq C\|f\|_{H^{s}}, \quad C>0 .
$$

Proof. Under the assumption (6.3), the bilinear mapping that represents the variational formulation of the problem (6.17)-(6.19) satisfies the coercivity condition of Lax-Milgram lemma. The rest of the proof is similar to that of Stokes problem. For example, see [32, Chapter 1, Section 2].

\subsection{Problem reformulation}

We use the method introduced by Renardy in [26] to split the problem (1.12)-(1.16) into three subproblems. First, we define

$$
(\tilde{\mathbf{T}}, \tilde{p}, \tilde{\mathbf{f}}) \equiv\left(\mathbf{T}-\mathbf{T}_{0}, p-p_{0}, \mathbf{f}-\mathbf{f}_{0}\right),
$$

where $\mathbf{f}_{0}$ and $p_{0}$ denote respectively the values of $\mathbf{f}$ and $p$ at $t=0$. With the new functions $\tilde{\mathbf{T}}, \tilde{p}$ and $\tilde{\mathbf{f}}$ introduced above, the equations (1.12), (1.15) and (1.16) become

$$
\begin{aligned}
& \operatorname{div} \tilde{\mathbf{T}}-\nabla \tilde{p}=\tilde{\mathbf{f}} \quad \text { in } \Omega \times[0, \mathrm{~T}], \\
& \frac{\partial \tilde{\mathbf{T}}}{\partial t}+(V \cdot \nabla) \tilde{\mathbf{T}}+\lambda \tilde{\mathbf{T}}=\mu\left(\nabla V+(\nabla V)^{\mathrm{T}}\right)-(V \cdot \nabla) \mathbf{T}_{0}-\lambda \mathbf{T}_{0} \\
& +g\left(\nabla V, \tilde{\mathbf{T}}+\mathbf{T}_{0}\right) \text { in } \Omega \times[0, \mathrm{~T}], \\
& \tilde{\mathbf{T}}(\cdot, 0)=0 \quad \text { in } \Omega \text {. }
\end{aligned}
$$

Next, by applying the divergence operator to both sides of the equation (6.23), we obtain

$$
\begin{aligned}
& \frac{\partial}{\partial t} \operatorname{div} \tilde{\mathbf{T}}+(V \cdot \nabla) \operatorname{div} \tilde{\mathbf{T}}+\sum_{j=1}^{3}\left(\frac{\partial V}{\partial x_{j}} \cdot \nabla\right) \tilde{\mathbf{T}}_{i j}+\lambda \operatorname{div} \tilde{\mathbf{T}}=-\lambda \operatorname{div} \mathbf{T}_{\mathbf{0}} \\
& \quad+\mu(\Delta V+\nabla(\operatorname{div} V))-\operatorname{div}\left((V \cdot \nabla) \mathbf{T}_{0}\right)+\operatorname{div}\left(g\left(\nabla V, \tilde{\mathbf{T}}+\mathbf{T}_{0}\right)\right) .
\end{aligned}
$$


We use the equations (6.22) and (1.13) to replace $\operatorname{div} \tilde{\mathbf{T}}$ and $\operatorname{div} V$, respectively, by $\nabla \tilde{p}+\tilde{\mathbf{f}}$ and 0 . In the equation obtained, we substitute $(V \cdot \nabla) \nabla \tilde{p}$ using the identity

$$
(V \cdot \nabla) \nabla \tilde{p}=\nabla((V \cdot \nabla) \tilde{p})-(\nabla V)^{\mathrm{T}} \nabla \tilde{p}
$$

Thus, the equation (6.25) becomes

$$
\begin{array}{r}
-\mu \Delta V-\operatorname{div}\left(g_{1}\left(\nabla V, \mathbf{T}_{0}\right)\right)+\operatorname{div}\left((V \cdot \nabla) \mathbf{T}_{0}\right)+\nabla q \\
=(\nabla V)^{\mathrm{T}} \nabla \tilde{p}-\sum_{j=1}^{3}\left(\frac{\partial V}{\partial x_{j}} \cdot \nabla\right) \tilde{\mathbf{T}}_{i j}-(V \cdot \nabla) \tilde{\mathbf{f}}-\left(\frac{\partial}{\partial t} \tilde{\mathbf{f}}+\lambda \tilde{\mathbf{f}}\right) \\
\quad-\lambda \operatorname{div} \mathbf{T}_{0}+\operatorname{div}\left[g_{1}(\nabla V, \tilde{\mathbf{T}})+g_{2}\left(\tilde{\mathbf{T}}+\mathbf{T}_{0}\right)\right],
\end{array}
$$

where

$$
q=\frac{\partial}{\partial t} \tilde{p}+(V \cdot \nabla) \tilde{p}+\lambda \tilde{p}
$$

If the assumption (6.3) holds, then by Proposition 6.2 there exists a unique

$$
\left(V^{0}, q^{0}\right) \in\left(\mathbb{V} \cap H^{s+2}(\Omega)^{3}\right) \times\left(L_{0}^{2}(\Omega) \cap H^{s+1}(\Omega)\right),
$$

satisfying the following equation:

$$
\begin{aligned}
-\mu \Delta V^{0}-\operatorname{div}\left(g_{1}\left(\nabla V^{0}, \mathbf{T}_{0}\right)\right)+\operatorname{div}\left(\left(V^{0} \cdot \nabla\right) \mathbf{T}_{0}\right)+\nabla q^{0} \\
=-\left(\frac{\partial}{\partial t} \tilde{\mathbf{f}}+\lambda \tilde{\mathbf{f}}\right)-\lambda \operatorname{div} \mathbf{T}_{0}+\operatorname{div}\left(g_{2}\left(\mathbf{T}_{0}\right)\right) .
\end{aligned}
$$

Now, we define $(\tilde{V}, \tilde{q}) \equiv\left(V-V^{0}, q-q^{0}\right)$. Subtracting the equation (6.28) from (6.26), we see that $\tilde{V}$ and $\tilde{q}$ satisfy

$$
\begin{array}{r}
-\mu \Delta \tilde{V}-\operatorname{div}\left(g_{1}\left(\nabla \tilde{V}, \mathbf{T}_{0}\right)\right)+\operatorname{div}\left((\tilde{V} \cdot \nabla) \mathbf{T}_{0}\right)+\nabla \tilde{q}=(\nabla V)^{\mathrm{T}} \nabla \tilde{p}-(V \cdot \nabla) \tilde{\mathbf{f}} \\
-\sum_{j=1}^{3}\left(\frac{\partial V}{\partial x_{j}} \cdot \nabla\right) \tilde{\mathbf{T}}_{i j}+\operatorname{div}\left(g_{1}(\nabla V, \tilde{\mathbf{T}})\right)+\operatorname{div}\left[g_{2}\left(\tilde{\mathbf{T}}+\mathbf{T}_{0}\right)-g_{2}\left(\mathbf{T}_{0}\right)\right] .
\end{array}
$$

The problem (1.12)-(1.16) can be reformulated as follows: Find a vector field $\tilde{V}$, a tensor $\tilde{\mathbf{T}}$ and two scalar functions $\tilde{p}$ and $\tilde{q}$ satisfying the following subproblems:

$$
\left.\begin{array}{ll}
\frac{\partial \tilde{p}}{\partial t}+(V \cdot \nabla) \tilde{p}+\lambda \tilde{p}=\tilde{q}+q^{0} & \text { in } \Omega \times[0, \mathrm{~T}], \\
\tilde{p}(\cdot, 0)=0 & \text { in } \Omega .
\end{array}\right\}
$$




$$
\begin{aligned}
& \left.\begin{array}{l}
\text { Equation }(6.29), \\
\operatorname{div} \tilde{V}=0 \quad \text { in } \Omega \times[0, \mathrm{~T}], \\
\tilde{V}=0 \quad \text { on } \partial \Omega \times[0, \mathrm{~T}], \\
V=\tilde{V}+V^{0} . \\
\quad \text { Equation }(6.23), \\
\tilde{\mathbf{T}}(\cdot, 0)=0 \quad \text { in } \Omega .
\end{array}\right\}
\end{aligned}
$$

\subsubsection{Reduction to iterative subproblems}

As in the last two chapters, we transform our problem to iterative linear subproblems. Then, we construct a sequence of solutions for those iterative subproblems, converging to a solution for our original problem.

At $t=0$ the value of all the quantities $\tilde{\mathbf{T}}, \tilde{V}, \tilde{p}$ and $\tilde{q}$ is zero. So, it is obvious to initialize the scheme by choosing

$$
\tilde{\mathbf{T}}^{0}=0, \tilde{V}^{0}=0, \tilde{p}^{0}=0, \tilde{q}^{0}=0 .
$$

In subsequent iterations, we solve successively the following 'modified' Stokes problem for $\tilde{V}^{n+1}$ and $\tilde{q}^{n+1}$ :

$$
\begin{aligned}
& -\mu \Delta \tilde{V}^{n+1}-\operatorname{div}\left(g_{1}\left(\nabla \tilde{V}^{n+1}, \mathbf{T}_{0}\right)\right)+\operatorname{div}\left(\left(\tilde{V}^{n+1} \cdot \nabla\right) \mathbf{T}_{0}\right)+\nabla \tilde{q}^{n+1} \\
& =\left(\nabla V^{n}\right)^{\mathrm{T}}\left(\operatorname{div} \tilde{\mathbf{T}}^{n}-\tilde{\mathbf{f}}\right)-\left(V^{n} \cdot \nabla\right) \tilde{\mathbf{f}}-\sum_{j=1}^{3}\left(\frac{\partial V^{n}}{\partial x_{j}} \cdot \nabla\right) \tilde{\mathbf{T}}_{i j}^{n} \\
& \quad+\operatorname{div}\left[g_{1}\left(\nabla V^{n}, \tilde{\mathbf{T}}^{n}\right)+g_{2}\left(\tilde{\mathbf{T}}^{n}+\mathbf{T}_{0}\right)-g_{2}\left(\mathbf{T}_{0}\right)\right] \text { in } \Omega \times[0, \mathrm{~T}] \\
& \operatorname{div} \tilde{V}^{n+1}=0 \quad \operatorname{in} \Omega \times[0, \mathrm{~T}] \\
& \tilde{V}^{n+1}=0 \quad \text { on } \partial \Omega \times[0, \mathrm{~T}] \\
& V^{n}=\tilde{V}^{n}+V^{0} .
\end{aligned}
$$


Also, we solve two evolution problems, one for $\tilde{\mathbf{T}}^{n+1}$

$$
\begin{aligned}
& \frac{\partial \tilde{\mathbf{T}}^{n+1}}{\partial t}+\left(V^{n+1} \cdot \nabla\right) \tilde{\mathbf{T}}^{n+1}+\lambda \tilde{\mathbf{T}}^{n+1}=\mu\left(\nabla V^{n+1}+\left(\nabla V^{n+1}\right)^{\mathrm{T}}\right)-\lambda \mathbf{T}_{0} \\
& \quad-\left(V^{n+1} \cdot \nabla\right) \mathbf{T}_{0}+g_{1}\left(\nabla V^{n+1}, \tilde{\mathbf{T}}^{n}+\mathbf{T}_{0}\right)+g_{2}\left(\tilde{\mathbf{T}}^{n}+\mathbf{T}_{0}\right) \text { in } \Omega \times[0, \mathrm{~T}], \\
& V^{n+1}=\tilde{V}^{n+1}+V^{0}, \\
& \tilde{\mathbf{T}}^{n+1}(\cdot, 0)=0 \quad \text { in } \Omega,
\end{aligned}
$$

and another one for $\tilde{p}^{n+1}$

$$
\begin{aligned}
& \frac{\partial \tilde{p}^{n+1}}{\partial t}+\left(\left(\tilde{V}^{n}+V^{0}\right) \cdot \nabla\right) \tilde{p}^{n+1}+\lambda \tilde{p}^{n+1}=\tilde{q}^{n+1}+q^{0} \quad \text { in } \Omega \times[0, \mathrm{~T}], \\
& \tilde{p}^{n+1}(\cdot, 0)=0 \quad \text { in } \Omega .
\end{aligned}
$$

\subsection{Construction of the solution}

In this section, we show that the problem (1.12)-(1.16) has a unique local solution in time which can be constructed from the iterative scheme (6.33)-(6.35).

\subsubsection{Existence}

In the following three lemmas, we present results of existence and uniqueness for the iterative subproblems (6.33)-(6.35). We also give some estimates that will be useful in the proof of convergence.

Lemma 6.3 If $\tilde{\mathbf{f}}, \tilde{\mathbf{T}}^{n} \in C_{b}\left(0, T ; H^{s+1}(\Omega)\right)$ and $\tilde{V}^{n} \in C_{b}\left(0, T ; H^{s+2}(\Omega)\right)$, then the problem (6.33) has a unique solution

$$
\left(\tilde{V}^{n+1}, \tilde{q}^{n+1}\right) \in C_{b}\left(0, T ; H^{s+2}(\Omega)\right) \times C_{b}\left(0, T ; H^{s+1}(\Omega) \cap L_{0}^{2}(\Omega)\right) .
$$

Moreover, for small time interval $[0, T]$, there exist $\varepsilon_{1}, \varepsilon_{2}>0$ such that the following estimate holds if $\left\|\tilde{V}^{n}\right\|_{C_{b}\left(0, T ; H^{s+2}\right)} \leq \varepsilon_{1}$ and $\left\|\tilde{\mathbf{T}}^{n}\right\|_{C_{b}\left(0, T ; H^{s+1}\right)} \leq \varepsilon_{2}$ :

$$
\left\|\tilde{V}^{n+1}\right\|_{C_{b}\left(0, T ; H^{s+2}\right)}+\left\|\tilde{q}^{n+1}\right\|_{C_{b}\left(0, T ; H^{s+1}\right)} \leq C_{1}\left(\|\tilde{\mathbf{f}}\|_{C_{b}\left(0, T ; H^{s+1}\right)}+\left\|\tilde{\mathbf{T}}^{n}\right\|_{C_{b}\left(0, T ; H^{s+1}\right)}\right),
$$


where $C_{1}$ is a non-negative constant independent of $n$.

The proof of this lemma is an application of Proposition 6.2.

Lemma 6.4 Assume that $\tilde{\mathbf{T}}^{n} \in C_{b}\left(0, T ; H^{s+1}(\Omega)\right)$ and $\tilde{V}^{n+1} \in C_{b}\left(0, T ; H^{s+2}(\Omega)\right)$ with $\operatorname{div} \tilde{V}^{n+1}(t)=0$ in $\Omega, \tilde{V}^{n+1}(t)=0$ on $\partial \Omega$ and $\left\|\tilde{V}^{n+1}\right\|_{C_{b}\left(0, T ; H^{s+2}\right)} \leq \varepsilon_{1}, \quad \varepsilon_{1}>0$.

Then there exists a unique solution $\tilde{\mathbf{T}}^{n+1} \in C_{b}\left(0, T ; H^{s+1}(\Omega)\right)$ of the problem (6.34).

Furthermore, for small time interval $[0, T]$, there exists a constant $\varepsilon_{2}>0$ such that the following estimate holds whenever $\left\|\tilde{\mathbf{T}}^{n}\right\|_{C_{b}\left(0, T ; H^{s+1}\right)} \leq \varepsilon_{2}$ :

$$
\left\|\tilde{\mathbf{T}}^{n+1}\right\|_{C_{b}\left(0, T ; H^{s+1}\right)} \leq T\left(C_{2}\left\|\tilde{V}^{n+1}\right\|_{C_{b}\left(0, T ; H^{s+2}\right)}+C_{3}\right)
$$

where $C_{2}$ and $C_{3}$ are non-negative constants independent of $n$.

Lemma 6.5 Assume that $\tilde{q}^{n+1} \in C_{b}\left(0, T ; H^{s+1}(\Omega)\right)$ and $\tilde{V}^{n} \in C_{b}\left(0, T ; H^{s+2}(\Omega)\right)$ with $\operatorname{div} \tilde{V}^{n}=$ 0 in $\Omega \times[0, T], \tilde{V}^{n}=0$ on $\partial \Omega \times[0, T]$ and $\left\|\tilde{V}^{n}\right\|_{C_{b}\left(0, T ; H^{s+2}\right)} \leq \varepsilon_{1}$ for $\varepsilon_{1}>0$. Then there exists a unique $\tilde{p}^{n+1} \in C_{b}\left(0, T ; H^{s+1}(\Omega)\right)$, satisfying the problem (6.35).

Furthermore, the following estimate holds:

$$
\left\|\tilde{p}^{n+1}\right\|_{C_{b}\left(0, T ; H^{s+1}\right)} \leq T\left(C_{4}\left\|\tilde{q}^{n+1}\right\|_{C_{b}\left(0, T ; H^{s+1}\right)}+C_{5}\right) .
$$

The constants $C_{4}, C_{5}>0$ are independent of $n$.

The proofs of Lemmas 6.4 and 6.5 are similar to those in the small initial data case.

In the following lemma we will show that for small time interval $[0, \mathrm{~T}]$ the sequences $\tilde{V}^{n}, \tilde{q}^{n}, \tilde{p}^{n}$ and $\tilde{\mathbf{T}}^{n}$ are bounded independently of $n$ by small bounds.

Lemma 6.6 For a sufficiently small time interval $[0, T]$, there exists $\varepsilon$ small and $>0$ such that the sequences $\tilde{V}^{n}, \tilde{q}^{n}, \tilde{p}^{n}$ and $\tilde{\mathbf{T}}^{n}$ are bounded independently of $n$ by $\varepsilon$, respectively, in the spaces

$$
C_{b}\left(0, T ; H^{s+2}(\Omega)\right), C_{b}\left(0, T ; H^{s+1}(\Omega)\right), C_{b}\left(0, T ; H^{s+1}(\Omega)\right) \text { and } C_{b}\left(0, T ; H^{s+1}(\Omega)\right)
$$


Proof. Combining the estimates (6.36) and (6.37), we see that

$$
\left\|\tilde{V}^{n+1}\right\|_{C_{b}\left(0, \mathrm{~T} ; H^{s+2}\right)} \leq \mathrm{T} C_{1} C_{2}\left\|\tilde{V}^{n}\right\|_{C_{b}\left(0, \mathrm{~T} ; H^{s+2}\right)}+C_{1}\|\tilde{\mathbf{f}}\|_{C_{b}\left(0, \mathrm{~T} ; H^{s+1}\right)}+\mathrm{T} C_{1} C_{3} .
$$

Since $\tilde{\mathbf{f}}$ is assumed to be continuous and $\tilde{\mathbf{f}}(0)=0$, then $\|\tilde{\mathbf{f}}\|_{C_{b}\left(0, \mathrm{~T} ; H^{s+1}\right)}$ can be chosen small for $\mathrm{T}$ small. Hence, for $\mathrm{T}$ sufficiently small, there exists $\varepsilon>0$ so that

$$
C_{1}\|\tilde{\mathbf{f}}\|_{C_{b}\left(0, \mathrm{~T} ; H^{s+1}\right)}+\mathrm{T} C_{1} C_{3} \leq \frac{\varepsilon}{2} \text { and }{ }_{\mathrm{T}} C_{1} C_{2} \leq \frac{1}{2} .
$$

The estimate (6.39) becomes

$$
\left\|\tilde{V}^{n+1}\right\|_{C_{b}\left(0, \mathrm{~T} ; H^{s+2}\right)} \leq \frac{1}{2}\left\|\tilde{V}^{n}\right\|_{C_{b}\left(0, \mathrm{~T} ; H^{s+2}\right)}+\frac{\varepsilon}{2}
$$

Thus, by the principle of induction, we see that $\left\|\tilde{V}^{n}\right\|_{C_{b}\left(0, T ; H^{s+2}\right)} \leq \varepsilon$ for all $n \in \mathbb{N}$. Again, by combining the estimates (6.36), (6.37) and (6.38) we show that the other three sequences are bounded by $\varepsilon$.

\subsubsection{Convergence}

In this subsection, we prove that the sequences constructed above are convergent to a solution of the problem (1.12)-(1.16), and that this solution is unique.

Let us define the difference between two successive iterations by

$$
\left(\widetilde{\tilde{V}}^{n}, \widetilde{\tilde{q}}^{n}, \widetilde{\tilde{p}}^{n}, \tilde{\tilde{\mathbf{T}}}^{n}\right)=\left(\tilde{V}^{n}-\tilde{V}^{n-1}, \tilde{q}^{n}-\tilde{q}^{n-1}, \tilde{p}^{n}-\tilde{p}^{n-1}, \tilde{\mathbf{T}}^{n}-\tilde{\mathbf{T}}^{n-1}\right)
$$

We subtract the equations of the problems (6.33), (6.34) and (6.35) at iteration $n$ from those at iteration $n+1$. We see that the new sequences $\widetilde{\tilde{V}}^{n}, \widetilde{q}^{n}, \widetilde{\tilde{p}}^{n}$ and $\widetilde{\tilde{\mathbf{T}}}^{n}$ satisfy the following problems: 


$$
\begin{aligned}
& -\mu \Delta \tilde{\tilde{V}}^{n+1}-\operatorname{div}\left(g_{1}\left(\nabla \tilde{\tilde{V}}^{n+1}, \mathbf{T}_{0}\right)\right)+\operatorname{div}\left(\left(\tilde{\tilde{V}}^{n+1} \cdot \nabla\right) \mathbf{T}_{0}\right)+\nabla \widetilde{\tilde{q}}^{n+1} \\
& =\left(\nabla \widetilde{\tilde{V}}^{n}\right)^{\mathrm{T}}\left(\operatorname{div} \tilde{\mathbf{T}}^{n}-\tilde{\mathbf{f}}\right)+\left(\nabla\left(\tilde{V}^{n-1}+V^{0}\right)\right)^{\mathrm{T}} \operatorname{div} \tilde{\tilde{\mathbf{T}}}^{n}-\left(\widetilde{\tilde{V}}^{n} \cdot \nabla\right) \tilde{\mathbf{f}} \\
& -\sum_{j=1}^{3}\left[\left(\frac{\partial\left(\tilde{V}^{n}+V^{0}\right)}{\partial x_{j}} \cdot \nabla\right) \widetilde{\tilde{\mathbf{T}}}_{i j}^{n}+\left(\frac{\partial \tilde{\tilde{V}}^{n}}{\partial x_{j}} \cdot \nabla\right) \tilde{\mathbf{T}}_{i j}^{n-1}\right] \\
& +\operatorname{div}\left[g_{1}\left(\nabla \tilde{\tilde{V}}^{n}, \tilde{\mathbf{T}}^{n}\right)+g_{1}\left(\nabla\left(\tilde{V}^{n-1}+V^{0}\right), \tilde{\tilde{\mathbf{T}}}^{n}\right)\right] \\
& +\operatorname{div}\left[g_{2}\left(\tilde{\mathbf{T}}^{n}+\mathbf{T}_{0}\right)-g_{2}\left(\tilde{\mathbf{T}}^{n-1}+\mathbf{T}_{0}\right)\right] \quad \text { in } \Omega \times[0, \mathrm{~T}], \\
& \operatorname{div} \widetilde{\tilde{V}}^{n+1}=0 \quad \text { in } \Omega \times[0, \mathrm{~T}], \\
& \tilde{\tilde{V}}^{n+1}=0 \quad \text { on } \partial \Omega \times[0, \mathrm{~T}] .
\end{aligned}
$$

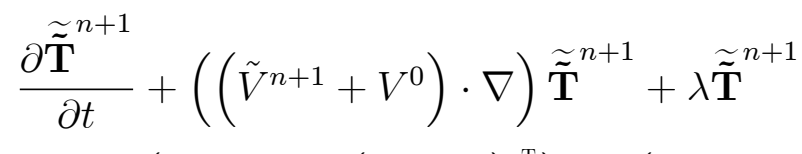

$$
\begin{aligned}
& =\mu\left(\nabla \tilde{\tilde{V}}^{n+1}+\left(\nabla \tilde{\tilde{V}}^{n+1}\right)^{\mathrm{T}}\right)-\left(\tilde{\tilde{V}}^{n+1} \cdot \nabla\right)\left(\tilde{\mathbf{T}}^{n}+\mathbf{T}_{0}\right) \\
& +g_{1}\left(\nabla \tilde{\tilde{V}}^{n+1}, \tilde{\mathbf{T}}^{n}+\mathbf{T}_{0}\right)+g_{1}\left(\nabla\left(\tilde{V}^{n}+V^{0}\right), \widetilde{\mathbf{T}}^{n}\right) \\
& +g_{2}\left(\tilde{\mathbf{T}}^{n}+\mathbf{T}_{0}\right)-g_{2}\left(\tilde{\mathbf{T}}^{n-1}+\mathbf{T}_{0}\right) \text { in } \Omega \times[0, \mathrm{~T}], \\
& \widetilde{\tilde{\mathbf{T}}}^{n+1}(\cdot, 0)=0 \quad \text { in } \Omega \text {. } \\
& \left.\begin{array}{l}
\frac{\partial \widetilde{\tilde{p}}^{n+1}}{\partial t}+\left(\left(\tilde{V}^{n}+V^{0}\right) \cdot \nabla\right) \widetilde{\tilde{p}}^{n+1}+\lambda \widetilde{\tilde{p}}^{n+1}=\widetilde{\tilde{q}}^{n+1}-\left(\tilde{\tilde{V}}^{n} \cdot \nabla\right) \tilde{p}^{n+1} \text { in } \Omega \times[0, \mathrm{~T}], \\
\widetilde{\tilde{p}}^{n+1}(\cdot, 0)=0 \text { in } \Omega .
\end{array}\right\}
\end{aligned}
$$

Now, by applying the estimates of Lemmas 6.3, 6.4 and 6.5, and by using the result of Lemma 6.6, we obtain the following estimates:

$$
\begin{array}{r}
\left\|\tilde{\tilde{V}}^{n+1}\right\|_{C_{b}\left(0, \mathrm{~T} ; H^{s+1}\right)}+\left\|\tilde{\tilde{q}}^{n+1}\right\|_{C_{b}\left(0, \mathrm{~T} ; H^{s}\right)} \leq K_{1}\left\|\widetilde{\tilde{\mathbf{T}}}^{n}\right\|_{C_{b}\left(0, \mathrm{~T} ; H^{s}\right)}+\varepsilon\left\|\widetilde{\tilde{V}}^{n}\right\|_{C_{b}\left(0, \mathrm{~T} ; H^{s+1}\right)}, \\
\left\|\tilde{\tilde{\mathbf{T}}}^{n+1}\right\|_{C_{b}\left(0, \mathrm{~T} ; H^{s}\right)} \leq \varepsilon\left(\left\|\widetilde{\tilde{V}}^{n+1}\right\|_{C_{b}\left(0, \mathrm{~T} ; H^{s+1}\right)}+\left\|\widetilde{\tilde{\mathbf{T}}}^{n}\right\|_{C_{b}\left(0, \mathrm{~T} ; H^{s}\right)}\right),
\end{array}
$$




$$
\left\|\widetilde{\tilde{p}}^{n+1}\right\|_{C_{b}\left(0, \mathrm{~T} ; H^{s}\right)} \leq \varepsilon\left(\left\|\widetilde{\tilde{q}}^{n+1}\right\|_{C_{b}\left(0, \mathrm{~T} ; H^{s}\right)}+\left\|\tilde{\tilde{V}}^{n}\right\|_{C_{b}\left(0, \mathrm{~T} ; H^{s+1}\right)}\right)
$$

In combining these estimates, we see that all the sequences $\left\|\widetilde{\tilde{p}}^{n}\right\|_{C_{b}\left(0, \mathrm{~T} ; H^{s}\right)},\|\widetilde{\tilde{q}}\|_{C_{b}\left(0, \mathrm{~T} ; H^{s}\right)}$, $\left\|\widetilde{\tilde{V}}^{n}\right\|_{C_{b}\left(0, \mathrm{~T} ; H^{s+1}\right)}$ and $\left\|\widetilde{\tilde{\mathbf{T}}}^{n}\right\|_{C_{b}\left(0, \mathrm{~T} ; H^{s}\right)}$ converge to zero. Then by the same argument as in Subsection 4.3 .2 , the sequences $\tilde{V}^{n}, \tilde{q}^{n}, \tilde{p}^{n}$ and $\tilde{\mathbf{T}}^{n}$ converge to a solution of the problems (6.30), (6.31) and (6.32) in the space

$$
\begin{aligned}
& C_{b}\left([0, \mathrm{~T}) ; H^{s+2}(\Omega)\right) \times C_{b}\left([0, \mathrm{~T}) ; H^{s+1}(\Omega) \cap L_{0}^{2}(\Omega)\right) \\
& \times C_{b}\left([0, \mathrm{~T}) ; H^{s+1}(\Omega) \cap L_{0}^{2}(\Omega)\right) \times C_{b}\left([0, \mathrm{~T}) ; H^{s+1}(\Omega)\right) .
\end{aligned}
$$

We prove the uniqueness in the same manner.

As a consequence of the above results we have the following theorem.

Theorem 6.7 Under the assumptions given in the beginning of this chapter and Section 6.1 with the condition that $\operatorname{div}\left(\mathbf{T}_{0}\right)-\mathbf{f}(\cdot, 0)$ is a gradient, there exists a unique local solution in time $(V, p, \mathbf{T})$ for the problem (1.12)-(1.16) in the following space:

$$
C_{b}\left([0, T) ; H^{s+2}(\Omega)\right) \times C_{b}\left([0, T) ; H^{s+1}(\Omega) \cap L_{0}^{2}(\Omega)\right) \times C_{b}\left([0, T) ; H^{s+1}(\Omega)\right) .
$$




\section{$\mathrm{C}^{\mathrm{h}^{\mathrm{aP}} \mathrm{e}_{\mathrm{r}}} 7$}

\section{Models of White-Metzner type in 3D flow with sufficiently small initial data}

\section{Contents}

7.1 Problem reformulation ................ 80

7.2 Assumptions on $\mu$ and $\lambda \ldots \ldots \ldots \ldots$. . . . . 81

7.3 Transformation to iterative scheme . . . . . . . . . . 82

7.4 Construction of the solution . . . . . . . . . . 83

$7.4 .1 \quad$ Existence . . . . . . . . . . . . . . . . . 83

7.4 .2 Convergence . . . . . . . . . . . . . . 86

In this chapter, we present well-posedness results for an unsteady flow problem of a quasistatic viscoelastic Maxwell fluid of White-Metzner type, in three-dimensional geometry, locally and globally in time, with small initial data.

Recall that White-Metzner models are described by the following constitutive equation, see Subsection 1.3.2:

$$
\frac{\partial \mathbf{T}}{\partial t}+(V \cdot \nabla) \mathbf{T}+\lambda\left(I_{D_{V}}\right) \mathbf{T}=\mu\left(\Pi_{D}\right)\left(\nabla V+(\nabla V)^{\mathrm{T}}\right)+g(\nabla V, \mathbf{T}),
$$


where, the scalar quantities $\lambda$ and $\mu$ depend on

$$
\Pi_{D_{V}} \equiv \frac{1}{2}\left|\frac{1}{2}\left(\nabla V+(\nabla V)^{\mathrm{T}}\right)\right|^{2} .
$$

The function $(M, N) \longmapsto g(M, N)$ is of class $C^{4}$ on $\mathbb{R}^{3 \times 3} \times \mathbb{R}^{3 \times 3}$ with $g$ and its gradient with respect to $(M, N)$ vanish at $(0,0)$. In this case the function $g$ satisfies the estimate

$$
\|g(\nabla V, \mathbf{T})\|_{H^{2}} \leq L\left(\|V\|_{H^{3}}^{2}+\|\mathbf{T}\|_{H^{2}}^{2}\right)
$$

for all $(V, \mathbf{T})$ in $H^{3}(\Omega) \times H^{2}(\Omega)$ with $\|V\|_{H^{3}},\|\mathbf{T}\|_{H^{2}} \leq c$. The constant $L=L(c)>0$.

The equations describing the flow problem for White-Metzner type models are:

$$
\begin{aligned}
& \operatorname{div} \mathbf{T}-\nabla p=\mathbf{f} \quad \text { in } \Omega \times[0, \mathrm{~T}), \mathrm{T} \in(0, \infty], \\
& \operatorname{div} V=0 \quad \text { in } \Omega \times[0, \mathrm{~T}), \\
& V=0 \quad \text { on } \partial \Omega \times[0, \mathrm{~T}), \\
& \frac{\partial \mathbf{T}}{\partial t}+(V \cdot \nabla) \mathbf{T}+\lambda\left(\Pi_{D_{V}}\right) \mathbf{T}=\mu\left(\Pi_{D_{V}}\right)\left(\nabla V+(\nabla V)^{\mathrm{T}}\right)+ \\
& g(\nabla V, \mathbf{T}) \text { in } \Omega \times[0, \mathrm{~T}), \\
& \mathbf{T}(\cdot, 0)=\mathbf{T}_{0} \quad \text { in } \Omega,
\end{aligned}
$$

with the constraint condition

$$
\operatorname{curl}\left(\operatorname{div}\left(\mathbf{T}_{0}\right)-\mathbf{f}(\cdot, 0)\right)=0
$$

The initial data $\mathbf{T}_{0}$ and the body force $\mathbf{f}$ that acting on the fluid are, respectively, functions in the spaces $H^{2}(\Omega)$ and $C_{b}\left([0, \mathrm{~T}) ; H^{2}(\Omega)\right) \cap C^{1}\left([0, \mathrm{~T}) ; H^{1}(\Omega)\right)$, and satisfy the estimate

$$
\left\|\mathbf{T}_{0}\right\|_{H^{s+1}}+\|\mathbf{f}\|_{C_{b}\left([0, T) ; H^{s+1}\right)}+\left\|\frac{\partial}{\partial t} \mathbf{f}\right\|_{C_{b}\left([0, T) ; H^{s}\right)} \leq \varepsilon,
$$

for some $\varepsilon>0$ and sufficiently small.

The main result is the following theorem.

Theorem 7.1 Let $T \in(0, \infty]$. Under the assumptions that will be imposed on $\mu$ and $\lambda$ in Section 7.2 (see page 81), the problem (7.2)-(7.6) will have a unique solution

$$
(\mathbf{T}, V, p) \in C_{b}\left([0, T) ; H^{2}(\Omega)\right) \times C_{b}\left([0, T) ; H^{3}(\Omega)\right) \times C_{b}\left([0, T) ; H^{2}(\Omega) \cap L_{0}^{2}(\Omega)\right) .
$$




\subsection{Problem reformulation}

By applying the divergence operator to both sides of the equation (7.5), we obtain

$$
\begin{aligned}
\frac{\partial}{\partial t} \operatorname{div} \mathbf{T}+ & (V \cdot \nabla) \operatorname{div} \mathbf{T}+\sum_{j=1}^{3}\left(\frac{\partial V}{\partial x_{j}} \cdot \nabla\right) \mathbf{T}_{i j}+\operatorname{div}\left(\lambda\left(\Pi_{D_{V}}\right) \mathbf{T}\right) \\
& =\operatorname{div}\left[\mu\left(\Pi_{D_{V}}\right)\left(\nabla V+(\nabla V)^{\mathrm{T}}\right)\right]+\operatorname{div}(g(\nabla V, \mathbf{T})) .
\end{aligned}
$$

We use the equation (7.2) to replace $\operatorname{div} \mathbf{T}$ by $\nabla p+\mathbf{f}$. In the equation obtained, we substitute $(V \cdot \nabla) \nabla p$ using the identity

$$
(V \cdot \nabla) \nabla p=\nabla((V \cdot \nabla) p)-(\nabla V)^{\mathrm{T}} \nabla p
$$

Thus, the equation (7.9) becomes

$$
\begin{aligned}
& -\operatorname{div}\left[\mu\left(\Pi_{D_{V}}\right)\left(\nabla V+(\nabla V)^{\mathrm{T}}\right)\right]+\nabla q=-\lambda(0) \mathbf{f}-\frac{\partial}{\partial t} \mathbf{f}-(V \cdot \nabla) \mathbf{f} \\
& \quad+(\nabla V)^{\mathrm{T}} \nabla p-\sum_{j=1}^{3}\left(\frac{\partial V}{\partial x_{j}} \cdot \nabla\right) \mathbf{T}_{i j}+\operatorname{div}\left[g(\nabla V, \mathbf{T})-\left(\lambda\left(\Pi_{D_{V}}\right)-\lambda(0)\right) \mathbf{T}\right],
\end{aligned}
$$

where

$$
q=\frac{\partial}{\partial t} p+(V \cdot \nabla) p+\lambda(0) p
$$

Note that $\mu$ and $\lambda$ are real valued functions defined on $[0, \infty)$. For simplicity in writing the equations, we will use the following notations:

$$
\left.\begin{array}{l}
\mu_{V}:=\mu\left(\Pi_{D_{V}}\right)-\mu(0), \mu_{0}:=\mu(0), \\
\lambda_{V}:=\lambda\left(\Pi_{D_{V}}\right)-\lambda(0), \lambda_{0}:=\lambda(0) .
\end{array}\right\}
$$

The problem (7.2)-(7.6) can be reformulated as follows: Find a vector field $V$, a tensor T, and two scalar functions $q$ and $p$ satisfying the following subproblems:

$$
\left.\begin{array}{ll}
\frac{\partial p}{\partial t}+(V \cdot \nabla) p+\lambda_{0} p=q & \text { in } \Omega \times[0, \mathrm{~T}), \\
p(\cdot, 0)=p_{0} & \text { in } \Omega,
\end{array}\right\}
$$




$$
\begin{array}{r}
-\operatorname{div}\left[\mu_{0}\left(\nabla V+(\nabla V)^{\mathrm{T}}\right)\right]+\nabla q=\operatorname{div}\left[g(\nabla V, \mathbf{T})-\lambda_{V} \mathbf{T}+\mu_{V}\left(\nabla V+(\nabla V)^{\mathrm{T}}\right)\right] \\
+(\nabla V)^{\mathrm{T}} \nabla p-\sum_{j=1}^{3}\left(\frac{\partial V}{\partial x_{j}} \cdot \nabla\right) \mathbf{T}_{i j}-\lambda_{0} \mathbf{f}-\frac{\partial}{\partial t} \mathbf{f}-(V \cdot \nabla) \mathbf{f} \text { in } \Omega \times[0, \mathrm{~T}),
\end{array}
$$

$\operatorname{div} V=0 \quad$ in $\Omega \times[0, \mathrm{~T})$,

$V=0 \quad$ on $\partial \Omega \times[0, \mathrm{~T})$,

$$
\begin{aligned}
& \frac{\partial \mathbf{T}}{\partial t}+(V \cdot \nabla) \mathbf{T}+\lambda_{0} \mathbf{T}=-\lambda_{V} \mathbf{T}+\left(\mu_{V}+\mu_{0}\right)\left(\nabla V+(\nabla V)^{\mathrm{T}}\right) \\
& +g(\nabla V, \mathbf{T}) \text { in } \Omega \times[0, \mathrm{~T}), \\
& \mathbf{T}(\cdot, 0)=\mathbf{T}_{0} \quad \text { in } \Omega .
\end{aligned}
$$

Note that $-\operatorname{div}\left[\mu_{0}\left(\nabla V+(\nabla V)^{\mathrm{T}}\right)\right]=-\mu_{0} \Delta V$ since $\operatorname{div} V=0$.

\subsection{Assumptions on $\mu$ and $\lambda$}

Here we assume that the real valued functions $\mu$ and $\lambda$ are $C^{3}([0, \infty))$, but analogous results can be obtained for the singular shear-thickening case where $\mu$ and $\lambda$ are $C^{3}(0, \infty)$ and satisfy the following conditions:

$$
\begin{aligned}
& |\mu(u)-\mu(0)| \leq c_{1} u^{\frac{r-2}{2}}, r>2, \quad c_{1}>0, \text { for all } u \geq 0, \\
& |\lambda(u)-\lambda(0)| \leq c_{2} u^{\frac{\omega-2}{2}}, \omega>2, \quad c_{2}>0, \text { for all } u \geq 0,
\end{aligned}
$$

Note that the fluid is called shear-thinning when $1<r<2[6]$.

For a class of viscoelastic fluids, the functions $\lambda\left(\Pi_{D_{V}}\right)$ and $\mu\left(\Pi_{D_{V}}\right)$ are given by the following equations, see Subsection 1.3.2:

$$
\left.\begin{array}{l}
\lambda\left(\Pi_{D_{V}}\right)=\lambda_{0}\left(1+a \Pi_{D_{V}}\right)^{\beta}, \\
\mu\left(I_{D_{V}}\right)=\mu_{0}\left[\left(1+a \Pi_{D_{V}}\right)^{\beta-\alpha}+b \Pi_{D_{V}}\left(1+a \Pi_{D_{V}}\right)^{-\beta-\alpha}\right],
\end{array}\right\}
$$

where $\lambda_{0}, \mu_{0}, a, b, \alpha$ and $\beta$ are constants characterizing the fluid. 
Proposition 7.2 Let $\mu$ and $\lambda$ be in $C^{3}([0, \infty))$. The functions $\mu_{V}$ and $\lambda_{V}$ defined on (7.12) map continuously $H^{3}(\Omega)$ into $H^{2}(\Omega)$, and satisfy the following estimates:

$$
\begin{aligned}
& \left\|\mu_{V}\right\|_{H^{2}} \leq C\|V\|_{H^{3}}, \\
& \left\|\lambda_{V}\right\|_{H^{2}} \leq C\|V\|_{H^{3}}
\end{aligned}
$$

for all $V \in H^{3}(\Omega)$ with $\|V\|_{H^{3}} \leq c$, and

$$
\begin{gathered}
\left\|\mu_{V}-\mu_{U}\right\|_{H^{1}} \leq C\|V-U\|_{H^{2}}, \\
\left\|\lambda_{V}-\lambda_{U}\right\|_{H^{1}} \leq C\|V-U\|_{H^{2}}
\end{gathered}
$$

for all $U, V \in H^{3}(\Omega)$ with $\|U\|_{H^{3}},\|V\|_{H^{3}} \leq c$, where $C=C(c)$ is a constant depending on $c$ and bounded if $c$ is small.

Proof. Use the chain rule formula for the composition of multivariable functions and Sobolev embedding theorem, see Subsection 2.2.5. To establish the estimates (7.19)-(7.22), one can use Taylor's formula with Lagrange's remainder in Banach spaces.

\subsection{Transformation to iterative scheme}

As in previous chapters, we prove the existence and uniqueness by transforming our problem to iterative linear subproblems. Then, we construct a sequence of solutions for those iterative subproblems, converging to a solution for our original problem.

We initialize the scheme by choosing

$$
\mathbf{T}^{0}=\mathbf{T}_{0}, V_{0}=0, p^{0}=p_{0}, q^{0}=q_{0}
$$


In subsequent iterations, we solve successively the following linear subproblems: The first one is a Stokes problem for $V^{n+1}$ and $q^{n+1}$

$$
\begin{array}{r}
-\mu_{0} \Delta V^{n+1}+\nabla q^{n+1}=\operatorname{div}\left[g\left(\nabla V^{n}, \mathbf{T}^{n}\right)-\lambda_{V^{n}} \mathbf{T}^{n}+\mu_{V^{n}}\left(\nabla V^{n}+\left(\nabla V^{n}\right)^{\mathrm{T}}\right)\right]+\left(\nabla V^{n}\right)^{\mathrm{T}} \nabla p^{n} \\
-\sum_{j=1}^{3}\left(\frac{\partial V^{n}}{\partial x_{j}} \cdot \nabla\right) \mathbf{T}_{i j}^{n}-\lambda_{0} \mathbf{f}-\frac{\partial}{\partial t} \mathbf{f}-\left(V^{n} \cdot \nabla\right) \mathbf{f} \text { in } \Omega \times[0, \mathrm{~T}),
\end{array}
$$

$\operatorname{div} V^{n+1}=0 \quad$ in $\Omega \times[0, \mathrm{~T})$,

$V^{n+1}=0 \quad$ on $\partial \Omega \times[0, \mathrm{~T})$.

The second, is an evolution problem for $\mathbf{T}^{n+1}$

$$
\begin{aligned}
& \frac{\partial \mathbf{T}^{n+1}}{\partial t}+\left(V^{n} \cdot \nabla\right) \mathbf{T}^{n+1}+\lambda_{0} \mathbf{T}^{n+1}=-\lambda_{V^{n}} \mathbf{T}^{n}+\mu_{V^{n}}\left(\nabla V^{n}+\left(\nabla V^{n}\right)^{\mathrm{T}}\right) \\
& +\mu_{0}\left(\nabla V^{n+1}+\left(\nabla V^{n+1}\right)^{\mathrm{T}}\right)+g\left(\nabla V^{n}, \mathbf{T}^{n}\right) \text { in } \Omega \times[0, \mathrm{~T}), \\
& \mathbf{T}^{n+1}(\cdot, 0)=\mathbf{T}_{0} \quad \text { in } \Omega,
\end{aligned}
$$

and another one for $p^{n+1}$

$$
\left.\begin{array}{ll}
\frac{\partial p^{n+1}}{\partial t}+\left(V^{n} \cdot \nabla\right) p^{n+1}+\lambda_{0} p^{n+1}=q^{n+1} & \text { in } \Omega \times[0, \mathrm{~T}), \\
p^{n+1}(\cdot, 0)=p_{0} & \text { in } \Omega .
\end{array}\right\}
$$

\subsection{Construction of the solution}

We prove in this section that the problem (7.2)-(7.6) has a unique solution, which can be obtained from the limit solution of iterative scheme (7.23)-(7.25).

\subsubsection{Existence}

In the following series of lemmas, we prove the existence and uniqueness of the solutions for the iterative subproblems (7.23)-(7.25). We also provide estimates that will be used to prove the convergence of the solutions sequence constructed. 
The first lemma concerning the result on Stokes problem (7.23).

Lemma 7.3 Let $T$ be in $(0, \infty]$. Let $\mathbf{f}$ be in the space $C_{b}\left([0, T) ; H^{2}(\Omega)\right) \cap C^{1}\left([0, T) ; H^{1}(\Omega)\right)$, $\mathbf{T}^{n} \in C_{b}\left([0, T) ; H^{2}(\Omega)\right)$ and $V^{n} \in C_{b}\left([0, T) ; H^{3}(\Omega)\right)$. The problem (7.23) has a unique solution

$$
\left(V^{n+1}, q^{n+1}\right) \in C_{b}\left([0, T) ; H^{3}(\Omega)\right) \times C_{b}\left([0, T) ; H^{2}(\Omega) \cap L_{0}^{2}(\Omega)\right) .
$$

Moreover, if $\left\|V^{n}\right\|_{C_{b}\left([0, T) ; H^{3}\right)} \leq \varepsilon_{1}$ and $\left\|\mathbf{T}^{n}\right\|_{C_{b}\left([0, T) ; H^{2}\right)} \leq \varepsilon_{2}$ for $\varepsilon_{1}, \varepsilon_{2}>0$, then we have the following estimate:

$$
\left\|V^{n+1}\right\|_{C_{b}\left([0, T) ; H^{3}\right)}+\left\|q^{n+1}\right\|_{C_{b}\left([0, T) ; H^{2}\right)} \leq C_{1}\left(\left\|V^{n}\right\|_{H^{3}}^{2}+\left\|p^{n}\right\|_{H^{2}}^{2}+\left\|\mathbf{T}^{n}\right\|_{H^{2}}^{2}+\varepsilon\right)
$$

where $C_{1}$ is a non-negative constant independent of $n$, and $\varepsilon$ is a bound of the norms of $\mathbf{f}$ and $\frac{\partial}{\partial t} \mathbf{f}$.

Proof. By Proposition 3.1, the problem (7.23) has a unique solution in the above spaces and satisfies the estimate

$$
\left\|V^{n+1}\right\|_{H^{3}}+\left\|q^{n+1}\right\|_{H^{2}} \leq C\left\|\mathcal{M}\left(V^{n}, p^{n}, \mathbf{T}^{n}, \mathbf{f}\right)\right\|_{H^{1}},
$$

where $\mathcal{M}\left(V^{n}, p^{n}, \mathbf{T}^{n}, \mathbf{f}\right)$ denotes the right hand side of the first equation of (7.23).

By Sobolev embedding theorem and the estimate (7.1), and using the inequality $2 a b \leq a^{2}+b^{2}$, we obtain

$$
\left\|\mathcal{M}\left(V^{n}, p^{n}, \mathbf{T}^{n}, \mathbf{f}\right)\right\|_{H^{1}} \leq C\left(\left\|V^{n}\right\|_{H^{3}}^{2}+\left\|p^{n}\right\|_{H^{2}}^{2}+\left\|\mathbf{T}^{n}\right\|_{H^{2}}^{2}+\left\|\lambda_{V^{n}}\right\|_{H^{2}}^{2}+\left\|\mu_{V^{n}}\right\|_{H^{2}}^{2}+\varepsilon\right)
$$

Using the inequalities (7.19), (7.20), and taking the supremum over $t$, we obtain the inequality required.

The next two lemmas are devoted to results on the evolutions problems (7.24) and (7.25).

Lemma 7.4 Let $T \in(0, \infty]$. Assume that $\mathbf{T}^{n} \in C_{b}\left([0, T) ; H^{2}(\Omega)\right)$ and $V^{n}, V^{n+1}$ are in the space $C_{b}\left([0, T) ; H^{3}(\Omega)\right)$ with $\operatorname{div} V^{n}(t)=0$ in $\Omega, V^{n}(t)=0$ on $\partial \Omega$ and $\left\|V^{n}\right\|_{C_{b}\left([0, T) ; H^{3}\right)} \leq \varepsilon_{1}$ for a sufficiently small $\varepsilon_{1}>0$. Then there exists a unique solution $\mathbf{T}^{n+1} \in C_{b}\left([0, T) ; H^{2}(\Omega)\right)$ 
of the problem (7.24). Moreover, if $\left\|\mathbf{T}^{n}\right\|_{C_{b}\left([0, T) ; H^{s+1}\right)} \leq \varepsilon_{2}$ for $\varepsilon_{2}>0$, then the following estimate holds:

$$
\begin{aligned}
& \left\|\mathbf{T}^{n+1}\right\|_{C_{b}\left([0, T) ; H^{2}\right)} \leq \\
& \quad C_{2}\left(\left\|\mathbf{T}_{0}\right\|_{H^{2}}+\left\|V^{n+1}\right\|_{C_{b}\left([0, T) ; H^{3}\right)}+\left\|V^{n}\right\|_{C_{b}\left([0, T) ; H^{3}\right)}^{2}+\left\|\mathbf{T}^{n}\right\|_{C_{b}\left([0, T) ; H^{2}\right)}^{2}\right),
\end{aligned}
$$

where $C_{2}$ is a non-negative constant independent of $n$.

Lemma 7.5 Let $T \in(0, \infty]$. Assume that $q^{n+1} \in C_{b}\left([0, T) ; H^{2}(\Omega)\right)$ and $V^{n}$ be in the space $C_{b}\left([0, T) ; H^{3}(\Omega)\right)$ with $\operatorname{div} V^{n}=0$ in $\Omega \times[0, T), V^{n}=0$ on $\partial \Omega \times[0, T)$ and $\left\|V^{n}\right\|_{C_{b}\left([0, T) ; H^{3}\right)} \leq$ $\varepsilon_{1}$ for $\varepsilon_{1}$ sufficiently small and $>0$. Then there exists a unique $p^{n+1} \in C_{b}\left([0, T) ; H^{2}(\Omega)\right)$, satisfying the problem (7.25). Furthermore, the following estimate holds:

$$
\left\|p^{n+1}\right\|_{C_{b}\left([0, T) ; H^{2}\right)} \leq C_{3}\left(\left\|p_{0}\right\|_{H^{2}}+\left\|q^{n+1}\right\|_{C_{b}\left([0, T) ; H^{2}\right)}\right)
$$

where the constant $C_{3}$ is non-negative and independent of $n$.

The proofs of Lemmas 7.4 and 7.5 are similar to those in the small initial data case with $\mu$ and $\lambda$ constants, see Section 4.3.

In the subsequent lemma we show that for sufficiently small initial value $\mathbf{T}_{0}$ and body force $\mathbf{f}$, the sequences $V^{n}, q^{n}, p^{n}$ and $\mathbf{T}^{n}$ are bounded independently of $n$ by small bounds.

Lemma 7.6 Let $T \in(0, \infty]$. Let $\varepsilon$ be sufficiently small and $\varepsilon>0$ for which

$$
\left\|\mathbf{T}_{0}\right\|_{H^{2}}+\|\mathbf{f}\|_{C_{b}\left([0, T) ; H^{2}\right)}+\left\|\frac{\partial}{\partial t} \mathbf{f}\right\|_{C_{b}\left([0, T) ; H^{1}\right)} \leq \varepsilon .
$$

In this case $\left\|p_{0}\right\|_{H^{2}}$ is also $\leq \varepsilon$ and there exists $C>0$ independent of $n$ such that the sequences $V^{n}, q^{n}, p^{n}$ and $\mathbf{T}^{n}$ are bounded independently of $n$ by $C \varepsilon$, respectively, in the spaces

$$
C_{b}\left([0, T) ; H^{3}(\Omega)\right), C_{b}\left([0, T) ; H^{2}(\Omega)\right), C_{b}\left([0, T) ; H^{2}(\Omega)\right) \text { and } C_{b}\left([0, T) ; H^{2}(\Omega)\right)
$$

Proof. Let $\alpha_{n}, \beta_{n}, \gamma_{n}$ and $\lambda_{n}$ be bounds at iteration $n$ for $\left\|V^{n}\right\|_{C_{b}\left([0, T) ; H^{3}\right)},\left\|q^{n}\right\|_{C_{b}\left([0, \mathrm{~T}) ; H^{2}\right)}$, $\left\|p^{n}\right\|_{C_{b}\left([0, \mathrm{~T}) ; H^{2}\right)}$ and $\left\|\mathbf{T}^{n}\right\|_{C_{b}\left([0, \mathrm{~T}) ; H^{2}\right)}$, respectively. Let $\sigma_{n}=\alpha_{n}+\gamma_{n}+\lambda_{n}$. 
From the estimates (7.26), (7.28) and (7.29), we see that

$$
\begin{aligned}
& \alpha_{n+1}+\beta_{n+1} \leq C_{1}\left(\alpha_{n}^{2}+\gamma_{n}^{2}+\lambda_{n}^{2}+\varepsilon\right), \\
& \lambda_{n+1} \leq C_{2}\left(\varepsilon+\alpha_{n+1}+\alpha_{n}^{2}+\lambda_{n}^{2}\right), \\
& \gamma_{n+1} \leq C_{3}\left(\varepsilon+\beta_{n+1}\right),
\end{aligned}
$$

where $C_{1}, C_{2}$ and $C_{3}$ are non-negative constants and independent of $n$.

Multiplying the inequality (7.30) by $1+C_{2}$ and (7.32) by $\frac{1+C_{2}}{C_{3}}$, and then adding them together to $(7.31)$, leads to

$$
\sigma_{n+1} \leq a \varepsilon+b \sigma_{n}^{2}
$$

where

$$
a=\frac{1+2 C_{2}+C_{1}+C_{1} C_{2}}{\min \left\{1, \frac{1+C_{2}}{C_{3}}\right\}}, \quad b=\frac{C_{1}\left(1+C_{2}\right)+C_{2}}{\min \left\{1, \frac{1+C_{2}}{C_{3}}\right\}} .
$$

Thus, by the principle of induction, we see that $\alpha_{n}, \beta_{n}, \gamma_{n}$ and $\lambda_{n}$ remain bounded independently of $n$ by $C \varepsilon$ if $\varepsilon$ chosen sufficiently small.

\subsubsection{Convergence}

We shall prove that the sequences constructed above are convergent in appropriate spaces to a solution of the problem (7.2)-(7.6), and that this solution is unique.

Let us define the difference between two successive iterations by

$$
\left(\tilde{V}^{n}, \tilde{q}^{n}, \tilde{p}^{n}, \tilde{\mathbf{T}}^{n}\right)=\left(V^{n}-V^{n-1}, q^{n}-q^{n-1}, p^{n}-p^{n-1}, \mathbf{T}^{n}-\mathbf{T}^{n-1}\right) .
$$


We subtract the equations of the problems (7.23), (7.24) and (7.25) at iteration $n$ from those at iteration $n+1$. We see that the new sequences satisfy the following problems:

$$
\begin{aligned}
& -\mu_{0} \Delta \tilde{V}^{n+1}+\nabla \tilde{q}^{n+1}=\operatorname{div}\left[g\left(\nabla V^{n}, \mathbf{T}^{n}\right)-g\left(\nabla V^{n-1}, \mathbf{T}^{n-1}\right)\right] \\
& -\operatorname{div}\left[\lambda_{V^{n}} \tilde{\mathbf{T}}^{n}+\left(\lambda_{V^{n}}-\lambda_{V^{n-1}}\right) \mathbf{T}^{n-1}\right]+\left(\nabla V^{n}\right)^{\mathrm{T}} \nabla \tilde{p}^{n}+\left(\nabla \tilde{V}^{n}\right)^{\mathrm{T}} \nabla p^{n-1} \\
& +\operatorname{div}\left[\mu_{V^{n}}\left(\nabla \tilde{V}^{n}+\left(\nabla \tilde{V}^{n}\right)^{\mathrm{T}}\right)+\left(\mu_{V^{n}}-\mu_{V^{n-1}}\right)\left(\nabla V^{n-1}+\left(\nabla V^{n-1}\right)^{\mathrm{T}}\right)\right] \\
& -\sum_{j=1}^{3}\left(\frac{\partial V^{n}}{\partial x_{j}} \cdot \nabla\right) \tilde{\mathbf{T}}_{i j}^{n}-\sum_{j=1}^{3}\left(\frac{\partial \tilde{V}^{n}}{\partial x_{j}} \cdot \nabla\right) \mathbf{T}_{i j}^{n-1}-\left(\tilde{V}^{n} \cdot \nabla\right) \mathbf{f} \text { in } \Omega \times[0, \mathrm{~T}), \\
& \operatorname{div} \tilde{V}^{n+1}=0 \quad \text { in } \Omega \times[0, \mathrm{~T}), \\
& \tilde{V}^{n+1}=0 \quad \text { on } \partial \Omega \times[0, \mathrm{~T}), \\
& \begin{aligned}
\frac{\partial \tilde{\mathbf{T}}^{n+1}}{\partial t} & +\left(V^{n} \cdot \nabla\right) \tilde{\mathbf{T}}^{n+1}+\lambda_{0} \tilde{\mathbf{T}}^{n+1}=-\left(\tilde{V}^{n} \cdot \nabla\right) \mathbf{T}^{n}-\lambda_{V^{n}} \tilde{\mathbf{T}}^{n}-\left(\lambda_{V^{n}}-\lambda_{V^{n-1}}\right) \mathbf{T}^{n-1} \\
& +\mu_{V^{n}}\left(\nabla \tilde{V}^{n}+\left(\nabla \tilde{V}^{n}\right)^{\mathrm{T}}\right)+\left(\mu_{V^{n}}-\mu_{V^{n-1}}\right)\left(\nabla V^{n-1}+\left(\nabla V^{n-1}\right)^{\mathrm{T}}\right) \\
& +\mu_{0}\left(\nabla \tilde{V}^{n+1}+\left(\nabla \tilde{V}^{n+1}\right)^{\mathrm{T}}\right)+g\left(\nabla V^{n}, \mathbf{T}^{n}\right)-g\left(\nabla V^{n-1}, \mathbf{T}^{n-1}\right) \text { in } \Omega \times[0, \mathrm{~T})
\end{aligned} \\
& \tilde{\mathbf{T}}^{n+1}(\cdot, 0)=0 \quad \text { in } \Omega, \\
& \left.\begin{array}{ll}
\frac{\partial \tilde{p}^{n+1}}{\partial t}+\left(V^{n} \cdot \nabla\right) \tilde{p}^{n+1}+\lambda_{0} \tilde{p}^{n+1}=\tilde{q}^{n+1}-\left(\tilde{V}^{n} \cdot \nabla\right) p^{n} & \text { in } \Omega \times[0, \mathrm{~T}), \\
\tilde{p}^{n+1}(\cdot, 0)=0 & \text { in } \Omega .
\end{array}\right\}
\end{aligned}
$$

Let $\mathrm{T} \in(0, \infty]$. Let $\varepsilon$ be a bound of the sequences $V^{n}, q^{n}, p^{n}$ and $\mathbf{T}^{n}$, respectively, in the spaces

$$
C_{b}\left([0, \mathrm{~T}) ; H^{3}(\Omega)\right), C_{b}\left([0, \mathrm{~T}) ; H^{2}(\Omega)\right), C_{b}\left([0, \mathrm{~T}) ; H^{2}(\Omega)\right) \text { and } C_{b}\left([0, \mathrm{~T}) ; H^{2}(\Omega)\right)
$$

In addition, we assume that

$$
\left\|\mathbf{T}_{0}\right\|_{H^{2}}+\|\mathbf{f}\|_{C_{b}\left([0, T) ; H^{2}\right)}+\left\|\frac{\partial}{\partial t} \mathbf{f}\right\|_{C_{b}\left([0, T) ; H^{1}\right)} \leq \varepsilon .
$$


The new sequences $\tilde{V}^{n}, \tilde{q}^{n}, \tilde{p}^{n}$ and $\tilde{\mathbf{T}}^{n}$ satisfy the following estimates:

$$
\begin{gathered}
\left\|\tilde{V}^{n+1}\right\|_{C_{b}\left([0, \mathrm{~T}) ; H^{2}\right)}+\left\|\tilde{q}^{n+1}\right\|_{C_{b}\left([0, \mathrm{~T}) ; H^{1}\right)} \leq \\
K_{1} \varepsilon\left(\left\|\tilde{V}^{n}\right\|_{C_{b}\left([0, \mathrm{~T}) ; H^{2}\right)}+\left\|\tilde{\mathbf{T}}^{n}\right\|_{C_{b}\left([0, \mathrm{~T}) ; H^{1}\right)}+\left\|\tilde{p}^{n}\right\|_{C_{b}\left([0, \mathrm{~T}) ; H^{1}\right)}\right) \\
\left\|\tilde{\mathbf{T}}^{n+1}\right\|_{C_{b}\left([0, \mathrm{~T}) ; H^{1}\right)} \leq \\
K_{2}\left(\varepsilon\left\|\tilde{\mathbf{T}}^{n}\right\|_{C_{b}\left([0, \mathrm{~T}) ; H^{1}\right)}+\varepsilon\left\|\tilde{V}^{n}\right\|_{C_{b}\left([0, \mathrm{~T}) ; H^{2}\right)}+\left\|\tilde{V}^{n+1}\right\|_{C_{b}\left([0, \mathrm{~T}) ; H^{2}\right)}\right) \\
\left\|\tilde{p}^{n+1}\right\|_{C_{b}\left([0, \mathrm{~T}) ; H^{1}\right)} \leq K_{3}\left(\varepsilon\left\|\tilde{V}^{n}\right\|_{C_{b}\left([0, \mathrm{~T}) ; H^{2}\right)}+\left\|\tilde{q}^{n+1}\right\|_{C_{b}\left([0, \mathrm{~T}) ; H^{1}\right)}\right)
\end{gathered}
$$

where $K_{1}, K_{2}$ and $K_{3}$ are non-negative constants independent of $n$. By the same argument as in the case where $\mu$ and $\lambda$ are constants, see Subsection 4.3.2, the sequences $V^{n}, p^{n}$ and $\mathbf{T}^{n}$ converge to the solution of the problem (7.2)-(7.6) in the space space

$$
C_{b}\left([0, \mathrm{~T}) ; H^{3}(\Omega)\right) \times C_{b}\left([0, \mathrm{~T}) ; H^{2}(\Omega) \cap L_{0}^{2}(\Omega)\right) \times C_{b}\left([0, \mathrm{~T}) ; H^{2}(\Omega)\right)
$$




\section{Conclusion and perspectives}

A formal solution to the problem describing an unsteady flow of a quasistatic viscoelastic Maxwell fluid can be constructed by iterative methods.

The convergence is guaranteed in the following cases:

- Three-dimensional flow, in a bounded domain, locally and globally in time for sufficiently small initial data.

- Simple shear flow, i.e. a spatially one-dimensional problem, locally in time with arbitrary initial data.

- Some three-dimensional flow problems, in a bounded domain, locally in time with large initial data.

- Models of White-Metzner type in three-dimensional flow with sufficiently small initial data.

The convergence of the formal solution in three-dimensional flow problems with large initial data depends on the unique solvability of a second order PDEs boundary value problem. The existence, possibly lack of existence, and uniqueness of solutions for this problem in the cases left untreated here, remain a challenge for future work. I think that an example of non-uniqueness can be constructed by using non-elementary functions. 


\section{Bibliography}

[1] R. Adams, Sobolev Spaces, Academic Press, New York, San Francisco, London, 1975. $19,22,23,24,39$

[2] S. Agmon, A. Douglis, and L. Nirenberg, Estimates near the boundary for solutions of elliptic partial differential equations satisfying general boundary conditions II, Communications on pure and applied mathematics, Vol. 17, 1964, p. 35-92.

[3] I. Babuška and I. Hlaváček, On the existence and uniqueness of solutions in the theory of viscoelasticity, Arch. Mech. Stos. 18 (1966), 47-83. 3

[4] H. Beirão da Veiga, Boundary-value problems for a class of first order partial differential equations in Sobolev spaces and applications to the Euler flow, Rend. Sem. Math. Univ. Padova 79 (1988), 247-273. 33

[5] G. M. Constantine, T. H. Savits, A Multivariate Faà di Bruno Formula with Applications, Trans. Amer. Math. Soc. 348 (1996), 503-520. 25

[6] C. Ebmeyer, Regularity in Sobolev spaces of steady flows of fluids with sheardependent viscosity, Math. Methods Appl. Sci. 29 (2006), 1687-1707. 81

[7] R. E. Gaidos, and R. Darby, Numerical simulation and change in type in the developing flow of a nonlinear viscoelastic fluid, J. of Non-Newtonian Fluid Mech. 29 (1988) 59-79. 14 
[8] E. Guyon, J.-P. Hulin , L. Petit, C. D. Mitescu, Physical Hydrodynamics, Oxford University Press, USA, 2001. 2, 6, 8, 11

[9] V. Girault, P. A. Raviart, Finite Elements Methods for Navier-Stokes Equations, Springer Series in Computational Mathematics, vol. 5, Springer, Berlin, New York, Tokyo 1986. 22, 23, 29

[10] C. Guillopé and J.C. Saut, Global existence and one-dimensional nonlinear stability of shearing motions of viscoelastic fluids of Oldroyd type, RAIRO Modél. Math. Anal. Numér. 24 (1990), 369-401. 2

[11] C. Guillopé and J.C. Saut, Existence results for the flow of viscoelastic fluids with a differential constitutive law, Nonlin. Anal. TMA 15 (1990), 849-869. 2

[12] A. Hakim, Existence of slow steady flows of viscoelastic fluids of White-Metzner type. Comput. Math. Appl. 38 (1999), no. 11-12, 239-247. 13

[13] A. Hakim, Existence results for the flow of viscoelastic fluids of White-Metzner type. Extracta Math. 9 (1994), no. 1, 51-55.

[14] A. Hakim, Mathematical analysis of viscoelastic fluids of White-Metzner type. J. Math. Anal. Appl. 185 (1994), no. 3, 675-705. 14

[15] R. R. Huilgol and N. Phan-Thien, Fluid Mechanics of Viscoelasticity, General Principles, Constitutive Modelling, Analytical and Numerical Techniques, Elsevier, Amsterdam, 1997. 6, 8

[16] D. D. Joseph, Fluid Dynamics of Viscoelastic Liquids, Springer-Verlag, New York, 1990. 1

[17] T. Kato, Linear Evolution Equations of "hyperbolic" type, J. Fac. Sci. Univ. Tokyo, Sect. I, 17 (1970), 241-258. 32

[18] K. Kobayasi, On a Theorem for Linear Evolution Equations of Hyperbolic Type, J. Math. Soc. Japan, 31 (1979), 647-654. 
[19] O. A. Ladyzhenskaya, The Mathematical Theory of Viscous Incompressible Flow, Gordon and Breach, New York, 1969. 29

[20] R. G. Larson, Constitutive Equations for Polymer Melts and Solutions, Butterworts Series in Chemical Engineering, Boston 1987. 13

[21] C. W. Macosko, Rheology: Principles, Measurements and Applications, VCH publishers Inc, New York USA, 1994. 1, 10, 11, 12, 13

[22] A. Y. Malkin, Rheology fundamentals, ChemTec Publishing, 1994. 10

[23] L. Molinet and R. Talhouk, On the global and periodic regular flows of viscoelastic fluids with a differential constitutive law, Nonlin. Diff. Eq. Appl. 11 (2004), 349-359. 2

[24] A. Pazy, Semigroups of Linear Operators and Applications to Partial differential Equations, Applied Math. Sciences, Vol. 44, Springer-Verlag, New York, 1983. 30

[25] N. Phan-Thien, Understanding Viscoelasticity, Basics of Rheology, Springer-Verlag Berlin Heidelberg, 2002. 1, 6, 8, 11

[26] M. Renardy, Existence of Slow Steady Flows of Viscoelastic Fluids with Differential Constitutive Equations, Z. angew. Math. Mech. 65 (1985), 449-451. 2, 38, 40, 70

[27] M. Renardy, W. J. Hrusaand J. A. Nohel, Mathematical Problems in Viscoelasticity, Pitman Monographs and Surveys in Pure and Applied Mathematics, Longman, Harlow, 1987. 2, 40

[28] M. Renardy, Local existence of solutions of the Dirichlet initial-boundary value problem for incompressible hypoelastic materials, SIAM J. Math. Anal. 21 (1990), 1369-1385. 2

[29] M. Renardy, Mathematical Analysis of Viscoelastic Flows, CBMS-NSF Regional Conference Series in Applied Mathematics 73, SIAM, Philadelphia, 2000. 1, 2, 11, 13, 38, 40

[30] M. Renardy, R. C. Rogers, An Introduction to Partial Differential Equations, Second edition, (Text in Applied Mathematics, Vol 13), Springer-Verlag, New York, 2004. 19, $22,23,27$ 
[31] J. H. Spurk, Fluid mechanics, Springer-Verlag Berlin New York, 1997. 10

[32] R. Temam, Navier-Stokes Equations: Theory and Numerical Analysis, North-HollandAmsterdam, New York, Oxford, 1984. 29, 70

[33] C. Verdier and D. D. Joseph, Change of type and loss of evolution of the WhiteMetzner model, J. of Non-Newtonian Fluid Mech. 31 (1989), no. 3, 325-343. 13

[34] F. F. Wu, C. A. Desoer, Global Inverse Function Theorem, IEEE Trans. Circuit Theory CT-19 (1972), 199-201. 27

[35] E. Zeidler, Nonlinear Functional Analysis and Its Applications, II/A, Linear Monotone Operators, Springer, New York, 1990. 24, 39 